\title{
A Boundary Layer Theory for Diffusively Perturbed Transport Around a Heteroclinic Cycle
}

\author{
RICHARD B. SOWERS \\ University of Illinois at Urbana-Champaign
}

\begin{abstract}
We consider a two-dimensional transport equation subject to small diffusive perturbations. The transport equation is given by a Hamiltonian flow near a compact and connected heteroclinic cycle. We investigate approximately harmonic functions corresponding to the generator of the perturbed transport equation. In particular, we investigate such functions in the boundary layer near the heteroclinic cycle; the space of these functions gives information about the likelihood of a particle moving a mesoscopic distance into one of the regions where the transport equation corresponds to periodic oscillations (i.e., a "well" of the Hamiltonian). We find that we can construct such approximately harmonic functions (which can be used as "corrector functions" in certain averaging questions) when certain macroscopic "gluing conditions" are satisfied. This provides a different perspective on some previous work of Freidlin and Wentzell on stochastic averaging of Hamiltonian systems. (c) 2004 Wiley Periodicals, Inc.
\end{abstract}

\section{Contents}

1. Introduction 1

2. The Setup 2

3. Approximations 11

4. Calculations and Estimates 21

5. Correctors $\quad 35$

6. Proof of Main Result $\quad 40$

Appendix A. Global Aspects of $\mathfrak{z} \quad 40$

Appendix B. Khasminskii PDE $\quad 48$

$\begin{array}{ll}\text { Bibliography } & 54\end{array}$

\section{Introduction}

In a recent paper [9] we developed a PDE-based analysis (using the notion of perturbed test functions) of certain gluing asymptotics found in the study of diffusively perturbed conservative dynamical systems. We considered stochastic averaging for a small random perturbation of a certain Hamiltonian system that 
modeled the behavior of a Newtonian mass in a double-well potential. The existence of the two wells implied that the Hamiltonian system had a homoclinic orbit. Except on this homoclinic orbit, standard stochastic averaging techniques could be applied as the strength of the random perturbation tended to 0 . At the homoclinic orbit, gluing conditions allowed us to complete the averaged picture.

These gluing conditions were understood by Freidlin and Wentzell [6] and Freidlin and Weber [4, 5] (see also Neřshtadt [7]); primarily, they defined a sort of "coin flip" that governed the dynamics at the homoclinic orbit. Our work of [9] represented a slight generalization of their results via a significantly different method.

The analysis of [9] relied upon solving a certain singular perturbations problem that involved several elliptic-parabolic PDEs coupled by boundary data. Our goal here is to develop this PDE analysis in its own right. This PDE result should be understood as a framework for understanding general gluing conditions.

\section{The Setup}

Let's start with a two-dimensional $C^{\infty}$-manifold $\mathfrak{M}$ with symplectic form $\omega$. Fix a function $\mathrm{H} \in C^{\infty}(\mathfrak{M})$ and let $\bar{\nabla} \mathrm{H}$ be the symplectic gradient of $\mathrm{H}$ (i.e., $\bar{\nabla} \mathrm{H}$ is the unique vector field such that $\omega(X, \nabla \mathrm{H})=X \mathrm{H}$ for all vector fields $X)$.

ASSUMPTION 2.1 We assume that the set $\Gamma \stackrel{\text { def }}{=} \mathrm{H}^{-1}(0)$ is a compact and connected subset of $\mathfrak{M}$ and that the set $\mathscr{X} \stackrel{\text { def }}{=}\{x \in \mathfrak{M}: d \mathrm{H}(x)=0\}$ of critical points of $\mathrm{H}$ is contained in $\Gamma$ and that $\mathrm{H}$ is nondegenerate (i.e., $D^{2} \mathrm{H}$ is nonsingular) at all points of $\mathscr{X}$. We assume that $\mathrm{H}$ has at least one critical point.

These assumptions imply that that $\mathscr{X}$ is finite.

Consider now the transport equation $\bar{\nabla} \mathrm{H} u=0$, which tells us how a particle is moved around by $\bar{\nabla} \mathrm{H}$. Of course, this PDE is satisfied by any function of $\mathrm{H}$; we want to regularize this PDE near $\boldsymbol{\Gamma}$.

AsSUMPTION 2.2 Let $(\cdot, \cdot)$ be a Riemannian metric on $\mathfrak{M}$ that is related to $\omega$; i.e., there is a smooth fiber map $\mathrm{J}: T \mathfrak{M} \rightarrow T \mathfrak{M}$ such that $\left.\mathrm{J}\right|_{T_{x} \mathfrak{M}}$ is an isometry (with respect to $(\cdot, \cdot)$ ) for each $x \in \mathfrak{M}$ such that $\omega(X, Y)=(X, \mathrm{~J} Y)$ for all vector fields $X$ and $Y$. Let $\Delta$ and $\nabla$, respectively, be the Laplace-Beltrami and gradient operators defined by $(\cdot, \cdot)$. As usual, we define $\|X\| \stackrel{\text { def }}{=} \sqrt{(X, X)}$ for all $X \in T \mathfrak{M}$. Let $\mathbf{b}$ be a vector field on $\mathfrak{M}$ and let $\beth \in C^{2}(\mathfrak{M})$ be such that $\inf _{x \in \mathfrak{M}} \beth(x)>0$.

Define the operator

$$
\mathscr{L} \stackrel{\text { def }}{=} \frac{1}{2} \beth \Delta+\mathbf{b} .
$$

For each $\varepsilon>0$, consider the PDE

$$
\left(\bar{\nabla} \mathrm{H}, \nabla u^{\varepsilon}\right)+\varepsilon^{2} \mathscr{L} u^{\varepsilon}=0 .
$$

Physically, this PDE describes the dynamics of a particle following the flow of $\bar{\nabla} \mathrm{H}$ but subject to small diffusive perturbations. 
Remark 2.3. Our construction is fairly general. If the Riemannian metric and symplectic form are not related, then (since the vector space of 2 -forms on $\mathbb{R}^{2}$ is onedimensional), we can premultiply the symplectic gradient by a scalar-valued function and, by appropriately incorporating this function into I, reduce it to the above problem.

For each $\hbar>0$, let $\Gamma(\hbar)$ be the connected component of $\{x \in \mathfrak{M}:|\mathrm{H}(x)|<\hbar\}$ that contains $\boldsymbol{\Gamma}$; since $\mathfrak{M}$ is locally compact (it is a manifold) and $\boldsymbol{\Gamma}$ is by assumption compact, there is an $\hbar \in(0,1)$ such that $\overline{\Gamma(\hbar)}$ is a compact subset of $\mathfrak{M}^{1}{ }^{1}$

DEFINITION 2.4 Define $M \stackrel{\text { def }}{=} \boldsymbol{\Gamma}(\hbar)$.

Define next the flow

$$
\begin{aligned}
& \dot{\mathfrak{z}}_{t}(x)=\bar{\nabla} \mathrm{H}\left(\mathfrak{z}_{t}(x)\right), t \in \mathbb{R}, \quad x \in \mathrm{M}, \\
& \mathfrak{z}_{0}(x)=x,
\end{aligned}
$$

of diffeomorphisms of $\mathrm{M}$. This flow generates the characteristics of the dominant part of the PDE (2.1). For each $x \in M$, define

$$
\mathfrak{T}(x) \stackrel{\text { def }}{=} \inf \left\{t>0: \mathfrak{z}_{t}(x)=x\right\} \quad(\text { inf } \varnothing \stackrel{\text { def }}{=} \infty)
$$

LEMMA 2.5 We have that $\mathfrak{T}=\infty$ on $\boldsymbol{\Gamma}$ and $\mathfrak{T}<\infty$ on $\mathrm{M} \backslash \boldsymbol{\Gamma}$.

In other words, $\mathfrak{z}$ has no periodic orbits on $\boldsymbol{\Gamma}$, and all orbits on $\mathrm{M} \backslash \boldsymbol{\Gamma}$ are periodic. We will give the proof of this result in Appendix A.

We want to understand a bit about the solution space of the PDE (2.1). Our interest in the solution space is particularly focused upon boundary data. Recall that the solution space of a harmonic operator on a bounded domain is intimately related to the relative likelihoods that an associated diffusion exits the domain through different parts of the boundary of the domain. In lieu of a fixed boundary here, we are interested in how the associated diffusion exits a mesoscopic region around $\boldsymbol{\Gamma}$. Our assumptions imply that $\mathrm{M} \backslash \boldsymbol{\Gamma}$ has a finite collection $\left\{\mathrm{M}_{\ell}: \ell \in \Lambda\right\}$ of connected components, all of which are open subsets of $M$, and all of which are invariant under $\mathfrak{z}$; here $\Lambda$ is simply the index set; see Figure 2.1. Heuristically, we are interested in the asymptotic effect of diffusion between the different $\mathrm{M}_{\ell}$ 's as $\varepsilon \searrow 0$. If a particle starts in one of the $\mathrm{M}_{\ell}$ 's, it will rotate (with speed of $O(1)$ ) around the closed orbits of $\mathfrak{z}$ in $M_{\ell}$ and slowly diffuse across these orbits, eventually reaching a region near $\boldsymbol{\Gamma}$ (in time $1 / \varepsilon^{2}$ ). Although it is in this neighborhood of $\boldsymbol{\Gamma}$, it will visit all of the $\mathrm{M}_{\ell}$ 's. Sooner or later, it will move a mesoscopic distance away from $\Gamma$ into one of the $M_{\ell}$ 's. The relative likelihood of making a mesoscopic excursion into the different $\mathrm{M}_{\ell}$ 's is given by gluing conditions.

We can also look at this phenomenon from the standpoint of singular perturbations. Away from $\Gamma, \bar{\nabla} \mathrm{H}$ is nonzero, so the operator $\bar{\nabla} \mathrm{H}+\varepsilon^{2} \mathscr{L}$ is, to first order,

\footnotetext{
${ }^{1}$ Cover $\boldsymbol{\Gamma}$ by neighborhoods in $\mathfrak{M}$ whose closures are compact. Extract a finite subcover; this will give an open set $\mathcal{O}$ containing $\boldsymbol{\Gamma}$ whose closure is compact. Since the compact set $\boldsymbol{\Gamma}$ and the closed set $\mathfrak{M} \backslash \mathcal{O}$ are disjoint, $|\mathrm{H}|$ has a nonzero minimum on $\mathfrak{M} \backslash \mathcal{O}$.
} 


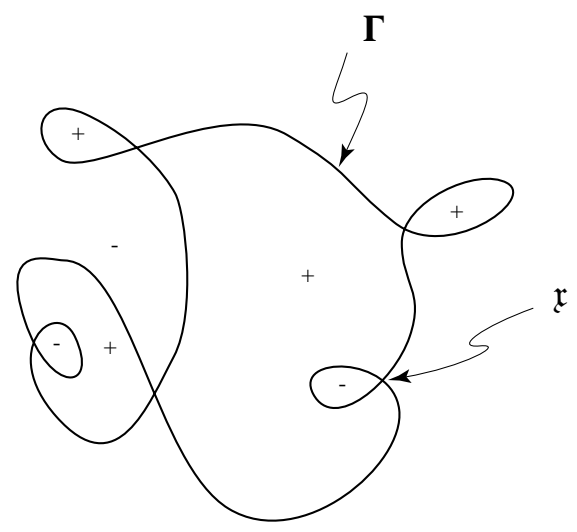

FIGURE 2.1. Heteroclinic cycle. "十" indicates a region in $\Lambda_{P}$; “-” indicates a region in $\Lambda_{W}$.

the vector field $\bar{\nabla} \mathrm{H}$. Standard methods allow us to construct outer expansions of the PDE (2.1) in the $\mathrm{M}_{\ell}$ 's. We then need an inner expansion in a boundary layer near $\boldsymbol{\Gamma}$. In this boundary layer, the second-order operator $\mathscr{L}$ enforces smoothness between the different inner expansions. Understood in this way, we can think of a different inner expansion in each of the $\mathrm{M}_{\ell}$ 's; starting from an outer expansion, we want to match up these inner expansions on $\boldsymbol{\Gamma}$. Since the fast drift is of order 1 and the slow diffusion is of order $\varepsilon^{2}$, we want the second-order effects of the slow diffusion to equilibrate with the fast drift. This means that the boundary layer must be of order $\mathrm{H}=O(\varepsilon)$.

Our main result is an analysis of a rescaled form of the PDE (2.1). For each $\varepsilon \in(0,1)$, define the operator

$$
\mathscr{L}^{\varepsilon} \stackrel{\text { def }}{=} \frac{1}{\varepsilon^{2}} \bar{\nabla} \mathrm{H}+\mathscr{L}
$$

and consider now the PDE $\mathscr{L}^{\varepsilon} u^{\varepsilon}=0$; in this rescaled PDE, we are considering a particle that, except at $\boldsymbol{\Gamma}$, has closed orbits of period of order $\varepsilon^{2}$, and transversal diffusion of order 1 across these orbits. Our main result is an inner expansion in the boundary layer near $\boldsymbol{\Gamma}$. For every $\ell \in \Lambda$, define $\Gamma_{\ell} \stackrel{\text { def }}{=} \boldsymbol{\Gamma} \cap \partial \mathrm{M}_{\ell}$ (i.e., $\Gamma_{\ell}$ is the part of $\Gamma$ that borders $\mathrm{M}_{\ell}$ ) and define a gluing coefficient

$$
\mathscr{G}_{\ell} \stackrel{\text { def }}{=} \int_{z \in \Gamma_{\ell}} I(z)\|\nabla \mathrm{H}(z)\| \mathscr{H}^{1}(d z),
$$

where $\mathscr{H}^{1}$ is the one-dimensional Hausdorff measure generated by $(\cdot, \cdot)$. Define next subsets $\Lambda_{P}$ and $\Lambda_{W}$ of $\Lambda$ (corresponding to "peaks" and "wells") as

$$
\Lambda_{P} \stackrel{\text { def }}{=}\left\{\ell \in \Lambda: \mathrm{H}>0 \text { on } \mathrm{M}_{\ell}\right\} \quad \text { and } \quad \Lambda_{W} \stackrel{\text { def }}{=}\left\{\ell \in \Lambda: \mathrm{H}<0 \text { on } \mathrm{M}_{\ell}\right\} .
$$


Next fix a vector $\left(\mathrm{v}_{\ell}: \ell \in \Lambda\right)$ of real numbers such that

$$
\sum_{\ell \in \Lambda_{P}} \mathrm{v}_{\ell} \mathscr{G}_{\ell}=\sum_{\ell \in \Lambda_{W}} \mathrm{v}_{\ell} \mathscr{G}_{\ell}
$$

(these are the gluing conditions) and define

$$
\digamma(x) \stackrel{\text { def }}{=} \sum_{\ell \in \Lambda} \mathrm{v}_{\ell} \mathrm{H}(x) \chi_{\mathrm{M}_{\ell}}(x), \quad x \in \mathrm{M} .
$$

Note that $\digamma$ is continuous on $\mathrm{M}$ but that its derivatives in general fail to exist at $\Gamma$ (unless, for example, all of the $v_{\ell}$ 's are identical). Our main result is a corrector for this discontinuity of derivatives.

THEOREM 2.6 There are two sequences $\left(\Phi^{\varepsilon,+}: \varepsilon \in(0,1)\right)$ and $\left(\Phi^{\varepsilon,-}: \varepsilon \in(0,1)\right)$ of elements of $C(\mathrm{M}) \cap C^{2}(\mathrm{M} \backslash \boldsymbol{\Gamma})$ such that $\digamma+\Phi^{\varepsilon,+}$ and $\digamma+\Phi^{\varepsilon,-}$ are both in $C^{1}(\mathrm{M}) \cap C^{2}(\mathrm{M} \backslash \Gamma)$ for all $\varepsilon \in(0,1)$ and such that

$$
\begin{gathered}
\varlimsup_{\varepsilon \searrow 0} \sup _{x \in \mathrm{M}}\left|\Phi^{\varepsilon, \pm}(x)\right|=0, \\
\varlimsup_{\varepsilon \searrow 0} \inf _{x \in \mathrm{M}}\left(\mathscr{L}^{\varepsilon} \Phi^{\varepsilon,+}\right)(x) \geq 0, \quad \text { and } \quad \varlimsup_{\varepsilon \searrow 0} \sup _{x \in \mathrm{M}}\left(\mathscr{L}^{\varepsilon} \Phi^{\varepsilon,-}\right)(x) \leq 0 .
\end{gathered}
$$

The proof is finalized in Section 6. The point of this is that we can asymptotically correct (in the sense of super- and subsolutions of $\mathscr{L}^{\varepsilon} u^{\varepsilon} \equiv 0$ ) for the nonsmoothness (i.e., loss of derivative) in $\digamma$ via small functions. The import of this is as follows: We can construct outer expansions of (2.1) in each $M_{\ell}$ subject to appropriate boundary conditions away from $\boldsymbol{\Gamma}$ and subject to continuity at $\boldsymbol{\Gamma}$. The dominant part of these outer expansions will be functions only of $H$, and the transversal (to $\boldsymbol{\Gamma})$ derivatives of these outer expansions may fail to agree at $\boldsymbol{\Gamma}$. We can characterize this loss of differentiability by an appropriate $\digamma$. Theorem 2.6 tells us that we can correct for this loss of derivative in the outer expansions if the derivatives obey the gluing relations of (2.2). In other words, we are assured that we can asymptotically solve (2.1) when the far-field boundary conditions imply gluable discontinuities at $\boldsymbol{\Gamma}$.

Note that Theorem 2.6 tells us that we can construct a corrector if the gluing conditions are satisfied. We cannot at the moment prove the converse, that if the gluing conditions are not satisfied, then we cannot construct such a corrector (although the solvability criterion for the functional analysis of Appendix B probably contains some parts of the proof of such a converse).

Remark 2.7. We can also add a zeroth-order operator to $\mathscr{L}$ and get the same result (using the same $\Phi^{\varepsilon, \pm}$ 's).

We also note that these corrector functions are exactly what is needed to construct perturbed test functions for proving the limit theorems of [6] (see [10]). 


\subsection{Local Behavior near the $\mathfrak{x}$ 's}

Since we will be carrying out a number of calculations near the $\mathfrak{x}$ 's, let's develop a common picture near those points.

Let $\mathbf{0} \stackrel{\text { def }}{=}(0,0)$ be the origin of $\mathbb{R}^{2}$. Define $\tilde{\mathrm{H}}\left(x_{1}, x_{2}\right) \stackrel{\text { def }}{=} x_{1} x_{2}$ for all $\left(x_{1}, x_{2}\right) \in \mathbb{R}^{2}$.

Lemma 2.8 Fix $\mathfrak{x} \in \mathscr{X}$. There is an open neighborhood $\mathscr{U}_{\mathfrak{x}}$ (contained in $\mathfrak{M}$ ) of $\mathfrak{x}$ and an orientation-preserving map $\phi_{\mathfrak{x}}: \mathscr{U}_{\mathfrak{x}} \rightarrow \mathbb{R}^{2}$ such that $\phi_{\mathfrak{x}}(\mathfrak{x})=\mathbf{0}, \phi_{\mathfrak{x}}$ is a diffeomorphism from $\mathscr{U}_{\mathfrak{x}}$ to $\phi_{\mathfrak{x}}\left(\mathscr{U}_{\mathfrak{x}}\right)$, and $\mathrm{H}=\tilde{\mathrm{H}} \circ \phi_{\mathfrak{x}}$ on $\mathscr{U}_{\mathfrak{x}}$.

PROOF: Fix $\mathfrak{x} \in \mathscr{X}$. By the Morse lemma, there is an open neighborhood $\mathscr{U}_{\mathfrak{x}}$ of $\mathfrak{x}$ and a chart $\check{\phi}_{\mathfrak{x}}: \mathscr{U}_{\mathfrak{x}} \rightarrow \mathbb{R}^{2}$ such that $\check{\phi}_{\mathfrak{x}}(\mathfrak{x})=\mathbf{0}$ and such that $\mathrm{H} \check{\phi}_{\mathfrak{x}}^{-1}\left(x_{1}, x_{2}\right)=$ $x_{1}^{2}-x_{2}^{2}$ for all $\left(x_{1}, x_{2}\right) \in \check{\phi}_{\mathfrak{x}}\left(\mathscr{U}_{\mathfrak{x}}\right)$. Define then

$$
\iota\left(x_{1}, x_{2}\right) \stackrel{\text { def }}{=} \begin{cases}\frac{1}{2}\left(x_{1}+x_{2}, x_{2}-x_{1}\right) & \text { if } \check{\phi}_{\mathfrak{x}} \text { is positively oriented, } \\ \frac{1}{2}\left(x_{1}+x_{2}, x_{1}-x_{2}\right) & \text { if } \check{\phi}_{\mathfrak{x}} \text { is negatively oriented }\end{cases}
$$

for all $\left(x_{1}, x_{2}\right) \in \mathbb{R}^{2}$. Define $\phi_{\mathfrak{x}} \stackrel{\text { def }}{=} \iota^{-1} \check{\phi}_{\mathfrak{x}}$ on $\mathscr{U}_{\mathfrak{x}}$.

For convenience, define $\tilde{\phi}_{\mathfrak{x}} \stackrel{\text { def }}{=} \phi_{\mathfrak{x}}^{-1}$ on $\phi_{\mathfrak{x}}\left(\mathscr{U}_{\mathfrak{x}}\right)$.

Define $\left\|\left(x_{1}, x_{2}\right)\right\|_{\infty} \stackrel{\text { def }}{=} \max \left\{\left|x_{1}\right|,\left|x_{2}\right|\right\}$ for all $\left(x_{1}, x_{2}\right) \in \mathbb{R}^{2}$.

DEFINITION 2.9 Let $\check{\varpi} \in(0,1)$ be small enough that the set $\check{\square} \stackrel{\text { def }}{=}\left\{x \in \mathbb{R}^{2}\right.$ : $\left.\|x\|_{\infty}<\check{\varpi}\right\}$ is contained in $\bigcap_{\mathfrak{x} \in \mathscr{X}} \phi_{\mathfrak{x}}\left(\mathscr{U}_{\mathfrak{x}}\right)$.

Then $\tilde{\phi}_{\mathfrak{x}}(\breve{\square})$ is the neighborhood where we can start our local analysis near $\mathfrak{x}$.

Let's next push various things through the $\phi_{\mathfrak{x}}$ 's, using Euclidean geometry on $\mathbb{R}^{2}$ as a reference. Let $(\cdot, \cdot)_{E}$ be the standard Euclidean metric on $T \mathbb{R}^{2}$, let $\nabla_{E}$ be the standard Euclidean gradient operator, let $\omega_{E} \stackrel{\text { def }}{=} d x_{1} \wedge d x_{2}$ be the standard symplectic form on $T^{*} \mathbb{R}^{2}$, and let $\bar{\nabla}_{E}$ be the standard Euclidean symplectic gradient operator; then

$$
\left(\left(\bar{\nabla}_{E} \tilde{\mathrm{H}}\right) f\right)\left(x_{1}, x_{2}\right)=x_{1} \frac{\partial f}{\partial x_{1}}\left(x_{1}, x_{2}\right)-x_{2} \frac{\partial f}{\partial x_{2}}\left(x_{1}, x_{2}\right)
$$

for all $\left(x_{1}, x_{2}\right) \in \mathbb{R}^{2}$ and all $f \in C^{1}\left(\mathbb{R}^{2}\right)$. Next fix $\mathfrak{x} \in \mathscr{X}$. Define the second-order operators $\tilde{\mathscr{L}}_{\mathfrak{x}}$ and $\tilde{\mathscr{L}}_{\mathfrak{x}}^{\varepsilon}$ on $C^{2}(\check{\square})$ and the function $\tilde{\mathcal{I}}_{\mathfrak{x}} \in C^{\infty}(\check{\square})$ by

$$
\begin{array}{rlrl}
\left(\tilde{\mathscr{L}}_{\mathfrak{x}} f\right)(x) & \stackrel{\text { def }}{=}\left(\mathscr{L}\left(f \circ \phi_{\mathfrak{x}}\right)\right)\left(\tilde{\phi}_{\mathfrak{x}}(x)\right), & & x \in \check{\square}, \\
\left(\tilde{\mathscr{L}}_{\mathfrak{x}}^{\varepsilon} f\right)(x) & \stackrel{\text { def }}{=}\left(\mathscr{L}^{\varepsilon}\left(f \circ \phi_{\mathfrak{x}}\right)\right)\left(\tilde{\phi}_{\mathfrak{x}}(x)\right), & \\
\tilde{\beth}_{\mathfrak{x}}(x) & \stackrel{\text { def }}{=} \beth\left(\tilde{\phi}_{\mathfrak{x}}(x)\right), & x \in \check{\square} .
\end{array}
$$


Let's also define a gradient associated with $\tilde{\mathscr{L}}_{\mathfrak{x}}$. Define

$$
\tilde{g}_{\mathfrak{x}, i, j}(x) \stackrel{\text { def }}{=}\left(D \phi_{\mathfrak{x}}\left(\left.\frac{\partial}{\partial x_{i}}\right|_{x}\right), D \phi_{\mathfrak{x}}\left(\left.\frac{\partial}{\partial x_{j}}\right|_{x}\right)\right)
$$

for all $x \in \check{\square}$, let $\tilde{g}_{\mathfrak{x}}^{-1}(x)$ be the matrix inverse of $\left(\tilde{g}_{\mathfrak{x}, i, j}(x): i, j \in\{1,2\}\right)$, and let $\tilde{g}^{-1 / 2}(x)$ be the (symmetric) square root of $\tilde{g}^{-1}(x)$. For any $f \in C^{1}(\check{\square})$, define then

$$
\left.\tilde{\nabla}_{\mathfrak{x}} f(x) \stackrel{\text { def }}{=} \sum_{j \in\{1,2\}} \tilde{g}_{\mathfrak{x}, i, j}^{-1 / 2}(x) \frac{\partial f}{\partial x_{i}}(x) \frac{\partial}{\partial x_{j}}\right|_{x}
$$

for all $x \in \check{\square}$. If $f \in C^{2}(\check{\square})$ and $\Phi \in C^{2}(\mathbb{R})$, then

$$
\begin{gathered}
\left\|\nabla\left(f \circ \phi_{\mathfrak{x}}\right)\left(\tilde{\phi}_{\mathfrak{x}}(x)\right)\right\|^{2}=\left\|\tilde{\nabla}_{\mathfrak{x}} f(x)\right\|_{E}^{2}, \\
\left(\tilde{\mathscr{L}}_{\mathfrak{x}}(\Phi \circ f)\right)(x)=\dot{\Phi}(f(x))\left(\tilde{\mathscr{L}}_{\mathfrak{x}} f\right)(x)+\frac{1}{2} \ddot{\Phi}(f(x))\left\|\tilde{\nabla}_{\mathfrak{x}} f(x)\right\|_{E}^{2},
\end{gathered}
$$

for all $x \in \square$. Pushing $\omega$ through $\phi_{\mathfrak{x}}$, let's also define

$$
\tilde{\mathrm{B}}_{\mathfrak{x}} \stackrel{\text { def }}{=} \frac{d\left(\tilde{\phi}_{\mathfrak{x}}^{*} \omega\right)}{d \omega_{E}} \quad \text { on } \check{\square}
$$

in the usual way; ${ }^{2}$ see $\left[1\right.$, sec. 7.33.C]. Since the chart $\phi_{\mathfrak{x}}$ is orientation preserving, $\tilde{\mathrm{B}}_{\mathfrak{x}}>0$ on $\check{\square}$. For any $x \in \check{\square}$ and any vector $X \in T_{x} \mathbb{R}^{2}$, then

$$
\begin{aligned}
\tilde{\mathrm{B}}_{\mathfrak{x}}(x) \omega_{E}\left(D \phi_{\mathfrak{x}} \bar{\nabla} \mathrm{H}\left(\tilde{\phi}_{\mathfrak{x}}(x)\right), X\right) & =\tilde{\phi}_{\mathfrak{x}}^{*} \omega\left(D \phi_{\mathfrak{x}} \bar{\nabla} \mathrm{H}\left(\tilde{\phi}_{\mathfrak{x}}(x)\right), X\right) \\
& =\omega\left(\bar{\nabla} \mathrm{H}\left(\tilde{\phi}_{\mathfrak{x}}(x)\right), D \tilde{\phi}_{\mathfrak{x}} X\right) \\
& =\left(D \tilde{\phi}_{\mathfrak{x}} X\right) \mathrm{H} \\
& =X\left(\mathrm{H} \circ \tilde{\phi}_{\mathfrak{x}}\right)=X \tilde{\mathrm{H}}=\omega_{E}\left(\bar{\nabla}_{E} \tilde{\mathrm{H}}, X\right)_{E},
\end{aligned}
$$

so

$$
D \phi_{\mathfrak{x}} \bar{\nabla} \mathrm{H}\left(\tilde{\phi}_{\mathfrak{x}}(x)\right)=\frac{1}{\tilde{\mathrm{B}}_{\mathfrak{x}}(x)} \bar{\nabla}_{E} \tilde{\mathrm{H}}(x) .
$$

Hence, for each $\mathfrak{x} \in \mathscr{X}$,

$$
\left(\tilde{\mathscr{L}}_{\mathfrak{x}}^{\varepsilon} f\right)(x)=\frac{1}{\varepsilon^{2} \tilde{\mathfrak{B}}_{\mathfrak{x}}(x)}\left(\bar{\nabla}_{E} \tilde{\mathrm{H}}, \nabla_{E} f\right)_{E}(x)+\left(\tilde{\mathscr{L}}_{\mathfrak{x}} f\right)(x)
$$

for all $x \in \check{\square}, \varepsilon \in(0,1)$, and $f \in C^{2}(\check{\square})$.

\footnotetext{
${ }^{2}$ In other words, $\omega\left(D \tilde{\phi}_{\mathfrak{x}}(X), D \tilde{\phi}_{\mathfrak{x}}(Y)\right)=\tilde{\mathrm{B}}_{\mathfrak{x}}(x) \omega_{E}(X, Y)$ for any $x \in \breve{\square}$ and any $X$ and $Y$ in $T_{x} \mathbb{R}^{2}$.
} 


\subsection{Boundary Coordinates}

Let's now define some boundary coordinates on the $\Gamma_{\ell}$ 's. We need a coordinate that measures position transversal to $\boldsymbol{\Gamma}$ and one that measures position along $\boldsymbol{\Gamma}$. We naturally let $\mathrm{H}$ measure position transversal to $\Gamma$. We define a collection of Khasminskii coordinates to measure position along $\boldsymbol{\Gamma}$.

LEMMA 2.10 There is $a \bar{\Theta} \in C^{\infty}(\boldsymbol{\Gamma} \backslash \mathscr{X})$ such that

$$
(\bar{\nabla} \mathrm{H}, \nabla \bar{\Theta})=\beth\|\nabla \mathrm{H}\|^{2}
$$

on $\boldsymbol{\Gamma} \backslash \mathscr{X}$. Furthermore, $\bar{\Theta}$ is uniformly continuous on $\boldsymbol{\Gamma} \backslash \mathscr{X}$.

The proof will be given in Appendix A. Our motivation for this PDE will be given below (or see the discussion of Section 8.1 of [9]).

Next, let's define something like a "covering map" from $\mathbb{R}$ to each $\Gamma_{\ell}$. Define

$$
\mathcal{I}_{\ell} \stackrel{\text { def }}{=}\left[0, \mathscr{G}_{\ell}\right), \quad \ell \in \Lambda \text {. }
$$

Lemma 2.11 Fix $\ell \in \Lambda$. There is a map $\mathbf{p}_{\ell}: \mathbb{R} \rightarrow \Gamma_{\ell}$ such that

(i) $\mathbf{p}_{\ell}(0) \in \mathscr{X}$,

(ii) $\mathbf{p}_{\ell}$ is a surjection,

(iii) for each $x \in \mathrm{M}_{\ell}$, the map $\mathbf{p}_{\ell}: \mathbb{R} \rightarrow \mathrm{M}_{\ell} \cup \Gamma_{\ell}$ is homotopic to the map $t \mapsto \mathfrak{z}_{t}(x)$ from $\mathbb{R}$ to $\mathrm{M}_{\ell} \cup \Gamma_{\ell}$, and

(iv) $\mathbf{p}_{\ell}$ is $\mathscr{G}_{\ell}$-periodic.

Defining $A_{\ell} \stackrel{\text { def }}{=} \mathbf{p}_{\ell}^{-1}\left(\Gamma_{\ell} \backslash \mathscr{X}\right)$, we also have that

(v) $\mathbb{R} \backslash A_{\ell}$ is discrete,

(vi) $\mathbf{p}_{\ell}$ is $C^{\infty}$ on $A_{\ell} \stackrel{\text { def }}{=} \mathbf{p}_{\ell}^{-1}\left(\Gamma_{\ell} \backslash \mathscr{X}\right)$, and

$$
\dot{\mathbf{p}}_{\ell}(t)=\frac{\bar{\nabla} \mathrm{H}}{\mathrm{J}\|\bar{\nabla} \mathrm{H}\|^{2}}\left(\mathbf{p}_{\ell}(t)\right)
$$

for all $t \in A_{\ell}$, and

(vii) $\left.\mathbf{p}_{\ell}\right|_{A_{\ell} \cap \mathcal{I}_{\ell}}$ is injective.

We will prove this in Appendix A.

LEMMA 2.12 For each $\ell \in \Lambda$, there is a locally constant function $\overline{\mathrm{C}}_{\ell}: \Gamma_{\ell} \backslash \mathscr{X} \rightarrow$ $\mathbb{R}$ such that $\bar{\Theta}\left(\mathbf{p}_{\ell}(t)\right)+\overline{\mathbf{C}}_{\ell}\left(\mathbf{p}_{\ell}(t)\right)=$ t for all $t \in A_{\ell} \cap \mathcal{I}_{\ell}$.

We will also prove this result in Appendix A.

Remark 2.13. Thus $\left.\mathbf{p}_{\ell}\right|_{A_{\ell} \cap \mathcal{I}_{\ell}}$ is a diffeomorphism from $A_{\ell} \cap \mathcal{I}_{\ell}$ to $\Gamma_{\ell} \backslash \mathscr{X}$ with inverse $\bar{\Theta}+\overline{\mathbf{C}}_{\ell}$.

Let's understand the behavior of $\bar{\Theta}$ near $\mathscr{X}$ 's. Define

$$
\tilde{\boldsymbol{\Gamma}} \stackrel{\text { def }}{=}\{x \in \check{\square}: \tilde{\mathrm{H}}(x)=0\} \text {. }
$$


Fix $\mathfrak{x} \in \mathscr{X}$. Set

$$
\bar{\Theta}_{\mathfrak{x}}^{\sim}(x) \stackrel{\text { def }}{=} \bar{\Theta}\left(\tilde{\phi}_{\mathfrak{x}}(x)\right)-\lim _{t \searrow 0} \bar{\Theta}\left(\tilde{\phi}_{\mathfrak{x}}(t x)\right)
$$

for all $x \in \tilde{\boldsymbol{\Gamma}} \backslash\{\boldsymbol{0}\}$ (the uniform continuity result of Lemma 2.10 implies that the limit exists). Define

$$
\begin{aligned}
\tilde{F}_{\mathfrak{x}}\left(x_{1}, x_{2}\right) \stackrel{\text { def }}{=} \tilde{\beth}_{\mathfrak{x}}\left(x_{1}, x_{2}\right) \tilde{\mathrm{B}}_{\mathfrak{x}}\left(x_{1}, x_{2}\right) \| & \nabla_{\mathfrak{x}} \tilde{\mathrm{H}}\left(x_{1}, x_{2}\right) \|_{E}^{2} \\
= & \tilde{\beth}_{\mathfrak{x}}\left(x_{1}, x_{2}\right) \tilde{\mathrm{B}}_{\mathfrak{x}}\left(x_{1}, x_{2}\right)\left\{\tilde{g}_{\mathfrak{x}, 1,1}^{-1}\left(x_{1}, x_{2}\right) x_{2}^{2}\right. \\
& \left.+2 \tilde{g}_{\mathfrak{x}, 1,2}^{-1}\left(x_{1}, x_{2}\right) x_{1} x_{2}+\tilde{g}_{\mathfrak{x}, 2,2}^{-1}\left(x_{1}, x_{2}\right) x_{1}^{2}\right\}
\end{aligned}
$$

for all $\left(x_{1}, x_{2}\right) \in \check{\square}$; then $\bar{\Theta}_{\mathfrak{x}}^{\sim}$ satisfies the PDE

$$
\begin{aligned}
\left(\bar{\nabla}_{E} \tilde{\mathrm{H}}, \nabla_{E} \bar{\Theta}_{\mathfrak{x}}^{\sim}\right)_{E} & =\tilde{F}_{\mathfrak{x}} \quad \text { on } \tilde{\boldsymbol{\Gamma}} \backslash\{\mathbf{0}\}, \\
& \lim _{\substack{x \rightarrow \mathbf{0} \\
x \in \tilde{\Gamma} \backslash\{\mathbf{0}\}}} \bar{\Theta}_{\mathfrak{x}}^{\sim}(x)=0 .
\end{aligned}
$$

Define

$$
\begin{aligned}
& \tilde{f}_{\mathfrak{x}}^{V}\left(x_{2}\right) \stackrel{\text { def }}{=} \frac{\tilde{F}_{\mathfrak{x}}\left(0, x_{2}\right)}{x_{2}^{2}}=\tilde{\beth}_{\mathfrak{x}}\left(0, x_{2}\right) \tilde{\mathrm{B}}_{\mathfrak{x}}\left(0, x_{2}\right) \tilde{g}_{\mathfrak{x}, 1,1}^{-1}\left(0, x_{2}\right), \\
& \tilde{f}_{\mathfrak{x}}^{H}\left(x_{1}\right) \stackrel{\text { def }}{=} \frac{\tilde{F}_{\mathfrak{x}}\left(x_{1}, 0\right)}{x_{1}^{2}}=\tilde{\beth}_{\mathfrak{x}}\left(x_{1}, 0\right) \tilde{\mathrm{B}}_{\mathfrak{x}}\left(x_{1}, 0\right) \tilde{g}_{\mathfrak{x}, 2,2}^{-1}\left(x_{1}, 0\right),
\end{aligned}
$$

for all $x_{1}$ and $x_{2}$ in $I_{1}^{*} \stackrel{\text { def }}{=}(-\check{\varpi}, \check{\varpi})$. We thus have that

$$
x_{1} \frac{\partial \tilde{\Theta}_{\mathfrak{x}}}{\partial x_{1}}\left(x_{1}, 0\right)=x_{1}^{2} \tilde{f}_{\mathfrak{x}}^{H}\left(x_{1}\right) \quad \text { and } \quad-x_{2} \frac{\partial \tilde{\Theta}_{\mathfrak{x}}}{\partial x_{2}}\left(0, x_{2}\right)=x_{2}^{2} \tilde{f}_{\mathfrak{x}}^{V}\left(x_{2}\right)
$$

for all $x_{1}$ and $x_{2}$ in $I_{1}^{*}$. Defining

$$
\tilde{\mho}_{\mathfrak{x}}^{H}\left(x_{1}\right) \stackrel{\text { def }}{=} \int_{0}^{1} z \tilde{f}_{\mathfrak{x}}^{H}\left(x_{1} z\right) d z \quad \text { and } \quad \tilde{\mho}_{\mathfrak{x}}^{V}\left(x_{2}\right) \stackrel{\text { def }}{=} \int_{0}^{1} z \tilde{f}_{\mathfrak{x}}^{V}\left(x_{2} z\right) d z
$$

for all $x_{1}$ and $x_{2}$ in $I_{1}^{*}$, we have that

$$
\begin{aligned}
& \tilde{\Theta}_{\mathfrak{x}}\left(x_{1}, 0\right)=\int_{0}^{x_{1}} z \tilde{f}_{\mathfrak{x}}^{H}(z) d z=x_{1}^{2} \tilde{\mho}_{\mathfrak{x}}^{H}\left(x_{1}\right), \\
& \tilde{\Theta}_{\mathfrak{x}}\left(0, x_{2}\right)=-\int_{0}^{x_{2}} z \tilde{f}_{\mathfrak{x}}^{V}(z) d z=-x_{2}^{2} \tilde{\mho}_{\mathfrak{x}}^{V}\left(x_{2}\right),
\end{aligned}
$$

for all $x_{1}$ and $x_{2}$ in $I_{1}^{*}$.

Remark 2.14. Since $]$ is strictly positive, $\tilde{\mathrm{B}}_{\mathfrak{x}}$ is positive on $\square$, and $\tilde{g}_{\mathfrak{x}, 1,1}^{-1}$ and $\tilde{g}_{\mathfrak{x}, 2,2}^{-1}$ are strictly positive on $\square, \tilde{F}_{\mathfrak{x}}\left(0, x_{2}\right)$ and $\tilde{F}_{\mathfrak{x}}\left(x_{1}, 0\right)$ are strictly positive for $x_{1}$ and $x_{2}$ in $I_{1}^{*}$. Thus both $\tilde{f}_{\mathfrak{x}}^{H}$ and $\tilde{f}_{\mathfrak{x}}^{V}$ are strictly positive on $I_{1}^{*}$ and hence $\tilde{\Theta}_{\mathfrak{x}}\left(0, x_{2}\right) \leq 0$ and $\tilde{\Theta}_{\mathfrak{x}}\left(x_{1}, 0\right) \geq 0$ for all $x_{1}$ and $x_{2}$ in $I_{1}^{*}$. 
We want to extend $\bar{\Theta}$ away from $\boldsymbol{\Gamma}$. We will do so by means of a retract. We want this retract to interact nicely with $\bar{\Theta}$ near $\mathscr{X}$. To force this to happen, define

$$
\tilde{\Theta}_{\mathfrak{x}}\left(x_{1}, x_{2}\right) \stackrel{\text { def }}{=} x_{1}^{2} \tilde{\mho}_{\mathfrak{x}}^{H}\left(x_{1}\right)-x_{2}^{2} \tilde{\mho}_{\mathfrak{x}}^{V}\left(x_{2}\right)
$$

for all $\left(x_{1}, x_{2}\right) \in \check{\square}$.

LEMMA 2.15 (Retract) There is a retract $\wp: \mathrm{M} \rightarrow \boldsymbol{\Gamma}$ such that

(i) the set $\mathbf{X} \stackrel{\text { def }}{=}\{x \in M: \wp(x) \in \mathscr{X}\}$ is an immersed manifold of codimension 1 ,

(ii) $\wp$ is $C^{\infty}$ on $\mathrm{M} \backslash \mathbf{X}$,

(iii) for each $\mathfrak{x} \in \mathscr{X}, \bar{\Theta}_{\mathfrak{x}}^{\sim} \circ \phi_{\mathfrak{x}} \circ \wp$ is $C^{\infty}$ on $\wp^{-1}\left(\tilde{\phi}_{\mathfrak{x}}(\check{\square})\right)$, and

(iv) for each $\mathfrak{x} \in \mathscr{X}$, there is a neighborhood $\mathscr{V}_{\mathfrak{x}}$ of $\mathfrak{x}$ such that $\mathscr{V}_{\mathfrak{x}} \subset \tilde{\phi}_{\mathfrak{x}}(\check{\square})$ and $\overline{\wp\left(\mathscr{V}_{\mathfrak{x}}\right)} \subset \tilde{\phi}_{\mathfrak{x}}(\check{\square})$ and such that $\bar{\Theta}_{\mathfrak{x}}^{\sim} \circ \phi_{\mathfrak{x}} \circ \wp=\tilde{\Theta}_{\mathfrak{x}} \circ \phi_{\mathfrak{x}}$ on $\mathscr{V}_{\mathfrak{x}}$.

We shall give the proof of this result in Appendix A. Being a retract, $\wp$ is required to be continuous on $M$. For convenience, let $\varpi \in(0,1)$ be such that $\square \stackrel{\text { def }}{=}\left\{x \in \mathbb{R}^{2}\right.$ : $\left.\|x\|_{\infty}<\varpi\right\}$ is contained in $\bigcap_{\mathfrak{x} \in \mathscr{X}} \phi_{\mathfrak{x}}\left(\mathscr{V}_{\mathfrak{x}}\right)$. For each $\mathfrak{x} \in \mathscr{X}$, define $\mathbf{B}_{\mathfrak{x}} \stackrel{\text { def }}{=} \tilde{\phi}_{\mathfrak{x}}(\square)$. Define also $\mathcal{N} \stackrel{\text { def }}{=} M \backslash(\mathbf{X} \cup \boldsymbol{\Gamma})$, and for each $\mathfrak{x} \in \mathscr{X}$, define $\tilde{\mathcal{N}}_{\mathfrak{x}} \stackrel{\text { def }}{=} \phi_{\mathfrak{x}}\left(\mathbf{B}_{\mathfrak{x}} \cap \mathcal{N}\right)$.

We now define $\Theta(x) \stackrel{\text { def }}{=} \bar{\Theta} \circ \wp$ on $\mathrm{M}$ and for each $\ell \in \Lambda$, we define $\mathrm{C}_{\ell} \stackrel{\text { def }}{=} \overline{\mathbf{C}}_{\ell} \circ \wp$ and $\Theta_{\ell} \stackrel{\text { def }}{=} \Theta+\mathrm{C}_{\ell}$ on $\left(\mathrm{M}_{\ell} \cup \Gamma_{\ell}\right) \backslash \mathbf{X}$. For $\mathfrak{x} \in \mathscr{X}$, define

$$
\tilde{\Theta}_{\mathfrak{x}}^{\oplus}(x) \stackrel{\text { def }}{=} \sum_{\ell \in \Lambda} \Theta_{\ell}\left(\tilde{\phi}_{\mathfrak{x}}(x)\right) \chi_{\mathrm{M}_{\ell}}\left(\phi_{\mathfrak{x}}(x)\right)-\tilde{\Theta}_{\mathfrak{x}}(x), \quad x \in \tilde{\mathcal{N}}_{\mathfrak{x}},
$$

then $\tilde{\Theta}_{\mathfrak{x}}^{\oplus}$ is locally constant on $\tilde{\mathcal{N}}_{\mathfrak{x}}$; it is the correction function between $\tilde{\Theta}_{\mathfrak{x}}$ and the $\Theta_{\ell} \circ \tilde{\phi}_{\mathfrak{x}}$ 's.

LemMA 2.16 The function $\Theta$ is $C^{\infty}$ on $\mathrm{M} \backslash \mathbf{X}$. There is a constant $K>0$ such that for all $x \in \mathrm{M} \backslash \mathbf{X}$

$$
\begin{gathered}
|\Theta(x)| \leq K, \quad\|\nabla \Theta(x)\| \leq K, \quad|\mathscr{L} \Theta(x)| \leq K, \\
\left|(\bar{\nabla} \mathrm{H}, \nabla \Theta)(x)-I(x)\|\nabla \mathrm{H}(x)\|^{2}\right| \leq K|\mathrm{H}(x)|,
\end{gathered}
$$

and for all $\mathfrak{x} \in \mathscr{X}$ and $x \in \square$,

$$
\left|\tilde{\Theta}_{\mathfrak{x}}(x)\right| \leq K\|x\|_{\infty}^{2} \quad \text { and } \quad\left\|\nabla_{E} \tilde{\Theta}_{\mathfrak{x}}(x)\right\| \leq K\|x\|_{\infty} .
$$

The proof is in Appendix A.

For each $\ell$ and $\ell^{\prime}$ in $\Lambda$, define now $\gamma_{\ell, \ell^{\prime}} \stackrel{\text { def }}{=}\left(\Gamma_{\ell} \cap \Gamma_{\ell^{\prime}}\right) \backslash \mathscr{X}$. Fix $\ell$ and $\ell^{\prime}$ in $\Lambda$ such that $\gamma_{\ell, \ell^{\prime}} \neq \varnothing$. There is no assurance that $\Theta_{\ell}$ and $\Theta_{\ell^{\prime}}$ will agree on $\gamma_{\ell, \ell}$. Let's define a transition map in this case; namely, define $\iota_{\ell, \ell^{\prime}}(\theta) \stackrel{\text { def }}{=} \Theta_{\ell}\left(\mathbf{p}_{\ell^{\prime}}(\theta)\right.$ ) for all $\theta \in \Theta_{\ell^{\prime}}\left(\gamma_{\ell, \ell^{\prime}}\right)$. Recalling Remark 2.13, we have that $\iota_{\ell, \ell^{\prime}}$ is a diffeomorphism from $\Theta_{\ell^{\prime}}\left(\gamma_{\ell, \ell^{\prime}}\right)$ to $\Theta_{\ell}\left(\gamma_{\ell, \ell^{\prime}}\right)$. In fact, the map $\theta \mapsto \iota_{\ell, \ell^{\prime}}(\theta)-\theta$ is locally constant on $\Theta_{\ell^{\prime}}\left(\gamma_{\ell, \ell^{\prime}}\right)$ (on $\Theta_{\ell^{\prime}}\left(\gamma_{\ell, \ell^{\prime}}\right), \iota_{\ell, \ell^{\prime}}$ is $C^{\infty}$ and its derivative is identically 1$)$. 


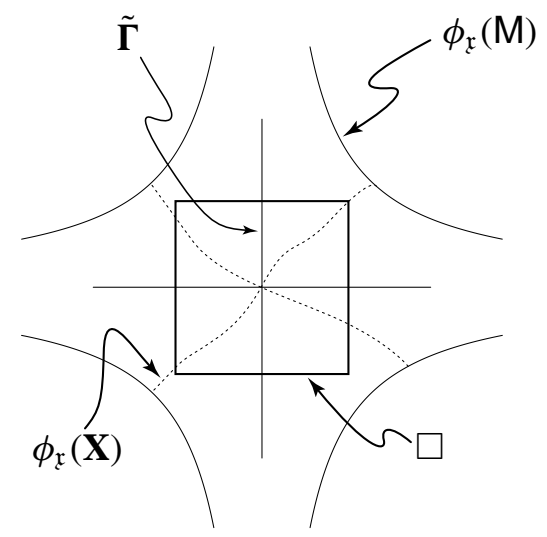

FIGURE 2.2. Local coordinates.

\section{Approximations}

We now construct several approximations to the $\Phi^{\varepsilon, \pm}$ 's of Theorem 2.6. If $\mathcal{O}$ is some open subset of $\mathrm{M} \backslash \mathbf{X}$ and $\psi \in C^{\infty}(\mathbb{R} \times \mathbb{R})$ and if we set $\psi^{\varepsilon}(x) \stackrel{\text { def }}{=}$ $\psi\left(\Theta(x), \frac{\mathrm{H}(x)}{\varepsilon}\right)$ for $x \in \mathcal{O}$ and $\varepsilon \in(0,1)$, then

$$
\begin{aligned}
\left(\mathscr{L}^{\varepsilon} \psi^{\varepsilon}\right)(x)= & \frac{1}{\varepsilon^{2}}\left\{\frac{\partial \psi}{\partial \theta}(\bar{\nabla} \mathrm{H}, \nabla \Theta)(x)+\frac{1}{2} \frac{\partial^{2} \psi}{\partial^{2} h} \beth\|\nabla \mathrm{H}\|^{2}(x)\right\} \\
& +\frac{1}{\varepsilon} \beth\left\{\frac{\partial \psi}{\partial h}(\mathscr{L} \mathrm{H})(x)+\frac{\partial^{2} \psi}{\partial \theta \partial h}(\nabla \mathrm{H}, \nabla \Theta)(x)\right\} \\
& +\beth\left\{\frac{\partial \psi}{\partial \theta}(\mathscr{L} \Theta)(x)+\frac{1}{2} \frac{\partial^{2} \psi}{\partial \theta^{2}}\|\nabla \Theta\|^{2}(x)\right\}
\end{aligned}
$$

for all $x \in \mathcal{O}$ and $\varepsilon \in(0,1)$, where the various derivatives of $\psi$ are all evaluated at $(\Theta(x), \mathrm{H}(x) / \varepsilon)$. The PDE (2.3) equates the coefficients of the dominant terms on $\boldsymbol{\Gamma}$, meaning that if $\mathscr{L}^{\varepsilon} \psi^{\varepsilon}$ is to be of order $o(1)$ on $\boldsymbol{\Gamma}$ as $\varepsilon \searrow 0$, then $\psi$ should satisfy the heat equation

$$
\frac{\partial \psi}{\partial \theta}+\frac{1}{2} \frac{\partial^{2} \psi}{\partial^{2} h}=0
$$

The significance of the following result is now partially clear: Define $\mathcal{R}_{\ell} \stackrel{\text { def }}{=}(0, \infty)$ if $\ell \in \Lambda_{P}$ and define $\mathcal{R}_{\ell} \stackrel{\text { def }}{=}(-\infty, 0)$ if $\ell \in \Lambda_{W}$.

PROPOSITION 3.1 There is a collection $\left\{\Psi_{\ell}: \ell \in \Lambda\right\}$ of functions such that the following hold:

(i) Periodicity COndition. For each $\ell \in \Lambda, \Psi_{\ell} \in C^{\infty}\left(\mathbb{R} \times \mathcal{R}_{\ell}\right)$ and

$$
\Psi_{\ell}(\theta, h)=\Psi_{\ell}\left(\theta+\mathscr{G}_{\ell}, h\right)
$$

for all $\theta \in \mathbb{R}$, and $h \in \mathcal{R}_{\ell}$. 
(ii) PDE CONDITION. For each $\ell \in \Lambda, \Psi_{\ell} \in C^{\infty}\left(\mathbb{R} \times \mathcal{R}_{\ell}\right)$ and

$$
\frac{\partial \Psi_{\ell}}{\partial \theta}(\theta, h)+\frac{1}{2} \frac{\partial^{2} \Psi_{\ell}}{\partial^{2} h}(\theta, h)=0
$$

for all $(\theta, h) \in \mathbb{R} \times \mathcal{R}_{\ell}$.

(iii) DECAY CONDITIONS. For each multiindex $\alpha$ such that $|\alpha| \geq 1$, there is a constant $K>0$ such that

$$
\left|D^{\alpha} \Psi_{\ell}(\theta, h)\right| \leq K \exp \left[-\sqrt{\frac{\pi}{\mathscr{G}_{\ell}}}|h|\right]
$$

for all $\ell \in \Lambda, \theta \in \mathbb{R}$, and $h \in \mathcal{R}_{\ell}$ such that $|h| \geq 1$.

(iv) MATCHING CONDITIONS AT THE BOUNDARIES. If $\ell$ and $\ell^{\prime}$ in $\Lambda$ are such that $\gamma_{\ell, \ell^{\prime}} \neq \varnothing$, then

$$
\lim _{\substack{h \rightarrow 0 \\ h \in \mathcal{R}_{\ell}}} \Psi_{\ell}(\cdot, h)=\lim _{\substack{h \rightarrow 0 \\ h \in \mathcal{R}_{\ell^{\prime}}}} \Psi_{\ell^{\prime}}\left(\iota_{\ell, \ell^{\prime}}(\cdot), h\right) \quad \text { in } L^{2}\left(\Theta_{\ell}\left(\gamma_{\ell, \ell^{\prime}}\right)\right)
$$

and

$$
\begin{aligned}
& \lim _{\substack{h \rightarrow 0 \\
h \in \mathcal{R}_{\ell}}} \int_{\theta \in \mathcal{I}_{\ell}}\left\{\frac{\partial \Psi_{\ell}}{\partial h}(\theta, h)+\mathrm{v}_{\ell}\right\} \varphi(\theta) d \theta= \\
& \lim _{\substack{h \rightarrow 0 \\
h \in \mathcal{R}_{\ell^{\prime}}}} \int_{\theta \in \mathcal{I}_{\ell}}\left\{\frac{\partial \Psi_{\ell^{\prime}}}{\partial h}\left(\iota_{\ell, \ell^{\prime}}(\theta), h\right)+\mathrm{v}_{\ell^{\prime}}\right\} \varphi(\theta) d \theta
\end{aligned}
$$

for all $\varphi \in C_{c}^{\infty}\left(\Theta_{\ell}\left(\gamma_{\ell, \ell^{\prime}}\right)\right)$.

(v) ENERGY ESTIMATE.

$$
\sum_{\ell \in \Lambda_{h \in \mathcal{R}_{\ell}}} \int_{\theta \in \mathcal{I}_{\ell}}\left(\frac{\partial \Psi_{\ell}}{\partial h}(\theta, h)\right)^{2} d \theta d h<\infty .
$$

The proof will be given in Appendix B. We can in fact do a bit better, thanks to well-known properties of the heat equation. It turns out that the $\Psi_{\ell}$ 's can have singularities near the points in $\mathbf{p}_{\ell}^{-1}(\mathscr{X}) \times\{0\}$. For each $\theta \in \mathbb{R}$, define $\mathrm{d}_{\ell}(\theta)=$ $\inf _{\theta^{\prime} \in \mathbf{p}_{\ell}^{-1}(\mathscr{X})}\left|\theta-\theta^{\prime}\right|$. Also, define $\stackrel{\mathscr{G}}{\stackrel{\text { def }}{=}} \max _{\ell \in \Lambda} \mathscr{G}_{\ell}$. For each nonnegative integer $k$, define

$$
\mathbf{s}_{k}(\theta, h) \stackrel{\text { def }}{=}\left\{\frac{1}{|\theta|^{k / 2}} \exp \left[-\frac{h^{2}}{4|\theta|}\right] \chi_{(0, \infty)}(\theta)+1\right\} \exp \left[-\sqrt{\frac{\pi}{\overline{\mathscr{G}}}}|h|\right]
$$

for all $\theta$ and $h \in \mathbb{R}$. The function $\mathbf{S}_{k}$ has exponential decay in $|h|$ for $|h|$ large and has a singularity of order $|\theta|^{-k / 2} e^{-h^{2} /(4|\theta|)}$ when $h$ and $\theta$ are near 0 . We also note that there is a constant $K>0$ such that

$$
\mathrm{s}_{k}(\theta, h) \leq \frac{K}{\left(|\theta|+h^{2}\right)^{k / 2}} \exp \left[-\sqrt{\frac{\pi}{\overline{\mathscr{G}}}}|h|\right]
$$

for all $k \in\{0, \ldots, 4\}$ and all $\theta \geq 0$ and $h \in \mathbb{R}$ such that $|\theta|+|h|>0$.

For $\ell \in \Lambda$, define $\hat{\digamma}_{\ell}(\theta, h) \stackrel{\text { def }}{=} \dot{f}_{\ell}(0) h$ for all $\theta \in \mathbb{R}$ and $h \in \mathcal{R}_{\ell}$. 
Proposition 3.2 Fix $\ell \in \Lambda$. Then there is a $K>0$ such that for every $n \in \mathbb{Z}$ and every multiindex $(j, k)$ with $j+k \leq 2$,

$$
\left|\frac{\partial^{j+k} \Psi_{\ell}}{\partial \theta^{j} \partial h^{k}}(\theta, h)\right| \leq K s_{2 j+k}\left(d_{\ell}(\theta), h\right)
$$

for all $\theta \in \mathbb{R}$ and $h \in \mathcal{R}_{\ell}$. For any $\ell$ and $\ell^{\prime}$ in $\Lambda$ such that $\gamma_{\ell, \ell^{\prime}} \neq \varnothing$, any multiindex $\alpha$, and all $\theta \in \Theta_{\ell}\left(\gamma_{\ell, \ell^{\prime}}\right)$,

$$
\lim _{\substack{h \rightarrow 0 \\ h \in \mathcal{R}_{\ell}}} D^{\alpha}\left(\Psi_{\ell}+\hat{\digamma}_{\ell}\right)(\theta, h)=\lim _{\substack{h \rightarrow 0 \\ h \in \mathcal{R}_{\ell^{\prime}}}} D^{\alpha}\left(\Psi_{\ell^{\prime}}+\hat{\digamma}_{\ell^{\prime}}\right)\left(\iota_{\ell, \ell^{\prime}}(\theta), h\right) .
$$

PROOF: The proof is essentially the same as that of proposition 8.7 of [9].

We can now make the basic part of the $\Phi^{\varepsilon, \pm}$ 's of Theorem 2.6. For $\varepsilon \in(0,1)$, define

$$
\Phi_{A}^{\varepsilon}(x) \stackrel{\text { def }}{=} \varepsilon \sum_{\ell \in \Lambda} \Psi_{\ell}\left(\Theta_{\ell}(x), \frac{\mathrm{H}(x)}{\varepsilon}\right) \chi_{\mathrm{M}_{\ell}}(x)
$$

for all $x \in \mathcal{N}$. We note that the prefactor of $\varepsilon$ is needed to get the appropriate Neumann data at $h=0$.

Lemma 3.3 For each $\varepsilon \in(0,1)$, the function $\Phi_{A}^{\varepsilon}+\digamma$ is $C^{1}$ at $(\boldsymbol{\Gamma} \cup \mathbf{X}) \backslash \mathscr{X}$.

Proof: Fix $\varepsilon \in(0,1)$. Next fix $x \in \boldsymbol{\Gamma} \backslash \mathscr{X}$. Then $x \in \gamma_{\ell, \ell^{\prime}}$ for some $\ell \in \Lambda_{P}$ and some $\ell \in \Lambda_{W}$. In a sufficiently small neighborhood $\mathcal{O}$ of $x$,

$$
\begin{aligned}
\Phi_{A}^{\varepsilon}(x)= & \varepsilon \Psi_{\ell}\left(\Theta_{\ell}(x), \frac{\mathrm{H}(x)}{\varepsilon}\right) \chi_{(0, \infty)}(\mathrm{H}(x)) \\
& +\varepsilon \Psi_{\ell^{\prime}}\left(\Theta_{\ell^{\prime}}(x), \frac{\mathrm{H}(x)}{\varepsilon}\right) \chi_{(-\infty, 0)}(\mathrm{H}(x)) \\
= & \varepsilon \Psi_{\ell}\left(\Theta_{\ell}(x), \frac{\mathrm{H}(x)}{\varepsilon}\right) \chi_{(0, \infty)}(\mathrm{H}(x)) \\
& +\varepsilon \Psi_{\ell^{\prime}}\left(\iota_{\ell^{\prime}, \ell}\left(\Theta_{\ell}(x)\right), \frac{\mathrm{H}(x)}{\varepsilon}\right) \chi_{(-\infty, 0)}(\mathrm{H}(x)),
\end{aligned}
$$

and we now use Proposition 3.2.

Next fix $x \in \mathbf{X} \backslash \mathscr{X}$. To show that $\Phi_{A}^{\varepsilon}$ is smooth at $x$, we will use some of the machinery of Appendix A; in particular, we use the notation of the proofs of Lemmas 2.11 and 2.15. Fix $x^{*} \in \mathrm{M}_{\ell}$ as at the start of the proof of Lemma 2.11. By Lemma A.2, there are $t^{*}$ and $\lambda^{*}$ in $\mathbb{R}$ such that $x=\wp_{\lambda^{*}}^{*}\left(\mathfrak{z}_{t^{*}}\left(x^{*}\right)\right)$. Since the map 
$(t, \lambda) \mapsto \wp_{\lambda^{*}}^{*}\left(\mathfrak{z}_{t^{*}}\left(x^{*}\right)\right)$ is full rank, it is a local diffeomorphism. Thus it is sufficient to verify that the map

$$
\begin{aligned}
f(t, \lambda) & \stackrel{\text { def }}{=} \Psi_{\ell}\left(\Theta_{\ell}\left(\wp_{\lambda^{*}}^{*}\left(\mathfrak{z}_{t^{*}}\left(x^{*}\right)\right)\right), \frac{\mathrm{H}\left(\wp_{\lambda^{*}}^{*}\left(\mathfrak{z}_{t^{*}}\left(x^{*}\right)\right)\right.}{\varepsilon}\right) \\
& \stackrel{\text { def }}{=} \Psi_{\ell}\left(\Theta_{\ell}\left(\wp\left(\mathfrak{z}_{t^{*}}\left(x^{*}\right)\right)\right), \frac{\mathrm{H}\left(x^{*}\right)(1-\lambda)}{\varepsilon}\right)
\end{aligned}
$$

is smooth at $\left(t^{*}, \lambda^{*}\right)$.

Define first

$$
R_{t} \stackrel{\text { def }}{=} \Theta_{\ell}\left(\wp\left(\mathfrak{z}_{t}\left(x^{*}\right)\right)\right)=\bar{\Theta}(q(t))+\overline{\mathrm{C}}_{\ell}(q(t))
$$

for all $t \in \mathbb{R}$. Then $R$ is $C^{\infty}$ on $\hat{A}$. Fix next $\mathfrak{x} \in \mathscr{X}$ and $t^{*} \in q^{-1}(\{\mathfrak{x}\}) \subset$ $\wp^{-1}\left(\tilde{\phi}_{\mathfrak{x}}(\square)\right)$. Then there is a neighborhood $\mathcal{O}$ of $t^{*}$ such that $\mathfrak{z}_{t}\left(x^{*}\right) \in \wp^{-1}\left(\phi_{\mathfrak{x}}(\square)\right)$ for $t \in \mathcal{O}$. Define next $\check{R}_{t} \stackrel{\text { def }}{=}\left(\bar{\Theta}_{\mathfrak{x}}^{\sim} \circ \phi_{\mathfrak{x}} \circ \wp\right)\left(\mathfrak{z}_{t}\left(x^{*}\right)\right)$ for $t \in \mathcal{O}$. Then $\check{R}$ is $C^{\infty}$ on $\mathcal{O}$ and $\check{R}-R$ is locally constant on $\mathcal{O} \backslash\left\{t^{*}\right\}$. Hence all derivatives of $R$ have well-defined limits at $t^{*}$.

Next define $\hat{t}^{*} \stackrel{\text { def }}{=} \tau\left(t^{*}\right)-\left\lfloor\tau\left(t^{*}\right) / \mathscr{G}_{\ell}\right\rfloor \mathscr{G}_{\ell} \in \mathcal{I}_{\ell}$. If $\hat{t}^{*} \in\left(0, \mathscr{G}_{\ell}\right)$, then

$$
\lim _{t \rightarrow t^{*}} R_{t}=\lim _{s \rightarrow \hat{t}^{*}}\left(\Theta_{\ell} \circ \mathbf{p}_{\ell}\right)(s)=\hat{t}^{*},
$$

in which case $R$ is continuous at $t^{*}$. If $\hat{t}^{*}=0$, then

$$
\lim _{t \searrow t^{*}} R_{t}=\lim _{s \searrow 0}\left(\Theta_{\ell} \circ \mathbf{p}_{\ell}\right)(s)=0 \quad \text { and } \quad \lim _{t \nearrow t^{*}} R_{t}=\lim _{s \nearrow \mathscr{G}_{\ell}}\left(\Theta_{\ell} \circ \mathbf{p}_{\ell}\right)(s)=\mathscr{G}_{\ell},
$$

in which case $R$ has a jump of size $\mathscr{G}_{\ell}$ at $t^{*}$. Combining things and using the $\mathscr{G}_{\ell^{-}}$ periodicity of $\Psi_{\ell}$ in its first argument, the desired smoothness becomes apparent.

This means that we can extend the domain of $\Phi_{A}^{\varepsilon}$ to $\mathrm{M} \backslash \mathscr{X}$. For $\varepsilon \in(0,1)$, define

$$
\begin{array}{cc}
\Phi_{A}^{\varepsilon}(x) \stackrel{\text { def }}{=} \lim _{\substack{x^{\prime} \rightarrow x \\
x^{\prime} \in \mathcal{N}}} \Phi_{A}^{\varepsilon}\left(x^{\prime}\right), & x \in \mathrm{M} \backslash \mathscr{X}, \\
\tilde{\Phi}_{A, \mathfrak{x}}^{\varepsilon}(x) \stackrel{\text { def }}{=} \Phi_{A}^{\varepsilon} \circ \tilde{\phi}_{\mathfrak{x}} & \text { on } \square \backslash\{\boldsymbol{0}\} \text { for } \mathfrak{x} \in \mathscr{X} .
\end{array}
$$

Fix now $\mathfrak{x} \in \mathscr{X}$. We need to modify things at $\mathfrak{x}$ itself due to the singularities of the $\Psi_{\ell}$ 's near $\mathbf{p}_{\ell}^{-1}(\mathscr{X}) \times\{0\}$ (as characterized in Proposition 3.2). We start by returning to $(\theta, h)$-coordinates. Note that there are four (not necessarily distinct) indices $\ell_{1}(\mathfrak{x}), \ell_{2}(\mathfrak{x}), \ell_{3}(\mathfrak{x})$, and $\ell_{4}(\mathfrak{x})$ in $\Lambda$ such that $\mathfrak{x} \in \bigcap_{i=1}^{4} \overline{\mathrm{M}_{\ell_{1}(\mathfrak{x})}}$ and such that $\phi_{\mathfrak{x}}\left(\mathrm{M}_{\ell_{1}(\mathfrak{x})} \cap \mathbf{B}_{\mathfrak{x}}\right) \subset \mathbb{R}_{+} \times \mathbb{R}_{+}, \phi_{\mathfrak{x}}\left(\mathrm{M}_{\ell_{2}(\mathfrak{x})} \cap \mathbf{B}_{\mathfrak{x}}\right) \subset \mathbb{R}_{-} \times \mathbb{R}_{+}, \phi_{\mathfrak{x}}\left(\mathrm{M}_{\ell_{3}(\mathfrak{x})} \cap \mathbf{B}_{\mathfrak{x}}\right) \subset \mathbb{R}_{-} \times \mathbb{R}_{-}$, and $\phi_{\mathfrak{x}}\left(\mathrm{M}_{\ell_{4}(\mathfrak{x})} \cap \mathbf{B}_{\mathfrak{x}}\right) \subset \mathbb{R}_{+} \times \mathbb{R}_{-}$. The vector field $\nabla_{E} \tilde{\mathrm{H}}$ points inward along the vertical axis $\{0\} \times \mathbb{R}$ and points outward along the horizontal axis $\mathbb{R} \times\{0\}$; the stable manifold of $\mathfrak{z}$ at $\mathfrak{x}$ contains the $\tilde{\phi}_{\mathfrak{x}}$ image of $\{0\} \times \mathbb{R}$, and the unstable manifold of $\mathfrak{z}$ at $\mathfrak{x}$ contains the $\tilde{\phi}_{\mathfrak{x}}$ image of $\mathbb{R} \times\{0\}$. Note that

$$
\left\{x \in \square: \tilde{\Theta}_{\mathfrak{x}}(x)=0\right\}=\left\{x \in \square:\left|x_{1}\right| \tilde{\mho}_{\mathfrak{x}}^{H}\left(x_{1}\right)=\left|x_{2}\right| \tilde{\mho}_{\mathfrak{x}}^{V}\left(x_{2}\right)\right\} ;
$$


then define

$$
\begin{aligned}
& \mathcal{C}_{\mathfrak{x}, U} \stackrel{\text { def }}{=}\left\{\left(x_{1}, x_{2}\right) \in \square: x_{2} \tilde{\mho}_{\mathfrak{x}}^{V}\left(x_{2}\right)>\left|x_{1}\right| \tilde{\mho}_{\mathfrak{x}}^{H}\left(x_{1}\right)\right\}, \\
& \mathcal{C}_{\mathfrak{x}, D} \stackrel{\text { def }}{=}\left\{\left(x_{1}, x_{2}\right) \in \square: x_{2} \tilde{\mho}_{\mathfrak{x}}^{V}\left(x_{2}\right)<-\left|x_{1}\right| \tilde{\mho}_{\mathfrak{x}}^{H}\left(x_{1}\right)\right\}, \\
& \mathcal{C}_{\mathfrak{x}, R} \stackrel{\text { def }}{=}\left\{\left(x_{1}, x_{2}\right) \in \square: x_{1} \tilde{\mho}_{\mathfrak{x}}^{Y}\left(x_{1}\right)>\left|x_{2}\right| \tilde{\mho}_{\mathfrak{x}}^{H}\left(x_{2}\right)\right\}, \\
& \mathcal{C}_{\mathfrak{x}, L} \stackrel{\text { def }}{=}\left\{\left(x_{1}, x_{2}\right) \in \square: x_{1} \tilde{\mho}_{\mathfrak{x}}^{V}\left(x_{1}\right)<-\left|x_{2}\right| \tilde{\mho}_{\mathfrak{x}}^{H}\left(x_{2}\right)\right\} .
\end{aligned}
$$

Set

$$
\begin{aligned}
& \mho_{+} \stackrel{\text { def }}{=} \sup \left\{\tilde{\mho}_{\mathfrak{x}}^{s}(x):|x|<\varpi, \mathfrak{x} \in \mathscr{X}, s \in\{H, V\}\right\}, \\
& \mho_{-} \stackrel{\text { def }}{=} \inf \left\{\tilde{\mho}_{\mathfrak{x}}^{s}(x):|x|<\varpi, \mathfrak{x} \in \mathscr{X}, s \in\{H, V\}\right\} ;
\end{aligned}
$$

then $0<\mho_{-} \leq \mho_{+}<\infty$ and

$$
\begin{aligned}
& \mathcal{C}_{\mathfrak{x}, U} \cup \mathcal{C}_{\mathfrak{x}, D} \subset\left\{\left(x_{1}, x_{2}\right) \in \mathbb{R}^{2}:\left|x_{1}\right| \leq \frac{\mho_{+}}{\mho_{-}}\left|x_{2}\right|\right\}, \\
& \mathcal{C}_{\mathfrak{x}, L} \cup \mathcal{C}_{\mathfrak{x}, R} \subset\left\{\left(x_{1}, x_{2}\right) \in \mathbb{R}^{2}:\left|x_{2}\right| \leq \frac{\mho_{+}}{\mho_{-}}\left|x_{1}\right|\right\} .
\end{aligned}
$$

For each $i \in\{1,2,3,4\}$, define

$$
\mathfrak{c}_{i}(\mathfrak{x}) \stackrel{\text { def }}{=} \varlimsup_{\substack{x^{\prime} \in \Gamma_{\ell_{i}(\mathfrak{x})} \\ x^{\prime} \rightarrow \mathfrak{x}}} \Theta_{\ell}\left(x^{\prime}\right)
$$

(we could alternately have used the $\underline{\mathrm{lim}}$; by Lemma 2.12 , either the $\underline{\lim }$ and $\overline{\mathrm{lim}}$ agree or differ by exactly $\mathscr{G}_{\ell}$ ). Defining now $\underline{\mathscr{G}} \stackrel{\text { def }}{=} \min _{\ell \in \Lambda} \mathscr{G}_{\ell}$, we define the four functions $u_{\mathfrak{x}, L}, u_{\mathfrak{x}, R}, u_{\mathfrak{x}, U}$, and $u_{\mathfrak{x}, D}$ by

$$
\begin{aligned}
& u_{\mathfrak{x}, U}(t, h) \stackrel{\text { def }}{=} \begin{cases}\Psi_{\ell_{1}(\mathfrak{x})}\left(\mathfrak{c}_{1}(\mathfrak{x})-t, h\right)+\mathrm{v}_{\ell_{1}(\mathfrak{x})} h & \text { if }(t, h) \in(0, \underline{\mathscr{G}}) \times(0, \infty) \\
\Psi_{\ell_{2}(\mathfrak{x})}\left(\mathfrak{c}_{2}(\mathfrak{x})-t, h\right)+\mathrm{v}_{\ell_{2}(\mathfrak{x})} h & \text { if }(t, h) \in(0, \underline{G}) \times(-\infty, 0),\end{cases} \\
& u_{\mathfrak{x}, D}(t, h) \stackrel{\text { def }}{=} \begin{cases}\Psi_{\ell_{3}(\mathfrak{x})}\left(\mathfrak{c}_{3}(\mathfrak{x})-t, h\right)+\mathrm{v}_{\ell_{3}(\mathfrak{x})} h & \text { if }(t, h) \in(0, \underline{G}) \times(0, \infty) \\
\Psi_{\ell_{4}(\mathfrak{x})}\left(\mathfrak{c}_{4}(\mathfrak{x})-t, h\right)+\mathrm{v}_{\ell_{4}(\mathfrak{x})} h & \text { if }(t, h) \in(0, \underline{G}) \times(-\infty, 0),\end{cases} \\
& u_{\mathfrak{x}, L}(t, h) \stackrel{\text { def }}{=} \begin{cases}\Psi_{\ell_{2}(\mathfrak{x})}\left(\mathfrak{c}_{2}(\mathfrak{x})-t, h\right)+\mathrm{v}_{\ell_{2}(\mathfrak{x})} h & \text { if }(t, h) \in(-\underline{G}, 0) \times(-\infty, 0) \\
\Psi_{\ell_{3}(\mathfrak{x})}\left(\mathfrak{c}_{3}(\mathfrak{x})-t, h\right)+\mathrm{v}_{\ell_{3}(\mathfrak{x})} h & \text { if }(t, h) \in(-\underline{\mathscr{G}}, 0) \times(0, \infty),\end{cases} \\
& u_{\mathfrak{x}, R}(t, h) \stackrel{\text { def }}{=} \begin{cases}\Psi_{\ell_{1}(\mathfrak{x})}\left(\mathfrak{c}_{1}(\mathfrak{x})-t, h\right)+\mathrm{v}_{\ell_{1}(\mathfrak{x})} h & \text { if }(t, h) \in(-\underline{\mathscr{G}}, 0) \times(0, \infty) \\
\Psi_{\ell_{4}(\mathfrak{x})}\left(\mathfrak{c}_{4}(\mathfrak{x})-t, h\right)+\mathrm{v}_{\ell_{4}(\mathfrak{x})} h & \text { if }(t, h) \in(-\underline{\mathscr{G}}, 0) \times(-\infty, 0)\end{cases}
\end{aligned}
$$

(note that $\tilde{\Theta}_{\mathfrak{x}}$ is positive on the horizontal axis in $\square$ and negative on the vertical axis of $\square$ ); see Remark 2.14-this is the reason for choosing $(-\underline{G}, 0)$ for $u_{\mathfrak{x}, L}$ and 
$u_{\mathfrak{x}, R}$ and $(0, \underline{\mathscr{G}})$ for $u_{\mathfrak{x}, U}$ and $\left.u_{\mathfrak{x}, D}\right)$. Defining $\tilde{\digamma}_{\mathfrak{x}} \stackrel{\text { def }}{=} \digamma \circ \tilde{\phi}_{\mathfrak{x}}$ on $\square$, we have that

$$
\tilde{\Phi}_{A, \mathfrak{x}}^{\varepsilon}(x)+\tilde{\digamma}_{\mathfrak{x}}(x)=\varepsilon \sum_{s \in\{L, R, U, D\}} u_{\mathfrak{x}, s}\left(-\tilde{\Theta}_{\mathfrak{x}}(x), \frac{\tilde{\mathrm{H}}(x)}{\varepsilon}\right) \chi_{\mathcal{C}_{\mathfrak{x}, s}}(x)
$$

for $\varepsilon \in(0,1)$ and $x \in \tilde{\mathcal{N}}_{\mathfrak{x}}$.

Note that we can define the $u_{\mathfrak{x}, s}$ 's at $h=0$ by continuity (this is possible due to Lemma 3.3); i.e.,

$$
u_{\mathfrak{x}, s}(t, 0)=\lim _{\substack{h \rightarrow 0 \\ h \neq 0}} u_{\mathfrak{x}, s}(t, h)
$$

for $t \in(0, \underline{G})$ if $s \in\{U, D\}$ and for $t \in(-\underline{G}, 0)$ if $s \in\{L, R\}$. By (3.2), we have that for all $s \in\{L, R, U, D\}$,

$$
\frac{\partial u_{\mathfrak{r}, s}}{\partial t}(t, h)=\frac{1}{2} \frac{\partial^{2} u_{\mathfrak{r}, s}}{\partial h^{2}}(t, h)
$$

on $(0, \underline{\mathscr{G}}) \times \mathbb{R}$ if $s \in\{U, D\}$ and on on $(-\underline{G}, 0) \times \mathbb{R}$ if $s \in\{L, R\}$. From Proposition 3.2 (or alternately by using the fact that the heat equation smooths in forward time), the function

$$
u_{\mathfrak{x}, s}^{\circ}(h) \stackrel{\text { def }}{=} \lim _{t \nearrow 0} u_{\mathfrak{x}, s}(t, h), \quad h \in \mathbb{R},
$$

exists and is in $C^{\infty}$ for $s \in\{L, R\}$. Defining

$$
\begin{aligned}
& u_{\mathfrak{x}, U}^{\circ}(h)= \begin{cases}u_{\mathfrak{x}, R}^{\circ}(h) & \text { if } h>0 \\
u_{\mathfrak{x}, L}^{\circ}(h) & \text { if } h<0,\end{cases} \\
& u_{\mathfrak{x}, D}^{\circ}(h)= \begin{cases}u_{\mathfrak{x}, L}^{\circ}(h) & \text { if } h>0 \\
u_{\mathfrak{x}, R}^{\circ}(h) & \text { if } h<0,\end{cases}
\end{aligned}
$$

we also have that

$$
u_{\mathfrak{x}, s}^{\circ}(h) \stackrel{\text { def }}{=} \lim _{t \searrow 0} u_{\mathfrak{x}, s}(t, h), \quad h \in \mathbb{R} \backslash\{0\},
$$

for $s \in\{U, D\}$. Thus $\left.u_{\mathfrak{x}, s}^{\circ}\right|_{\mathbb{R}_{+}} \in C^{\infty}\left(\mathbb{R}_{+}\right)$and $\left.u_{\mathfrak{y}, s}^{\circ}\right|_{\mathbb{R}_{-}} \in C^{\infty}\left(\mathbb{R}_{-}\right)$for $s \in\{U, D\}$.

Remark 3.4. There is no assurance that

$$
\frac{d^{n} u_{\mathfrak{x}, U}^{\circ}}{d h^{n}}(0+)=\frac{d^{n} u_{\mathfrak{x}, U}^{\circ}}{d h^{n}}(0-) \quad \text { or } \quad \frac{d^{n} u_{\mathfrak{x}, D}^{\circ}}{d h^{n}}(0+)=\frac{d^{n} u_{\mathfrak{x}, D}^{\circ}}{d h^{n}}(0-) .
$$

Thus we can have singularities in $u_{\mathfrak{x}, U}$ and $u_{\mathfrak{x}, D}$ at $(0,0)$, but $u_{\mathfrak{x}, R}$ and $u_{\mathfrak{x}, L}$ are smooth. Equivalently, this means that $\tilde{\Phi}_{\mathfrak{x}, A}^{\varepsilon}$ may have (complicated) singularities at the origin in $\mathcal{C}_{\mathfrak{x}, U} \cup \mathcal{C}_{\mathfrak{x}, D}$.

Thus we should look a bit more closely at $\tilde{\Phi}_{A, \mathfrak{x}}^{\varepsilon}$ in $\mathcal{C}_{\mathfrak{x}, U}$ and $\mathcal{C}_{\mathfrak{x}, D}$. From the standpoint of singular perturbations, this is natural; we need to make something of 
"corner expansion" near 0. This corner expansion should be valid when $\tilde{\Theta}_{\mathfrak{x}}$ and $|\tilde{\mathrm{H}} / \varepsilon|$ are small. If $\left(x_{1}, x_{2}\right) \in \mathcal{C}_{\mathfrak{x}, U} \cup \mathcal{C}_{\mathfrak{x}, D}$ and $\varepsilon \in(0,1)$, then

$$
\left|x_{1}\right|=\sqrt{\left|x_{1}\right|\left|x_{1}\right|} \leq \sqrt{\frac{\mho_{+}}{\mho_{-}}\left|\tilde{\mathrm{H}}\left(x_{1}, x_{2}\right)\right|} \leq \sqrt{\varepsilon \frac{\mho_{+}}{\mho_{-}}\left|\frac{\tilde{\mathrm{H}}\left(x_{1}, x_{2}\right)}{\varepsilon}\right|},
$$

so if $\left(x_{1}, x_{2}\right) \in \mathcal{C}_{\mathfrak{x}, U} \cup \mathcal{C}_{\mathfrak{x}, D}$ and $\left|\tilde{\mathrm{H}}\left(x_{1}, x_{2}\right) / \varepsilon\right|$ is small, then $\left|x_{1}\right|$ is small (i.e., we are near the vertical part of $\tilde{\boldsymbol{\Gamma}})$, and we should be able to replace the coordinate $\tilde{\Theta}_{\mathfrak{x}}\left(x_{1}, x_{2}\right)$ by $\tilde{\Theta}_{\mathfrak{x}}\left(0, x_{2}\right)$. To carry this out, for $\varepsilon \in(0,1)$ define for $\left(x_{1}, x_{2}\right) \in \square$ such that $x_{2} \neq 0$

$$
\tilde{\Phi}_{B, \mathfrak{x}}^{\varepsilon}\left(x_{1}, x_{2}\right) \stackrel{\text { def }}{=} \begin{cases}\varepsilon u_{\mathfrak{x}, U}\left(-\tilde{\Theta}_{\mathfrak{x}}\left(0, x_{2}\right), \frac{\tilde{\mathrm{H}}\left(x_{1}, x_{2}\right)}{\varepsilon}\right) & \text { if } x_{2}>0 \\ \varepsilon u_{\mathfrak{x}, D}\left(-\tilde{\Theta}_{\mathfrak{x}}\left(0, x_{2}\right), \frac{\tilde{\mathrm{H}}\left(x_{1}, x_{2}\right)}{\varepsilon}\right) & \text { if } x_{2}<0\end{cases}
$$

and define for all $x \in \mathbf{B}_{\mathfrak{x}}$ such that $\phi_{\mathfrak{x}}(x) \notin \mathbb{R} \times\{0\}$

$$
\Phi_{B, \mathfrak{x}}^{\varepsilon}(x) \stackrel{\text { def }}{=} \tilde{\Phi}_{B, \mathfrak{x}}^{\varepsilon}\left(\phi_{\mathfrak{x}}(x)\right) .
$$

To study $\tilde{\Phi}_{B, \mathfrak{x}}^{\varepsilon}$, define

$$
\tilde{\vartheta}_{\mathfrak{x}, 1}\left(x_{1}, x_{2}\right) \stackrel{\text { def }}{=} \frac{x_{1}}{\sqrt{\tilde{\mho}_{\mathfrak{x}}^{V}\left(x_{2}\right)}} \text { and } \quad \tilde{\vartheta}_{\mathfrak{x}, 2}\left(x_{1}, x_{2}\right) \stackrel{\text { def }}{=} x_{2} \sqrt{\tilde{\mho}_{\mathfrak{x}}^{V}\left(x_{2}\right)}
$$

for all $\left(x_{1}, x_{2}\right) \in \square$. For $z \in \mathbb{R}$, define $\mathfrak{s}(z) \stackrel{\text { def }}{=} z /|z|$ if $z \neq 0$, and set $\mathfrak{s}(0) \stackrel{\text { def }}{=} 0$. Then

$$
\sqrt{-\tilde{\Theta}_{\mathfrak{x}}\left(0, x_{2}\right)}=\mathfrak{s}\left(x_{2}\right) \tilde{\vartheta}_{\mathfrak{x}, 2}\left(x_{1}, x_{2}\right) \text { and } \frac{\tilde{\mathrm{H}}\left(x_{1}, x_{2}\right)}{\sqrt{-\tilde{\Theta}_{\mathfrak{x}}\left(0, x_{2}\right)}}=\mathfrak{s}\left(x_{2}\right) \tilde{\vartheta}_{\mathfrak{x}, 1}\left(x_{1}, x_{2}\right)
$$

for all $\left(x_{1}, x_{2}\right) \in \square$ such that $x_{2} \neq 0$. Define also the heat kernel

$$
\mathfrak{g}(z) \stackrel{\text { def }}{=} \frac{1}{\sqrt{2 \pi}} e^{-\frac{z^{2}}{2}}, \quad z \in \mathbb{R} .
$$

Lemma 3.5 For $\varepsilon \in(0,1), \tilde{\Phi}_{B, \mathfrak{x}}^{\varepsilon}+\tilde{\digamma}_{\mathfrak{x}}$ is smooth at $\left\{\left(x_{1}, x_{2}\right) \in \square: x_{2}=0\right\}$ and furthermore

$$
\begin{aligned}
\tilde{\Phi}_{B, \mathfrak{x}}^{\varepsilon}(x)+\tilde{\digamma}_{\mathfrak{x}}(x)= & \varepsilon \int_{z=0}^{\infty} \mathfrak{g}\left(\frac{\tilde{\vartheta}_{\mathfrak{x}, 1}(x)}{\varepsilon}-z\right) u_{\mathfrak{x}, R}^{\circ}\left(\tilde{\vartheta}_{\mathfrak{x}, 2}(x) z\right) d z \\
& +\varepsilon \int_{z=-\infty}^{0} \mathfrak{g}\left(\frac{\tilde{\vartheta}_{\mathfrak{x}, 1}(x)}{\varepsilon}-z\right) u_{\mathfrak{x}, L}^{\circ}\left(\tilde{\vartheta}_{\mathfrak{x}, 2}(x) z\right) d z
\end{aligned}
$$

for all $x=\left(x_{1}, x_{2}\right) \in \square$ such that $x_{2} \neq 0$. 
PROOF: We recall (3.8) and use (3.9). We also use $\mathfrak{g}$ to explicitly solve the heat equation (3.7). Fix $\varepsilon$ and $x$ as required. If $x_{2}>0$ (hence $\sqrt{-\tilde{\Theta}_{\mathfrak{x}}\left(0, x_{2}\right)}=\tilde{\vartheta}_{\mathfrak{x}, 2}(x)$ ),

$$
\begin{aligned}
\tilde{\Phi}_{C, \mathfrak{x}}^{\varepsilon}(x)+\tilde{\digamma}_{\mathfrak{x}}(x) & =\varepsilon \int_{z \in \mathbb{R}} \frac{1}{\sqrt{-\tilde{\Theta}_{\mathfrak{x}}\left(0, x_{2}\right)}} \mathfrak{g}\left(\frac{\tilde{\mathrm{H}}(x) / \varepsilon-z}{\sqrt{-\tilde{\Theta}_{\mathfrak{x}}\left(0, x_{2}\right)}}\right) u_{\mathfrak{x}, U}^{\circ}(z) d z \\
& =\varepsilon \int_{z \in \mathbb{R}} \mathfrak{g}\left(\frac{\tilde{\mathrm{H}}(x)}{\varepsilon \sqrt{-\tilde{\Theta}_{\mathfrak{x}}\left(0, x_{2}\right)}}-z\right) u_{\mathfrak{x}, U}^{\circ}\left(\tilde{\vartheta}_{\mathfrak{x}, 2}(x) z\right) d z
\end{aligned}
$$

and this agrees with (3.10). If $x_{2}<0$ (hence $\left.\sqrt{-\tilde{\Theta}_{\mathfrak{x}}\left(0, x_{2}\right)}=-\tilde{\vartheta}_{\mathfrak{x}, 2}(x)\right)$,

$$
\begin{aligned}
\tilde{\Phi}_{B, \mathfrak{x}}^{\varepsilon}(x)+\tilde{\digamma}_{\mathfrak{x}}(x)= & \varepsilon \int_{z \in \mathbb{R}} \frac{1}{\sqrt{-\tilde{\Theta}_{\mathfrak{x}}\left(0, x_{2}\right)}} \mathfrak{g}\left(\frac{\tilde{\mathrm{H}}(x) / \varepsilon-z}{\sqrt{-\tilde{\Theta}_{\mathfrak{x}}\left(0, x_{2}\right)}}\right) u_{\mathfrak{x}, D}^{\circ}(z) d z \\
= & \varepsilon \int_{z \in \mathbb{R}} \mathfrak{g}\left(\frac{\tilde{\mathrm{H}}(x)}{\varepsilon \sqrt{-\tilde{\Theta}_{\mathfrak{x}}\left(0, x_{2}\right)}}-z\right) u_{\mathfrak{x}, D}^{\circ}\left(-\tilde{\vartheta}_{\mathfrak{x}, 2}(x) z\right) d z \\
= & \varepsilon \int_{z=0}^{\infty} \mathfrak{g}\left(-\frac{\tilde{\vartheta}_{\mathfrak{x}, 1}(x)}{\varepsilon}-z\right) u_{\mathfrak{x}, L}^{\circ}\left(-\tilde{\vartheta}_{\mathfrak{x}, 2}(x) z\right) d z \\
& +\varepsilon \int_{z=-\infty}^{0} \mathfrak{g}\left(-\frac{\tilde{\vartheta}_{\mathfrak{x}, 1}(x)}{\varepsilon}-z\right) u_{\mathfrak{x}, R}^{\circ}\left(-\tilde{\vartheta}_{\mathfrak{x}, 2}(x) z\right) d z \\
= & \varepsilon \int_{z=-\infty}^{0} \mathfrak{g}\left(-\frac{\tilde{\vartheta}_{\mathfrak{x}, 1}(x)}{\varepsilon}+z\right) u_{\mathfrak{x}, L}^{\circ}\left(\tilde{\vartheta}_{\mathfrak{x}, 2}(x) z\right) d z \\
& +\varepsilon \int_{z=0}^{\infty} \mathfrak{g}\left(-\frac{\tilde{\vartheta}_{\mathfrak{x}, 1}(x)}{\varepsilon}+z\right) u_{\mathfrak{x}, R}^{\circ}\left(\tilde{\vartheta}_{\mathfrak{x}, 2}(x) z\right) d z,
\end{aligned}
$$

and we now use the fact that $\mathfrak{g}$ is even; again, this agrees with (3.10). We now have the stated representation of $\tilde{\Phi}_{B, \mathfrak{x}}^{\varepsilon}$. The stated regularity follows easily from this representation; note that $\tilde{\vartheta}_{1, \mathfrak{x}}$ and $\tilde{\vartheta}_{2, \mathfrak{x}}$ are smooth.

For $\varepsilon \in(0,1)$, we define

$$
\begin{array}{cc}
\tilde{\Phi}_{B, \mathfrak{x}}^{\varepsilon}(x) \stackrel{\text { def }}{=} \lim _{\substack{\left(x_{1}, x_{2}\right) \rightarrow x \\
x_{2} \neq 0 \\
\left(x_{1}, x_{2}\right) \in \square}} \tilde{\Phi}_{B, \mathfrak{x}}^{\varepsilon}\left(x_{1}, x_{2}\right), \quad x \in \square, \\
\Phi_{B, \mathfrak{x}}^{\varepsilon} \stackrel{\text { def }}{=} \tilde{\Phi}_{B, \mathfrak{x}}^{\varepsilon} \circ \phi_{\mathfrak{x}} & \text { on } \mathbf{B}_{\mathfrak{x}} .
\end{array}
$$

We will later, in Lemma 4.3, use (3.10) to show that $\mathscr{L}^{\varepsilon} \Phi_{\mathfrak{x}, B}^{\varepsilon}$ is small. 
Near $\mathfrak{x}$, we want to use $\Phi_{B, \mathfrak{x}}^{\varepsilon}$ instead of $\Phi_{A}^{\varepsilon}$. In view of the heat singularities that occur near $\mathfrak{x}$, define

$$
\begin{aligned}
& \tilde{\mathfrak{D}}_{\mathfrak{x}}^{\varepsilon}(x) \stackrel{\text { def }}{=}\left\{\left|\tilde{\Theta}_{\mathfrak{x}}(x)\right|^{2}+\left|\frac{\tilde{\mathrm{H}}(x)}{\varepsilon}\right|^{4}\right\}^{\frac{1}{2}}, \quad x \in \square, \\
& \mathfrak{D}_{\mathfrak{x}}^{\varepsilon}(x) \stackrel{\text { def }}{=} \tilde{\mathfrak{D}}_{\mathfrak{x}}^{\varepsilon}\left(\tilde{\phi}_{\mathfrak{x}}(x)\right), \quad x \in \mathbf{B}_{\mathfrak{x}} .
\end{aligned}
$$

We can compare $\tilde{\mathfrak{D}}_{\mathfrak{x}}^{\varepsilon}$ to the Euclidean norm via the following:

LEMMA 3.6 There is a constant $K_{3.6}>0$ such that for all $\varepsilon \in(0,1), \mathfrak{x} \in \mathscr{X}$, and $x \in \square$,

$$
\begin{gathered}
\|x\|_{\infty} \leq K_{3.6}\left\{\left(\tilde{\mathfrak{D}}_{\mathfrak{x}}^{\varepsilon}(x)\right)^{\frac{1}{2}}+\left(\tilde{\mathfrak{D}}_{\mathfrak{x}}^{\varepsilon}(x)\right)^{\frac{1}{4}}\right\} \\
\|x\|_{\infty} \leq K_{3.6}\left\{\left(\tilde{\mathfrak{D}}_{\mathfrak{x}}^{\varepsilon}(x)\right)^{\frac{1}{2}}+\varepsilon\right\}, \quad \text { and } \quad|\tilde{\mathrm{H}}(x)| \leq \varepsilon\left(\tilde{\mathfrak{D}}_{\mathfrak{x}}^{\varepsilon}(x)\right)^{\frac{1}{2}}
\end{gathered}
$$

ProOF: Fix $\varepsilon, \mathfrak{x}$, and $x$ as required. To see the last bound, we simply write that

$$
|\tilde{\mathrm{H}}(x)|=\varepsilon\left|\frac{\tilde{\mathrm{H}}(x)}{\varepsilon}\right| \leq \varepsilon \sqrt{\tilde{\mathfrak{D}}_{\mathfrak{x}}^{\varepsilon}(x)} .
$$

We next observe that

$$
\begin{aligned}
\|x\|_{\infty}^{4} \leq x_{1}^{4}+x_{2}^{4} & \leq \frac{1}{\mho_{-}^{2}}\left\{\left(x_{1}^{2} \tilde{\mho}_{\mathfrak{x}}^{H}\left(x_{1}\right)\right)^{2}+\left(x_{2}^{2} \tilde{\mho}_{\mathfrak{x}}^{V}\left(x_{2}\right)\right)^{2}\right\} \\
& \leq \frac{1}{\mho_{-}^{2}}\left\{\left(x_{1}^{2} \tilde{\mho}_{\mathfrak{x}}^{H}\left(x_{1}\right)-x_{2}^{2} \tilde{\mho}_{\mathfrak{x}}^{V}\left(x_{2}\right)\right)^{2}+2 x_{1}^{2} x_{2}^{2} \tilde{\mho}_{\mathfrak{x}}^{H}\left(x_{1}\right) \tilde{\mho}_{\mathfrak{x}}^{V}\left(x_{2}\right)\right\} \\
& \leq \frac{1}{\mho_{-}^{2}}\left\{\left(\tilde{\Theta}_{\mathfrak{x}}\left(x_{1}, x_{2}\right)\right)^{2}+2 \mho_{+}^{2} \tilde{H}^{2}\left(x_{1}, x_{2}\right)\right\} \\
& \leq \frac{1}{\mho_{-}^{2}}\left\{\left(\tilde{\mathfrak{D}}_{\mathfrak{x}}^{\varepsilon}(x)\right)^{2}+2 \mho \mho_{+}^{2} \varepsilon^{2} \tilde{\mathfrak{D}}_{\mathfrak{x}}^{\varepsilon}(x)\right\}
\end{aligned}
$$

and hence

$$
\begin{aligned}
\|x\|_{\infty} & \leq \frac{1}{\sqrt{\mho_{-}}} \max \left\{1,2^{\frac{1}{4}} \sqrt{\mho_{+}}\right\}\left\{\left(\tilde{\mathfrak{D}}_{\mathfrak{x}}^{\varepsilon}(x)\right)^{2}+\varepsilon^{2} \tilde{\mathfrak{D}}_{\mathfrak{x}}^{\varepsilon}(x)\right\}^{\frac{1}{4}} \\
& \leq \frac{2^{1 / 4}}{\sqrt{\mho_{-}}} \max \left\{1,2^{\frac{1}{4}} \sqrt{\mho_{+}}\right\}\left\{\left(\tilde{\mathfrak{D}}_{\mathfrak{x}}^{\varepsilon}(x)\right)^{\frac{1}{2}}+\varepsilon^{\frac{1}{2}}\left(\tilde{\mathfrak{D}}_{\mathfrak{x}}^{\varepsilon}(x)\right)^{\frac{1}{4}}\right\} .
\end{aligned}
$$

Since $\varepsilon \in(0,1)$, this gives the first stated bound. By Young's inequality, we consequently have

$$
\left.\|x\|_{\infty} \leq \frac{2^{1 / 4}}{\sqrt{\mho_{-}}} \max \left\{1,2^{\frac{1}{4}} \sqrt{\mho_{+}}\right\}\right\}\left\{\frac{3}{2}\left(\tilde{\mathfrak{D}}_{\mathfrak{x}}^{\varepsilon}(x)\right)^{\frac{1}{2}}+\frac{\varepsilon}{2}\right\},
$$

and this gives us the second bound. 
For each $\delta>0$, define

$$
\tilde{\mathcal{B}}_{\mathfrak{x}}^{\varepsilon}(\delta) \stackrel{\text { def }}{=}\left\{x \in \square: \mathfrak{D}_{\mathfrak{x}}^{\varepsilon}(x) \leq \delta\right\} \quad \text { and } \quad \mathcal{B}_{\mathfrak{x}}^{\varepsilon}(\delta) \stackrel{\text { def }}{=} \tilde{\phi}_{\mathfrak{x}}\left(\mathcal{B}_{\mathfrak{x}}^{\varepsilon}(\delta)\right) .
$$

Fix now $\bar{\delta} \in(0,1)$ such that

$$
\bar{\delta}<\left(\frac{\varpi}{2 K_{3.6}}\right)^{4} .
$$

Then if $\delta \in(0, \bar{\delta}), \varepsilon \in(0,1)$, and $\mathfrak{x} \in \mathscr{X}$,

$$
\|x\|_{\infty} \leq K_{3.6}\left\{\delta^{\frac{1}{2}}+\delta^{\frac{1}{4}}\right\}=K_{3.6} \delta^{\frac{1}{4}}\left\{1+\delta^{\frac{1}{4}}\right\} \leq 2 K_{3.6} \delta^{\frac{1}{4}}<\varpi
$$

for all $x \in \tilde{\mathcal{B}}_{\mathfrak{x}}^{\varepsilon}(\delta)$; i.e., $\tilde{\mathcal{B}}_{\mathfrak{x}}(\delta) \Subset \square$.

Let's now put things together. First, we need a cutoff function.

DEFINITION 3.7 (Cutoff Function) Let $\eta \in C_{c}^{\infty}(\mathbb{R} ;[0,1])$ be even and such that $\eta(u)=1$ when $|u| \leq 1$ and $\eta(u)=0$ when $|u| \geq 2$.

For all $\delta \in(0, \bar{\delta}), \varepsilon \in(0,1)$, and $\mathfrak{x} \in \mathscr{X}$, define

$$
\eta_{\mathfrak{x}}^{\delta, \varepsilon}(x) \stackrel{\text { def }}{=} \eta\left(\frac{\mathfrak{D}_{\mathfrak{x}}^{\varepsilon}(x)}{\delta}\right) \chi_{\mathbf{B}_{\mathfrak{x}}}(x), \quad x \in \mathrm{M} ;
$$

then $\eta_{\mathfrak{x}}^{\delta, \varepsilon}$ is smooth and

$$
\operatorname{supp} \eta_{\mathfrak{x}}^{\delta, \varepsilon} \subset \mathcal{B}_{\mathfrak{x}}^{\varepsilon}(2 \delta) \quad \text { and } \quad \operatorname{supp}\left\{1-\sum_{\mathfrak{x} \in \mathscr{X}} \eta_{\mathfrak{x}}^{\delta, \varepsilon}\right\} \subset \mathrm{M} \backslash \bigcup_{\mathfrak{x} \in \mathscr{X}} \mathcal{B}_{\mathfrak{x}}^{\varepsilon}(\delta) .
$$

For all $\delta \in(0, \bar{\delta})$ and $\varepsilon \in(0,1)$, define now

$$
\mathrm{u}^{\delta, \varepsilon}(x) \stackrel{\text { def }}{=}\left\{1-\sum_{\mathfrak{x} \in \mathscr{X}} \eta_{\mathfrak{x}}^{\delta, \varepsilon}(x)\right\} \Phi_{A}^{\varepsilon}(x)+\sum_{\mathfrak{x} \in \mathscr{X}} \eta_{\mathfrak{x}}^{\delta, \varepsilon}(x) \Phi_{B, \mathfrak{x}}^{\varepsilon}(x), \quad x \in \mathrm{M},
$$

and for each $\mathfrak{x} \in \mathscr{X}$, define

$$
\tilde{\mathrm{u}}_{\mathfrak{x}}^{\delta, \varepsilon}(x) \stackrel{\text { def }}{=} \mathrm{u}^{\delta, \varepsilon}\left(\tilde{\phi}_{\mathfrak{x}}(x)\right), \quad x \in \square .
$$

Define

$$
\mathcal{E}(z) \stackrel{\text { def }}{=} \exp \left[-\frac{1}{2} \sqrt{\frac{\pi}{\overline{\mathscr{G}}}} \sqrt{z^{2}+1}\right], \quad z \in \mathbb{R} ;
$$

then $\mathcal{E}$ is smooth and there is a constant $K_{(3.12)}>0$ such that

$$
\frac{1}{K_{(3.12)}} \exp \left[-\frac{1}{2} \sqrt{\frac{\pi}{\bar{G}}}|z|\right] \leq \mathcal{E}(z) \leq K_{(3.12)} \exp \left[-\frac{1}{2} \sqrt{\frac{\pi}{\overline{\mathscr{G}}}}|z|\right]
$$

for all $z \in \mathbb{R}$. The main result of this section is the following: 
THEOREM 3.8 There are positive constants $K_{3.8}$ and $\varkappa_{3.8}$ such that for all $\delta \in$ $(0, \bar{\delta})$ and $\varepsilon \in(0,1)$ such that $\varepsilon / \sqrt{\delta} \leq \varkappa_{3.8}$, and for all $x \in \mathrm{M}$,

$$
\begin{aligned}
\left|\mathrm{u}^{\delta, \varepsilon}(x)\right| \leq & K_{3.8}, \\
\left|\mathscr{L}^{\varepsilon} \mathrm{u}^{\delta, \varepsilon}(x)\right| \leq & \frac{K_{3.8}}{\sqrt{\delta}} \mathcal{E}\left(\frac{\mathrm{H}(x)}{\varepsilon}\right)+K_{3.8} \frac{\|\nabla \mathrm{H}(x)\|^{2}}{\varepsilon} \mathcal{E}\left(\frac{\mathrm{H}(x)}{\varepsilon \sqrt{\delta}}\right) \\
& +\frac{K_{3.8}}{\varepsilon} \exp \left[-\frac{1}{K_{3.8}} \frac{\sqrt{\delta}}{\varepsilon}\right] .
\end{aligned}
$$

We will prove this result at the end of Section 4.

To begin to understand what goes into this theorem, let's explicitly apply $\mathscr{L}^{\varepsilon}$ to $\mathrm{u}^{\delta, \varepsilon}$.

LEMMA 3.9 For all $\delta \in(0, \bar{\delta}), \varepsilon \in(0,1)$, and $x \in \mathrm{M}$,

$$
\mathscr{L}^{\varepsilon} \mathbf{u}^{\delta, \varepsilon}(x)=\sum_{i=1}^{4} \bar{I}_{i}^{\delta, \varepsilon}(x)
$$

where

$$
\begin{aligned}
& \bar{I}_{1}^{\delta, \varepsilon}(x) \stackrel{\text { def }}{=}\left\{1-\sum_{\mathfrak{x} \in \mathscr{X}} \eta_{\mathfrak{x}}^{\delta, \varepsilon}(x)\right\}\left(\mathscr{L}^{\varepsilon} \Psi_{A}^{\varepsilon}\right)(x), \\
& \bar{I}_{2}^{\delta, \varepsilon}(x) \stackrel{\text { def }}{=} \sum_{\mathfrak{x} \in \mathscr{X}}\left(\mathscr{L}^{\varepsilon} \Phi_{B, \mathfrak{x}}^{\varepsilon}\right)(x) \eta_{\mathfrak{x}}^{\delta, \varepsilon}(x), \\
& \bar{I}_{3}^{\delta, \varepsilon}(x)^{\prime} \stackrel{\text { def }}{=} \sum_{\mathfrak{x} \in \mathscr{X}}\left(\Phi_{B, \mathfrak{x}}^{\varepsilon}(x)-\Phi_{A}^{\varepsilon}(x)\right)\left(\mathscr{L}^{\varepsilon} \eta_{\mathfrak{x}}^{\delta, \varepsilon}\right)(x), \\
& \bar{I}_{4}^{\delta, \varepsilon}(x) \stackrel{\text { def }}{=} \sum_{\mathfrak{x} \in \mathscr{X}}\left(\nabla\left(\Phi_{B, \mathfrak{x}}^{\varepsilon}-\Phi_{A}^{\varepsilon}\right), \nabla \eta_{\mathfrak{x}}^{\delta, \varepsilon}(x)\right) .
\end{aligned}
$$

PROOF: The proofs are straightforward calculations.

\section{Calculations and Estimates}

Let's now start bounding things. We need to do the following:

(1) bound $\mathscr{L}^{\varepsilon} \Phi_{A}^{\varepsilon}$ on $\mathrm{M} \backslash \bigcup_{\mathfrak{x} \in \mathscr{X}} \mathcal{B}_{\mathfrak{x}}^{\varepsilon}(\delta)$ (Lemma 4.1),

(2) bound $\mathscr{L}^{\varepsilon} \Phi_{B, \mathfrak{x}}^{\varepsilon}$ on $\mathcal{B}_{\mathfrak{x}}^{\varepsilon}(2 \delta)$ (Lemma 4.3), and

(3) compare $\Phi_{A}^{\varepsilon}$ and $\Phi_{B, \mathfrak{x}}^{\varepsilon}$ (and their first derivatives) on $\mathcal{B}_{\mathfrak{x}}^{\varepsilon}(2 \delta) \backslash \mathcal{B}_{\mathfrak{x}}^{\varepsilon}(\delta)$ (Lemmas 4.4 and 4.7).

LEMMA 4.1 There is a $K>0$ such that if $\delta \in(0, \bar{\delta})$ and $\varepsilon \in(0,1)$,

$$
\left|\mathscr{L}^{\varepsilon} \Phi_{A}^{\varepsilon}(x)\right| \leq \frac{K}{\sqrt{\delta}}\left\{1+\left(\frac{\varepsilon}{\sqrt{\delta}}\right)^{3}\right\} \mathcal{E}\left(\frac{\mathrm{H}(x)}{\varepsilon}\right)
$$

for all $x \in \mathrm{M} \backslash \bigcup_{\mathfrak{x} \in \mathscr{X}} \mathcal{B}_{\mathfrak{x}}^{\varepsilon}(\delta)$. 
Proof: Fix $\ell \in \Lambda$. For $\delta$ and $\varepsilon$ as required and $x \in \mathrm{M}_{\ell} \cap \mathcal{N}$,

$$
\begin{aligned}
& \left(\mathscr{L}^{\varepsilon} \Phi_{A}^{\varepsilon}\right)(x)=\frac{\partial \Psi_{\ell}}{\partial \theta}\left(\neg^{\varepsilon}(x)\right) \frac{\left(\bar{\nabla} \mathrm{H}, \nabla \Theta_{\ell}\right)(x)-\beth\|(x) \nabla \mathrm{H}\|^{2}(x)}{\varepsilon} \\
& +\left\{\frac{\partial \Psi_{\ell}}{\partial h}\left(\neg^{\varepsilon}(x)\right)(\mathscr{L} \mathrm{H})(x)+\beth(x) \frac{\partial^{2} \Psi_{\ell}}{\partial \theta \partial h}\left(\neg^{\varepsilon}(x)\right)\left(\nabla \mathrm{H}, \nabla \Theta_{\ell}\right)(x)\right\} \\
& +\varepsilon\left\{\frac{\partial \Psi_{\ell}}{\partial \theta}\left(\neg^{\varepsilon}(x)\right)\left(\mathscr{L} \Theta_{\ell}\right)(x)+\frac{J(x)}{2} \frac{\partial^{2} \Psi_{\ell}}{\partial \theta^{2}}\left(\neg^{\varepsilon}(x)\right)\left\|\nabla \Theta_{\ell}\right\|^{2}(x)\right\}
\end{aligned}
$$

where $\urcorner^{\varepsilon}(x) \stackrel{\text { def }}{=}\left(\Theta_{\ell}(x), \mathrm{H}(x) / \varepsilon\right)$. Since

$$
\inf \left\{\mathrm{d}_{\ell}\left(\Theta_{\ell}(x)\right)+\left|\frac{\mathrm{H}(x)}{\varepsilon}\right|^{2}: \varepsilon \in(0,1), x \in \mathcal{N} \backslash \bigcup_{\mathfrak{x} \in \mathscr{X}} \mathbf{B}_{\mathfrak{x}}\right\}
$$

is positive, we have the desired bound on $\mathrm{M} \backslash \bigcup_{\mathfrak{x} \in \mathscr{X}} \mathbf{B}_{\mathfrak{x}}$. The only complication is the first term. Here we use Lemma 2.16 and observe that there is a constant $K>0$ such that

$$
\left|\frac{\mathrm{H}}{\varepsilon}\right| \exp \left[-\sqrt{\frac{\pi}{\bar{G}}} \frac{|\mathrm{H}|}{\varepsilon}\right] \leq K \exp \left[-\frac{1}{2} \sqrt{\frac{\pi}{\bar{G}}} \frac{|\mathrm{H}|}{\varepsilon}\right]
$$

on $\mathrm{M}$ for all $\varepsilon \in(0,1)$.

Now fix $\mathfrak{x} \in \mathscr{X} \cap \Gamma_{\ell}$. We want to bound $\tilde{\mathscr{L}}_{\mathfrak{x}}^{\varepsilon} \tilde{\Phi}_{A, \mathfrak{x}}^{\varepsilon}$ in $\tilde{\mathcal{N}}_{\mathfrak{x}} \backslash \tilde{\mathcal{B}}_{\mathfrak{x}}^{\varepsilon}(\delta)$. Define $\tilde{7}^{\varepsilon}(x) \stackrel{\text { def }}{=}\left(\tilde{\Theta}_{\mathfrak{x}}(x)+\tilde{\Theta}_{\mathfrak{x}}^{\oplus}(x), \tilde{\mathrm{H}}(x) / \varepsilon\right)$ for all $\varepsilon \in\left(0, \stackrel{1}{1)}\right.$ and $x \in \tilde{\mathcal{N}}_{\mathfrak{x}}$. There are then positive constants $K$ and $K^{\prime}$ such that

$$
\begin{aligned}
& \left(\mathcal{E}\left(\frac{\mathrm{H}(x)}{\varepsilon}\right)\right)^{-1}\left|\frac{\partial \Psi_{\ell}}{\partial \theta}\left(\tilde{\mathcal{\urcorner}}^{\varepsilon}(x)\right) \frac{\left((\bar{\nabla} \mathrm{H}, \nabla \Theta)-\beth\|\nabla \mathrm{H}\|^{2}\right)\left(\tilde{\phi}_{\mathfrak{x}}(x)\right)}{\varepsilon}\right| \\
& \leq \frac{K|\tilde{\mathrm{H}}(x)|}{\varepsilon \tilde{\mathfrak{D}}_{\mathfrak{x}}^{\varepsilon}(x)}=\frac{K^{\prime} \varepsilon \sqrt{\tilde{\mathfrak{D}}_{\mathfrak{x}}^{\varepsilon}(x)}}{\varepsilon \tilde{\mathfrak{D}}_{\mathfrak{x}}^{\varepsilon}(x)}=\frac{K^{\prime}}{\sqrt{\tilde{\mathfrak{D}}_{\mathfrak{x}}^{\varepsilon}(x)}} \leq \frac{K^{\prime}}{\sqrt{\delta}}, \\
& \left(\mathcal{E}\left(\frac{\mathrm{H}(x)}{\varepsilon}\right)\right)^{-1}\left|\frac{\partial \Psi_{\ell}}{\partial h}\left(\tilde{\overline{\urcorner}}^{\varepsilon}(x)\right)\left(\tilde{\mathscr{L}}_{\mathfrak{x}} \tilde{\mathrm{H}}\right)(x)\right| \leq \frac{K}{\sqrt{\tilde{\mathfrak{D}}_{\mathfrak{x}}^{\varepsilon}(x)}} \leq \frac{K}{\sqrt{\delta}}, \\
& \left(\mathcal{E}\left(\frac{\mathrm{H}(x)}{\varepsilon}\right)\right)^{-1}\left|\frac{\partial^{2} \Psi_{\ell}}{\partial \theta \partial h}\left(\tilde{\neg}^{\varepsilon}(x)\right)\left(\tilde{\nabla}_{\mathfrak{x}} \tilde{\mathrm{H}}, \tilde{\nabla}_{\mathfrak{x}} \tilde{\Theta}_{\mathfrak{x}}\right)_{E}(x)\right| \\
& \leq \frac{K\|x\|_{\infty}^{2}}{\left(\tilde{\mathfrak{D}}_{\mathfrak{x}}^{\varepsilon}(x)\right)^{3 / 2}} \\
& \leq \frac{K^{\prime}\left\{\tilde{\mathfrak{D}}_{\mathfrak{x}}^{\varepsilon}(x)+\varepsilon^{2}\right\}}{\left(\tilde{\mathfrak{D}}_{\mathfrak{x}}^{\varepsilon}(x)\right)^{3 / 2}}=K^{\prime}\left\{\frac{1}{\sqrt{\tilde{\mathfrak{D}}_{\mathfrak{x}}^{\varepsilon}(x)}}+\frac{\varepsilon^{2}}{\left(\tilde{\mathfrak{D}}_{\mathfrak{x}}^{\varepsilon}(x)\right)^{3 / 2}}\right\} \leq K^{\prime}\left\{\frac{1}{\sqrt{\delta}}+\frac{\varepsilon^{2}}{\delta^{3 / 2}}\right\},
\end{aligned}
$$




$$
\begin{aligned}
& \left(\mathcal{E}\left(\frac{\mathrm{H}(x)}{\varepsilon}\right)\right)^{-1} \varepsilon\left|\frac{\partial \Psi_{\ell}}{\partial \theta}\left(\tilde{\nabla}^{\varepsilon}(x)\right)\left(\tilde{\mathscr{L}}_{\mathfrak{x}} \tilde{\Theta}_{\mathfrak{x}}\right)(x)\right| \leq \frac{K \varepsilon}{\tilde{\mathfrak{D}}_{\mathfrak{x}}^{\varepsilon}(x)} \leq \frac{K \varepsilon}{\delta}, \\
& \left(\mathcal{E}\left(\frac{\mathrm{H}(x)}{\varepsilon}\right)\right)^{-1} \varepsilon\left|\frac{\partial^{2} \Psi_{\ell}}{\partial \theta^{2}}\left(\tilde{\mathcal{\nabla}}^{\varepsilon}(x)\right)\left\|\tilde{\nabla}_{\mathfrak{x}} \tilde{\Theta}_{\mathfrak{x}}\right\|_{E}^{2}(x)\right| \\
& \quad \leq \frac{K\|x\|_{\infty}^{2} \varepsilon}{\left(\tilde{\mathfrak{D}}_{\mathfrak{x}}^{\varepsilon}(x)\right)^{2}} \\
& \quad \leq \frac{K^{\prime} \varepsilon\left\{\tilde{\mathfrak{D}}_{\mathfrak{x}}^{\varepsilon}(x)+\varepsilon^{2}\right\}}{\left(\tilde{\mathfrak{D}}_{\mathfrak{x}}^{\varepsilon}(x)\right)^{2}}=K^{\prime}\left\{\frac{\varepsilon}{\tilde{\mathfrak{D}}_{\mathfrak{x}}^{\varepsilon}(x)}+\frac{\varepsilon^{3}}{\left(\tilde{\mathfrak{D}}_{\mathfrak{x}}^{\varepsilon}(x)\right)^{2}}\right\} \leq K^{\prime}\left\{\frac{\varepsilon}{\delta}+\frac{\varepsilon^{3}}{\delta^{2}}\right\},
\end{aligned}
$$

for all $\delta$ and $\varepsilon$ as required and all $x \in \tilde{\mathcal{N}}_{\mathfrak{x}} \backslash \tilde{\mathcal{B}}_{\mathfrak{x}}^{\varepsilon}(\delta)$ such that $\phi_{\mathfrak{x}}(x) \in \mathrm{M}_{\ell}$. Combining things, we get that there is a constant $K>0$ such that

$$
\left|\tilde{\mathscr{L}}_{\mathfrak{x}}^{\varepsilon} \tilde{\Phi}_{A, \mathfrak{x}}^{\varepsilon}(x)\right| \leq \frac{K}{\sqrt{\delta}}\left\{1+\frac{\varepsilon}{\sqrt{\delta}}+\frac{\varepsilon^{2}}{\delta}+\frac{\varepsilon^{3}}{\delta^{3 / 2}}\right\} \mathcal{E}\left(\frac{\mathrm{H}(x)}{\varepsilon}\right)
$$

for all $\delta$ and $\varepsilon$ as required and all $x \in \tilde{\mathcal{N}}_{\mathfrak{x}} \backslash \tilde{\mathcal{B}}_{\mathfrak{x}}^{\varepsilon}(\delta)$ such that $\tilde{\phi}_{\mathfrak{x}}(x) \in \mathrm{M}_{\ell}$. The stated conclusion readily follows from this.

Fix now $\mathfrak{x} \in \mathscr{X}$. We next want to study $\tilde{\mathscr{L}}_{\mathfrak{x}}^{\varepsilon} \tilde{\Phi}_{B, \mathfrak{x}}^{\varepsilon}$. We start with an auxiliary result. For convenience, define $\tilde{\xi}_{\mathfrak{x}}(x) \stackrel{\text { def }}{=} \tilde{\beth}_{\mathfrak{x}}(x) \tilde{\mathrm{B}}_{\mathfrak{x}}(x)\left\|\tilde{\nabla}_{\mathfrak{x}} \tilde{\vartheta}_{\mathfrak{x}, 1}(x)\right\|_{E}^{2}$ for all $x \in \square$.

LEMMA 4.2 We have that

$$
\begin{aligned}
& \left(\bar{\nabla}_{E} \tilde{\mathrm{H}}, \nabla_{E} \tilde{\vartheta}_{\mathfrak{x}, 1}\right)_{E}\left(x_{1}, x_{2}\right)=\frac{1}{2} \tilde{\vartheta}_{\mathfrak{x}, 1}\left(x_{1}, x_{2}\right) \tilde{\xi}_{\mathfrak{x}}\left(0, x_{2}\right), \\
& \left(\bar{\nabla}_{E} \tilde{\mathrm{H}}, \nabla_{E} \tilde{\vartheta}_{\mathfrak{x}, 2}\right)_{E}\left(x_{1}, x_{2}\right)=-\frac{1}{2} \tilde{\vartheta}_{\mathfrak{x}, 2}\left(x_{1}, x_{2}\right) \tilde{\xi}_{\mathfrak{x}}\left(0, x_{2}\right),
\end{aligned}
$$

for all $\left(x_{1}, x_{2}\right) \in \square$. Also, there is a constant $K>0$ such that for $i \in\{1,2\}$ and $x \in \square$,

$$
\left\|\nabla_{E} \tilde{\vartheta}_{\mathfrak{x}, i}(x)\right\|_{E} \leq K \quad \text { and } \quad\left|\tilde{\mathscr{L}}_{\mathfrak{x}} \tilde{\vartheta}_{\mathfrak{x}, i}(x)\right| \leq K
$$

Proof: The stated regularity bounds are fairly obvious. In light of this regularity, it suffices to carry out our calculations on $\tilde{\mathcal{N}}_{\mathfrak{x}}$. For $\left(x_{1}, x_{2}\right) \in \tilde{\mathcal{N}}_{\mathfrak{x}}$,

$$
\begin{aligned}
\left(\bar{\nabla}_{E} \tilde{\mathrm{H}}, \nabla_{E} \tilde{\vartheta}_{\mathfrak{x}, 1}\right)_{E}\left(x_{1}, x_{2}\right) & =\mathfrak{s}\left(x_{2}\right) \frac{\tilde{\mathrm{H}}\left(x_{1}, x_{2}\right)}{2\left(-\tilde{\Theta}_{\mathfrak{x}}\left(0, x_{2}\right)\right)^{3 / 2}}\left\{-x_{2} \frac{\partial \tilde{\Theta}_{\mathfrak{x}}}{\partial x_{2}}\left(0, x_{2}\right)\right\} \\
& =\frac{1}{2} \tilde{\vartheta}_{\mathfrak{x}, 1}\left(x_{1}, x_{2}\right) \frac{\tilde{F}_{\mathfrak{x}}\left(0, x_{2}\right)}{-\tilde{\Theta}_{\mathfrak{x}}\left(x_{1}, x_{2}\right)},
\end{aligned}
$$




$$
\begin{aligned}
\left(\bar{\nabla}_{E} \tilde{\mathrm{H}}, \nabla_{E} \tilde{\vartheta}_{\mathfrak{x}, 2}\right)_{E}\left(x_{1}, x_{2}\right) & =-\mathfrak{s}\left(x_{2}\right) \frac{1}{2 \sqrt{-\tilde{\Theta}_{\mathfrak{x}}\left(0, x_{2}\right)}}\left\{-x_{2} \frac{\partial \tilde{\Theta}_{\mathfrak{x}}}{\partial x_{2}}\left(0, x_{2}\right)\right\} \\
& =-\frac{1}{2} \tilde{\vartheta}_{\mathfrak{x}, 2}\left(x_{1}, x_{2}\right) \frac{\tilde{F}_{\mathfrak{x}}\left(0, x_{2}\right)}{-\tilde{\Theta}_{\mathfrak{x}}\left(x_{1}, x_{2}\right)} .
\end{aligned}
$$

We now calculate that

$$
\tilde{\xi}_{\mathfrak{x}}\left(0, x_{2}\right)=\frac{\tilde{\mathcal{J}}_{\mathfrak{x}}\left(0, x_{2}\right) \tilde{\mathrm{B}}_{\mathfrak{x}}\left(0, x_{2}\right) \tilde{g}_{\mathfrak{x}, 1,1}^{-1}\left(0, x_{2}\right)}{\tilde{\widetilde{S}}_{\mathfrak{x}}^{V}\left(x_{2}\right)}=\frac{\tilde{F}_{\mathfrak{x}}\left(0, x_{2}\right)}{x_{2}^{2} \tilde{\mho}_{\mathfrak{x}}^{V}\left(x_{2}\right)}=\frac{\tilde{F}_{\mathfrak{x}}\left(0, x_{2}\right)}{-\tilde{\Theta}_{\mathfrak{x}}\left(0, x_{2}\right)}
$$

for all $\left(x_{1}, x_{2}\right) \in \square$, and this gives us the claimed PDE for $\tilde{\vartheta}_{\mathfrak{x}, 1}$ and $\tilde{\vartheta}_{\mathfrak{x}, 2}$.

The heart of our analysis of $\tilde{\mathscr{L}}_{\mathfrak{x}}^{\varepsilon} \tilde{\Phi}_{B, \mathfrak{x}}^{\varepsilon}$ is the standard fact that $\mathfrak{g}^{(1)}(z)=-z \mathfrak{g}(z)$ for all $z \in \mathbb{R}$.

LEMMA 4.3 There is a $K>0$ such that for all $\delta \in(0, \bar{\delta})$ and $\varepsilon \in(0,1)$,

$$
\left|\tilde{\mathscr{L}}_{\mathfrak{x}}^{\varepsilon} \tilde{\Phi}_{B, \mathfrak{x}}^{\varepsilon}(x)\right| \leq K\left\{1+\frac{\|x\|_{\infty}^{2}}{\varepsilon}\right\}, \quad x \in \tilde{\mathcal{B}}_{\mathfrak{x}}^{\varepsilon}(2 \delta) .
$$

Proof: Let I be either the interval $\mathbb{R}_{+}$or $\mathbb{R}_{-}$, and let $\varphi$ be a smooth function on I such that $\dot{\varphi}$ and $\ddot{\varphi}$ are bounded. Recalling Proposition 3.2 and the comments preceding Remark 3.4, we know that $u_{\mathfrak{r}, L}$ and $u_{\mathfrak{x}, R}$ fit these requirements. For all $\varepsilon$ and $x$ as required, define the function

$$
\Upsilon^{\varepsilon}(x) \stackrel{\text { def }}{=} \varepsilon \int_{z \in \mathrm{I}} \mathfrak{g}\left(\frac{\tilde{\vartheta}_{\mathfrak{x}, 1}(x)}{\varepsilon}-z\right) \varphi\left(\tilde{\vartheta}_{\mathfrak{x}, 2}(x) z\right) d z .
$$

Fix such an $\varepsilon$ and $x=\left(x_{1}, x_{2}\right)$. Then

$$
\left(\tilde{\mathscr{L}}_{\mathfrak{x}}^{\varepsilon} \Upsilon^{\varepsilon}\right)(x)=\mathrm{E}_{1}^{\varepsilon}(x)+\mathrm{E}_{2}^{\varepsilon}(x)+\mathrm{E}_{3}^{\varepsilon}(x)
$$

where

$$
\begin{aligned}
\mathrm{E}_{1}^{\varepsilon}(x)=\int_{z \in \mathrm{I}}\{ & \mathfrak{g}^{(1)}\left(\frac{\tilde{\vartheta}_{\mathfrak{x}, 1}(x)}{\varepsilon}-z\right) \frac{\left(\bar{\nabla}_{E} \tilde{\mathrm{H}}, \nabla_{E} \tilde{\vartheta}_{\mathfrak{x}, 1}\right)_{E}(x)}{\varepsilon^{2} \tilde{\mathrm{B}}_{\mathfrak{x}}(x)} \varphi\left(\tilde{\vartheta}_{\mathfrak{x}, 2}(x) z\right) \\
& +\mathfrak{g}^{(2)}\left(\frac{\tilde{\vartheta}_{\mathfrak{x}, 1}(x)}{\varepsilon}-z\right) \frac{\tilde{\mathfrak{J}}_{\mathfrak{x}}(x)\left\|\tilde{\nabla}_{\mathfrak{x}} \tilde{\vartheta}_{\mathfrak{x}, 1}(x)\right\|_{E}^{2}}{2 \varepsilon} \varphi\left(\tilde{\vartheta}_{\mathfrak{x}, 2}(x) z\right) \\
& \left.+z \mathfrak{g}\left(\frac{\tilde{\vartheta}_{\mathfrak{x}, 1}(x)}{\varepsilon}-z\right) \dot{\varphi}\left(\tilde{\vartheta}_{\mathfrak{x}, 2}(x) z\right) \frac{\left(\bar{\nabla}_{E} \tilde{\mathrm{H}}, \nabla_{E} \tilde{\vartheta}_{\mathfrak{x}, 2}\right)_{E}(x)}{\varepsilon \tilde{\mathrm{B}}_{\mathfrak{x}}(x)}\right\} d z,
\end{aligned}
$$




$$
\begin{aligned}
& \mathrm{E}_{2}^{\varepsilon}(x)=\int_{z \in \mathrm{I}} \mathfrak{g}^{(1)}\left(\frac{\tilde{\vartheta}_{\mathfrak{x}, 1}(x)}{\varepsilon}-z\right)\{\left(\tilde{\mathscr{L}}_{\mathfrak{x}} \tilde{\vartheta}_{\mathfrak{x}, 1}\right)(x) \varphi\left(\tilde{\vartheta}_{\mathfrak{x}, 2}(x) z\right) \\
&\left.+\tilde{\beth}_{\mathfrak{x}}(x)\left(\tilde{\nabla}_{\mathfrak{x}} \tilde{\vartheta}_{\mathfrak{x}, 1}, \tilde{\nabla}_{\mathfrak{x}} \tilde{\vartheta}_{\mathfrak{x}, 2}\right)_{E}(x) z \dot{\varphi}\left(\tilde{\vartheta}_{\mathfrak{x}, 2}(x) z\right)\right\} d z, \\
& \mathrm{E}_{3}^{\varepsilon}(x)=\varepsilon \int_{z \in \mathrm{I}} \mathfrak{g}\left(\frac{\tilde{\vartheta}_{\mathfrak{x}, 1}(x)}{\varepsilon}-z\right)\left\{\left(\tilde{\mathscr{L}}_{\mathfrak{x}} \tilde{\vartheta}_{\mathfrak{x}, 2}\right)(x) z \dot{\varphi}\left(\tilde{\vartheta}_{\mathfrak{x}, 2}(x) z\right)\right. \\
&\left.+\frac{1}{2} \tilde{\mathcal{I}}_{\mathfrak{x}}(x)\left\|\tilde{\nabla}_{\mathfrak{x}} \tilde{\vartheta}_{\mathfrak{x}, 2}(x)\right\|_{E}^{2} z^{2} \ddot{\varphi}\left(\tilde{\vartheta}_{\mathfrak{x}, 2}(x) z\right)\right\} d z .
\end{aligned}
$$

We can integrate by parts to get that for $i \in\{1,2\}$,

$$
\begin{aligned}
& \int_{z \in \mathrm{I}} \mathfrak{g}^{(i)}\left(\frac{\tilde{\vartheta}_{\mathfrak{x}, 1}(x)}{\varepsilon}-z\right) \varphi\left(\tilde{\vartheta}_{\mathfrak{x}, 2}(x) z\right) d z= \\
& \pm \mathfrak{g}^{(i-1)}\left(\frac{\tilde{\vartheta}_{\mathfrak{x}, 1}(x)}{\varepsilon}\right) \varphi(0)+\tilde{\vartheta}_{\mathfrak{x}, 2}(x) \int_{z \in \mathrm{I}} \mathfrak{g}^{(i-1)}\left(\frac{\tilde{\vartheta}_{\mathfrak{x}, 1}(x)}{\varepsilon}-z\right) \dot{\varphi}\left(\tilde{\vartheta}_{\mathfrak{x}, 2}(x) z\right) d z
\end{aligned}
$$

where we choose + if $\mathrm{I}=\mathbb{R}_{+}$and - if $\mathrm{I}=\mathbb{R}_{-}$. By carefully calculating, one can see that $\mathrm{E}_{1}^{\varepsilon}(x)=\mathrm{E}_{1,1}^{\varepsilon}(x) \pm \mathrm{E}_{1,2}^{\varepsilon}(x)$ where

$$
\begin{aligned}
& \mathrm{E}_{1,1}^{\varepsilon}(x) \stackrel{\text { def }}{=} \frac{1}{2 \varepsilon \tilde{\mathrm{B}}_{\mathfrak{x}}(x)} \int_{z \in \mathrm{I}}\{ \mathfrak{g}^{(1)}\left(\frac{\tilde{\vartheta}_{\mathfrak{x}, 1}(x)}{\varepsilon}-z\right) \tilde{\xi}_{\mathfrak{x}}(x) \\
&\left.+\mathfrak{g}\left(\frac{\tilde{\vartheta}_{\mathfrak{x}, 1}(x)}{\varepsilon}-z\right)\left(\frac{\tilde{\vartheta}_{\mathfrak{x}, 1}(x)}{\varepsilon}-z\right) \tilde{\xi}_{\mathfrak{x}}\left(0, x_{2}\right)\right\} \\
& \times \tilde{\vartheta}_{\mathfrak{x}, 2}(x) \dot{\varphi}\left(\tilde{\vartheta}_{\mathfrak{x}, 2}(x) z\right) d z \\
&=\frac{1}{2 \varepsilon \tilde{\mathrm{B}}_{\mathfrak{x}}(x)} \int_{z \in \mathrm{I}} \mathfrak{g}^{(1)}\left(\frac{\tilde{\vartheta}_{\mathfrak{x}, 1}(x)}{\varepsilon}-z\right)\left\{\tilde{\xi}_{\mathfrak{x}}(x)-\tilde{\xi}_{\mathfrak{x}}\left(0, x_{2}\right)\right\} \tilde{\vartheta}_{\mathfrak{x}, 2}(x) \dot{\varphi}\left(\tilde{\vartheta}_{\mathfrak{x}, 2}(x) z\right) d z
\end{aligned}
$$

and

$$
\begin{aligned}
\mathrm{E}_{1,2}^{\varepsilon}(x) & \stackrel{\text { def }}{=} \frac{1}{2 \varepsilon \tilde{\mathrm{B}}_{\mathfrak{x}}(x)}\left\{\mathfrak{g}\left(\frac{\tilde{\vartheta}_{\mathfrak{x}, 1}(x)}{\varepsilon}\right) \frac{\tilde{\vartheta}_{\mathfrak{x}, 1}(x)}{\varepsilon} \tilde{\xi}_{\mathfrak{x}}\left(0, x_{2}\right)+\mathfrak{g}^{(1)}\left(\frac{\tilde{\vartheta}_{\mathfrak{x}, 1}(x)}{\varepsilon}\right) \tilde{\xi}_{\mathfrak{x}}(x)\right\} \varphi(0) \\
& =\frac{1}{2 \varepsilon \tilde{\mathrm{B}}_{\mathfrak{x}}(x)} \mathfrak{g}^{(1)}\left(\frac{\tilde{\vartheta}_{\mathfrak{x}, 1}(x)}{\varepsilon}\right)\left\{\tilde{\xi}_{\mathfrak{x}}(x)-\tilde{\xi}_{\mathfrak{x}}\left(0, x_{2}\right)\right\} \varphi(0)
\end{aligned}
$$

Note now that there is a constant $K>0$ such that

$$
\left|\tilde{\xi}_{\mathfrak{x}}\left(0, x_{2}\right)-\tilde{\xi}_{\mathfrak{x}}\left(x_{1}, x_{2}\right)\right| \leq K\left|\tilde{\vartheta}_{\mathfrak{x}, 1}\left(x_{1}, x_{2}\right)\right|, \quad\left(x_{1}, x_{2}\right) \in \square
$$


Furthermore, recall that $\dot{\varphi}$ is assumed to be bounded and observe that $\mathfrak{g}^{(1)}$ is integrable. Thus there is a constant $K>0$ such that for all $\varepsilon$ and $x$ as required,

$$
\begin{aligned}
& \left|\mathrm{E}_{1,1}^{\varepsilon}(x)\right| \leq K \frac{\left|\tilde{\vartheta}_{\mathfrak{x}, 1}(x) \tilde{\vartheta}_{\mathfrak{x}, 2}(x)\right|}{\varepsilon}=K \frac{\|x\|_{\infty}^{2}}{\varepsilon}, \\
& \left|\mathrm{E}_{1,2}^{\varepsilon}(x)\right| \leq K\left|\mathfrak{g}^{(1)}\left(\frac{\tilde{\vartheta}_{\mathfrak{x}, 1}(x)}{\varepsilon}\right)\right| \frac{\left|\tilde{\vartheta}_{\mathfrak{x}, 1}(x)\right|}{\varepsilon} \leq K \sup _{z \in \mathbb{R}}\left|z \mathfrak{g}^{(1)}(z)\right| .
\end{aligned}
$$

Let's next bound $\mathrm{E}_{3}^{\varepsilon}$. Change the variable of integration and use the fact that $\dot{\varphi}$ and $\ddot{\varphi}$ are bounded to find positive constants $K$ and $K^{\prime}$ such that for all $\varepsilon$ and $x$ as required

$$
\begin{aligned}
\left|\mathrm{E}_{3}^{\varepsilon}(x)\right| & \leq K \varepsilon \int_{w \in \mathbb{R}} \mathfrak{g}(w)\left\{\left|\frac{\tilde{\vartheta}_{\mathfrak{x}, 1}(x)}{\varepsilon}+w\right|+\left|\frac{\tilde{\vartheta}_{\mathfrak{x}, 1}(x)}{\varepsilon}+w\right|^{2}\right\} d w \\
& \leq K^{\prime} \varepsilon\left\{1+\frac{\left|\tilde{\vartheta}_{\mathfrak{x}, 1}(x)\right|^{2}}{\varepsilon^{2}}\right\} \leq K^{\prime}\left\{1+\frac{1}{\mho_{-}} \frac{\|x\|_{\infty}^{2}}{\varepsilon}\right\} ;
\end{aligned}
$$

one must use Young's inequality and the triangle inequality to get the second line.

To bound $\mathrm{E}_{2}^{\varepsilon}$, we first integrate by parts, as we did in (4.1). For each $\varepsilon \in(0,1)$, we get that $\mathrm{E}_{2}^{\varepsilon}=\mathrm{E}_{2,1}^{\varepsilon} \pm \mathrm{E}_{2,2}^{\varepsilon}$ on $\square$, where we choose + or - as we did in (4.1), and where

$$
\begin{aligned}
& \mathrm{E}_{2,1}^{\varepsilon}(x)=\int_{z \in \mathrm{I}} \mathfrak{g}\left(\frac{\tilde{\vartheta}_{\mathfrak{x}, 1}(x)}{\varepsilon}-z\right)\{\left(\tilde{\mathscr{L}}_{\mathfrak{x}} \tilde{\vartheta}_{\mathfrak{x}, 1}\right)(x) \tilde{\vartheta}_{\mathfrak{x}, 2}(x) \dot{\varphi}\left(\tilde{\vartheta}_{\mathfrak{x}, 2}(x) z\right) \\
&+\tilde{\beth}_{\mathfrak{x}}(x)\left(\tilde{\nabla}_{\mathfrak{x}} \tilde{\vartheta}_{\mathfrak{x}, 1}, \tilde{\nabla}_{\mathfrak{x}} \tilde{\vartheta}_{\mathfrak{x}, 2}\right)_{E}(x) \\
&\left.\times\left\{\dot{\varphi}\left(\tilde{\vartheta}_{\mathfrak{x}, 2}(x) z\right)+z \tilde{\vartheta}_{\mathfrak{x}, 2}(x) \ddot{\varphi}\left(\tilde{\vartheta}_{\mathfrak{x}, 2}(x) z\right)\right\}\right\} d z, \\
& \mathrm{E}_{2,2}^{\varepsilon}(x)=\mathfrak{g}\left(\frac{\tilde{\vartheta}_{\mathfrak{x}, 1}(x)}{\varepsilon}\right)\left(\tilde{\mathscr{L}}_{\mathfrak{x}} \tilde{\vartheta}_{\mathfrak{x}, 1}\right)(x) \varphi(0) .
\end{aligned}
$$

Bounding now as in (4.2), we have that there are positive constants $K$ and $K^{\prime}$ such that for all $\varepsilon$ and $x$ as required,

$$
\begin{aligned}
\left|\mathrm{E}_{2,1}^{\varepsilon}(x)\right| & \leq K \int_{w \in \mathbb{R}}|\mathfrak{g}(w)|\left\{\left|\tilde{\vartheta}_{\mathfrak{x}, 2}(x)\right|+1+\left|\frac{\tilde{\vartheta}_{\mathfrak{x}, 1}(x)}{\varepsilon}+w\right|\left|\tilde{\vartheta}_{\mathfrak{x}, 2}(x)\right|\right\} d w \\
& \leq K^{\prime}\left\{1+\frac{\|x\|_{\infty}^{2}}{\varepsilon}\right\} \\
\left|\mathrm{E}_{2,2}^{\varepsilon}(x)\right| & \leq K
\end{aligned}
$$

the last bound is a direct one. Combine things to get the stated bound. 
Now let's compare the $\tilde{\Phi}_{A, \mathfrak{x}}^{\varepsilon}$ and $\tilde{\Phi}_{B, \mathfrak{x}}^{\varepsilon}$ on $\tilde{\mathcal{B}}_{\mathfrak{x}}^{\varepsilon}(2 \delta) \backslash \tilde{\mathcal{B}}_{\mathfrak{x}}^{\varepsilon}(\delta)$. We first carry out this comparison on $\mathcal{C}_{\mathfrak{x}, L} \cup \mathcal{C}_{\mathfrak{x}, R}$; recall that $u_{\mathfrak{x}, L}$ and $u_{\mathfrak{x}, R}$ are smooth.

LEMmA 4.4 There is a $K>0$ such that for $\varepsilon \in(0,1)$ and $x \in \mathcal{C}_{\mathfrak{x}, L} \cup \mathcal{C}_{\mathfrak{x}, R}$,

$$
\begin{gathered}
\left|\tilde{\Phi}_{B, \mathfrak{x}}^{\varepsilon}(x)-\tilde{\Phi}_{A, \mathfrak{x}}^{\varepsilon}(x)\right| \leq K \varepsilon\|x\|_{\infty}^{2}+K \varepsilon \exp \left[-\frac{1}{K} \frac{\|x\|_{\infty}^{2}}{\varepsilon^{2}}\right], \\
\left\|\nabla_{E}\left(\tilde{\Phi}_{B, \mathfrak{x}}^{\varepsilon}-\tilde{\Phi}_{A, \mathfrak{x}}^{\varepsilon}\right)(x)\right\|_{E} \leq K\|x\|_{\infty}\left\{\varepsilon+\|x\|_{\infty}^{2}\right\}+K \exp \left[-\frac{1}{K} \frac{\|x\|_{\infty}^{2}}{\varepsilon^{2}}\right] .
\end{gathered}
$$

Proof: First note that $\tilde{\vartheta}_{\mathfrak{x}, 1} \tilde{\vartheta}_{\mathfrak{x}, 2} \equiv \tilde{\mathrm{H}}$ on $\square$. Fix $\varepsilon \in(0,1)$. For $s \in\{L, R\}$ and $x=\left(x_{1}, x_{2}\right) \in \mathcal{C}_{\mathfrak{x}, s}$, define

$$
\begin{aligned}
\Upsilon_{1, s}^{\varepsilon}(x) & \stackrel{\text { def }}{=} \varepsilon \int_{z \in \mathbb{R}} \mathfrak{g}\left(\frac{\tilde{\vartheta}_{\mathfrak{x}, 1}(x)}{\varepsilon}-z\right)\left\{u_{\mathfrak{x}, s}^{\circ}\left(\tilde{\vartheta}_{\mathfrak{x}, 2}(x) z\right)-u_{\mathfrak{x}, s}^{\circ}\left(\frac{\tilde{\mathrm{H}}(x)}{\varepsilon}\right)\right\} d z \\
& =\varepsilon \int_{w \in \mathbb{R}} \mathfrak{g}(w)\left\{u_{\mathfrak{x}, s}^{\circ}\left(\frac{\tilde{\mathrm{H}}(x)}{\varepsilon}-\tilde{\vartheta}_{\mathfrak{x}, 2}(x) w\right)-u_{\mathfrak{x}, s}^{\circ}\left(\frac{\tilde{\mathrm{H}}(x)}{\varepsilon}\right)\right\} d w .
\end{aligned}
$$

Define next $\rceil_{\mathfrak{x}}(z) \stackrel{\text { def }}{=} u_{\mathfrak{x}, R}^{\circ}(z)-u_{\mathfrak{x}, L}^{\circ}(z)$ for all $z \in \mathbb{R}$ and then define

$$
\begin{array}{ll}
\left.\Upsilon_{2, R}^{\varepsilon}(x) \stackrel{\text { def }}{=}-\varepsilon \int_{z=-\infty}^{0} \mathfrak{g}\left(\frac{\tilde{\vartheta}_{\mathfrak{x}, 1}(x)}{\varepsilon}-z\right)\right\urcorner_{\mathfrak{x}}\left(\tilde{\vartheta}_{\mathfrak{x}, 2}(x) z\right) d z, & x \in \mathcal{C}_{\mathfrak{x}, R}, \\
\left.\Upsilon_{2, L}^{\varepsilon}(x) \stackrel{\text { def }}{=} \varepsilon \int_{z=0}^{\infty} \mathfrak{g}\left(\frac{\tilde{\vartheta}_{\mathfrak{x}, 1}(x)}{\varepsilon}-z\right)\right\urcorner_{\mathfrak{x}}\left(\tilde{\vartheta}_{\mathfrak{x}, 2}(x) z\right) d z, & x \in \mathcal{C}_{\mathfrak{x}, L} .
\end{array}
$$

Finally, define for $x=\left(x_{1}, x_{2}\right) \in \mathcal{C}_{\mathfrak{x}, s}$

$$
\Upsilon_{3, s}^{\varepsilon}(x) \stackrel{\text { def }}{=} \varepsilon\left\{u_{\mathfrak{x}, s}^{\circ}\left(\frac{\tilde{\mathrm{H}}(x)}{\varepsilon}\right)-u_{\mathfrak{x}, s}\left(-\tilde{\Theta}_{\mathfrak{x}}\left(x_{1}, 0\right), \frac{\tilde{\mathrm{H}}(x)}{\varepsilon}\right)\right\} .
$$

Fix now $s \in\{L, R\}$. We then have that $\tilde{\Phi}_{B, \mathfrak{x}}^{\varepsilon}-\tilde{\Phi}_{A, \mathfrak{x}}^{\varepsilon}=\sum_{i=1}^{3} \Upsilon_{i, s}^{\varepsilon}$ on $\mathcal{C}_{\mathfrak{x}, s}$ (remember to add and subtract $\tilde{\digamma}_{\mathfrak{x}}$ in carrying out this calculation).

Let's first consider $\Upsilon_{3, s}^{\varepsilon}$, and note that $u_{\mathfrak{x}, R}$ and $u_{\mathfrak{x}, L}$ are smooth. We can compute that for all $\varepsilon \in(0,1)$ and $x \in \mathcal{C}_{\mathfrak{x}, s}$,

$$
\begin{aligned}
\nabla_{E} \Upsilon_{3, s}^{\varepsilon}(x)= & \varepsilon \frac{\partial u_{\mathfrak{x}, s}}{\partial \theta}\left(-\tilde{\Theta}_{\mathfrak{x}}(x), \frac{\tilde{\mathrm{H}}(x)}{\varepsilon}\right) \nabla_{E} \tilde{\Theta}_{\mathfrak{x}}^{B}(x) \\
& +\left\{\frac{\partial u_{\mathfrak{x}, s}}{\partial h}\left(-\tilde{\Theta}_{\mathfrak{x}}(x), \frac{\tilde{\mathrm{H}}(x)}{\varepsilon}\right)-\dot{u}_{\mathfrak{x}, s}^{\circ}\left(\frac{\tilde{\mathrm{H}}(x)}{\varepsilon}\right)\right\} \nabla_{E} \tilde{\mathrm{H}}(x) .
\end{aligned}
$$


It is now fairly easy to find positive constants $K$ and $K^{\prime}$ such that

$$
\begin{aligned}
\left|\Upsilon_{3, s}^{\varepsilon}(x)\right| & \leq K \varepsilon\left|\tilde{\Theta}_{\mathfrak{x}}(x)\right| \leq K^{\prime} \varepsilon\|x\|_{\infty}^{2} \\
\left\|\nabla_{E} \Upsilon_{3, s}^{\varepsilon}(x)\right\| & \leq K\left\{\varepsilon\|x\|_{\infty}+\left|\tilde{\Theta}_{\mathfrak{x}}(x)\right|\left\|\nabla_{E} \tilde{\mathrm{H}}(x)\right\|_{E}\right\} \\
& \leq K^{\prime}\left\{\varepsilon\|x\|_{\infty}+\|x\|_{\infty}^{3}\right\} .
\end{aligned}
$$

Let's next consider $\Upsilon_{1, s}^{\varepsilon}$. Define

$$
\mathrm{E}_{S}(z, h) \stackrel{\text { def }}{=} u_{\mathfrak{x}, s}^{\circ}(h+z)-u_{\mathfrak{x}, s}^{\circ}(h)-\dot{u}_{\mathfrak{x}, s}^{\circ}(h) z=z^{2} \int_{s=0}^{1}(1-s) \ddot{u}_{\mathfrak{x}, s}^{\circ}(h+s z) d s
$$

for all $z$ and $h$ in $\mathbb{R}$. Then there is a $K>0$ such that

$$
\left|\mathrm{E}_{s}(z, h)\right| \leq K z^{2}, \quad\left|\frac{\partial \mathrm{E}_{s}}{\partial z}(z, h)\right| \leq K|z|, \quad \text { and } \quad\left|\frac{\partial \mathrm{E}_{s}}{\partial h}(z, h)\right| \leq K|z|^{2},
$$

for all $z$ and $h$ in $\mathbb{R}$. Thus for all $\varepsilon \in(0,1)$ and $x \in \mathcal{C}_{\mathfrak{x}, s}$,

$$
\begin{aligned}
\Upsilon_{1, s}^{\varepsilon}(x)= & \varepsilon \int_{w \in \mathbb{R}} \mathfrak{g}(w)\left\{-\dot{u}_{\mathfrak{x}, s}^{\circ}\left(\frac{\tilde{\mathrm{H}}(x)}{\varepsilon}\right) \tilde{\vartheta}_{\mathfrak{x}, 2}(x) w+\mathrm{E}_{S}\left(-w \tilde{\vartheta}_{\mathfrak{x}, 2}(x), \frac{\tilde{\mathrm{H}}(x)}{\varepsilon}\right)\right\} d w \\
= & \varepsilon \int_{w \in \mathbb{R}} \mathfrak{g}(w) \mathrm{E}_{s}\left(-w \tilde{\vartheta}_{\mathfrak{x}, 2}(x), \frac{\tilde{\mathrm{H}}(x)}{\varepsilon}\right) d w \\
\nabla_{E} \Upsilon_{1, s}^{\varepsilon}(x)= & \int_{w \in \mathbb{R}} \mathfrak{g}(w)\left\{-\varepsilon w \frac{\partial \mathrm{E}_{s}}{\partial z}\left(-w \tilde{\vartheta}_{\mathfrak{x}, 2}(x), \frac{\tilde{\mathrm{H}}(x)}{\varepsilon}\right) \nabla_{E} \tilde{\vartheta}_{\mathfrak{x}, 2}(x)\right. \\
& \left.+\frac{\partial \mathrm{E}_{s}}{\partial h}\left(-w \tilde{\vartheta}_{\mathfrak{x}, 2}(x), \frac{\tilde{\mathrm{H}}(x)}{\varepsilon}\right) \nabla_{E} \tilde{\mathrm{H}}(x)\right\} d w
\end{aligned}
$$

where we have used the fact that $\mathfrak{g}$ is even. It is now fairly easy to find positive constants $K$ and $K^{\prime}$ such that for all $\varepsilon \in(0,1)$ and $x \in \mathcal{C}_{\mathfrak{x}, s}$

$$
\begin{aligned}
\left|\Upsilon_{1, s}^{\varepsilon}(x)\right| & \leq K \varepsilon\left|\tilde{\vartheta}_{\mathfrak{x}, 2}(x)\right|^{2} \leq K^{\prime} \varepsilon\|x\|^{2} \\
\left\|\nabla_{E} \Upsilon_{1, s}^{\varepsilon}(x)\right\| & \leq K\left\{\varepsilon\left|\tilde{\vartheta}_{\mathfrak{x}, 2}(x)\right|+\left|\tilde{\vartheta}_{\mathfrak{x}, 2}(x)\right|^{2}\left\|\nabla_{E} \tilde{\mathrm{H}}(x)\right\|\right\} \\
& \leq K^{\prime}\left\{\varepsilon\|x\|_{\infty}^{2}+\|x\|_{\infty}^{3}\right\} .
\end{aligned}
$$

Finally, let's consider $\Upsilon_{2, s}^{\varepsilon}$. To do so efficiently, for each $\varepsilon \in(0,1)$ and $x=$ $\left(x_{1}, x_{2}\right) \in \square$, define

$$
\left.\hat{\Upsilon}_{\mathfrak{x}}^{\varepsilon}(x) \stackrel{\text { def }}{=}-\varepsilon \int_{z=-\infty}^{0} \mathfrak{g}\left(-\frac{\left|\tilde{\vartheta}_{\mathfrak{x}, 1}(x)\right|}{\varepsilon}+z\right)\right\rceil_{\mathfrak{x}}\left(\mathfrak{s}\left(x_{1}\right) \tilde{\vartheta}_{\mathfrak{x}, 2}(x) z\right) d z
$$


then (keeping in mind that $\mathfrak{g}$ is even) $\hat{\Upsilon}_{\mathfrak{x}}^{\varepsilon}=\Upsilon_{2, R}^{\varepsilon}$ on $\mathcal{C}_{\mathfrak{x}, R}$ and $\hat{\Upsilon}_{\mathfrak{x}}^{\varepsilon}=\Upsilon_{2, L}^{\varepsilon}$ on $\mathcal{C}_{\mathfrak{x}, R}$. Integrating by parts and then differentiating, we get that

$$
\begin{aligned}
& \left.\hat{\Upsilon}_{\mathfrak{x}}^{\varepsilon}(x)=-\varepsilon \mathfrak{g}^{(-1)}\left(-\frac{\left|\tilde{\vartheta}_{\mathfrak{x}, 1}(x)\right|}{\varepsilon}\right)\right\rceil_{\mathfrak{x}}(0) \\
& +\varepsilon \mathfrak{s}\left(x_{1}\right) \tilde{\vartheta}_{\mathfrak{x}, 2}(x) \int_{z=-\infty}^{0} \mathfrak{g}^{(-1)}\left(-\frac{\left|\tilde{\vartheta}_{\mathfrak{x}, 1}(x)\right|}{\varepsilon}+z\right) \dot{\boldsymbol{\succ}}_{\mathfrak{x}}\left(\mathfrak{s}\left(x_{1}\right) \tilde{\vartheta}_{\mathfrak{x}, 2}(x) z\right) d z \\
& \left.=-\varepsilon \mathfrak{g}^{(-1)}\left(-\frac{\left|\tilde{\vartheta}_{\mathfrak{x}, 1}(x)\right|}{\varepsilon}\right)\right\urcorner_{\mathfrak{x}}(0) \\
& +\varepsilon \mathfrak{s}\left(x_{1}\right) \tilde{\vartheta}_{\mathfrak{x}, 2}(x) \int_{z=-\infty}^{-\left|\tilde{\vartheta}_{\mathfrak{x}, 1}(x)\right| / \varepsilon} \mathfrak{g}^{(-1)}(w) \dot{\nearrow}_{\mathfrak{x}}\left(\mathfrak{s}\left(x_{1}\right) \tilde{\vartheta}_{\mathfrak{x}, 2}(x) w+\frac{\tilde{\mathrm{H}}(x)}{\varepsilon}\right) d w, \\
& \left.\nabla_{E} \hat{\Upsilon}_{\mathfrak{x}}^{\varepsilon}(x)=\mathfrak{s}\left(x_{1}\right) \mathfrak{g}\left(-\frac{\left|\tilde{\vartheta}_{\mathfrak{x}, 1}(x)\right|}{\varepsilon}\right)\right\rceil_{\mathfrak{x}}(0) \nabla_{E} \tilde{\vartheta}_{\mathfrak{x}, 1}(x)
\end{aligned}
$$

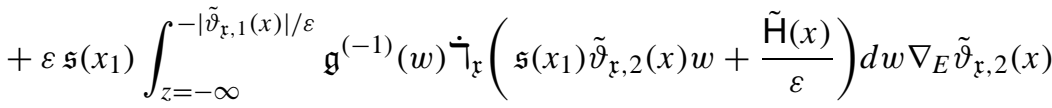

$$
\begin{aligned}
& \left.-\tilde{\vartheta}_{\mathfrak{x}, 2}(x) \mathfrak{g}^{(-1)}\left(-\frac{\left|\tilde{\vartheta}_{\mathfrak{x}, 1}(x)\right|}{\varepsilon}\right)\right\rceil_{\mathfrak{x}}(0) \nabla_{E} \tilde{\vartheta}_{\mathfrak{x}, 1}(x) \\
& +\mathfrak{s}\left(x_{1}\right) \tilde{\vartheta}_{\mathfrak{x}, 2}(x) \int_{z=-\infty}^{-\left|\tilde{\vartheta}_{\mathfrak{x}, 1}(x)\right| / \varepsilon} \mathfrak{g}^{(-1)}(w) \ddot{ך}_{\mathfrak{x}}\left(\mathfrak{s}\left(x_{1}\right) \tilde{\vartheta}_{\mathfrak{x}, 2}(x) w+\frac{\tilde{\mathrm{H}}(x)}{\varepsilon}\right) \\
& \times\left\{\varepsilon \mathfrak{s}\left(x_{1}\right) w \nabla_{E} \tilde{\vartheta}_{\mathfrak{x}, 2}(x)+\nabla_{E} \tilde{\mathrm{H}}(x)\right\} d w,
\end{aligned}
$$

for all $\varepsilon \in(0,1)$ and $x=\left(x_{1}, x_{2}\right) \in \mathcal{C}_{\mathfrak{x}, s}$ (note that then $\left.\mathfrak{s}\left(\tilde{\vartheta}_{\mathfrak{x}, 1}\left(x_{1}, x_{2}\right)\right)=\mathfrak{s}\left(x_{1}\right)\right)$.

We now use the fact that the functions $z \mapsto \mathfrak{g}^{(-1)}(z) e^{z^{2} / 2}$ and $z \mapsto \mathfrak{g}^{(-2)}(z) e^{z^{2} / 2}$ are bounded on $(-\infty, 0)$. There is thus a constant $K>0$ such that for all $\varepsilon$ and $x$ as required,

$$
\begin{aligned}
\left|\hat{\Upsilon}_{\mathfrak{x}}^{\varepsilon}(x)\right| & \leq K \varepsilon \exp \left[-\frac{1}{K} \frac{\left|\tilde{\vartheta}_{\mathfrak{x}, 1}(x)\right|^{2}}{\varepsilon^{2}}\right], \\
\left\|\nabla_{E} \hat{\Upsilon}_{\mathfrak{x}}^{\varepsilon}(x)\right\| & \leq K \exp \left[-\frac{1}{K} \frac{\left|\tilde{\vartheta}_{\mathfrak{x}, 1}(x)\right|^{2}}{\varepsilon^{2}}\right] .
\end{aligned}
$$

To finish, we note that for $x=\left(x_{1}, x_{2}\right) \in \mathcal{C}_{\mathfrak{x}, L} \cup \mathcal{C}_{\mathfrak{x}, R}$,

$$
\|x\|_{\infty} \leq \max \left\{1, \frac{\mho_{+}}{\mho_{-}}\right\}\left|x_{1}\right| \leq \max \left\{1, \frac{\mho_{+}}{\mho_{-}}\right\} \sqrt{\mho_{+}}\left|\tilde{\vartheta}_{\mathfrak{x}, 1}\left(x_{1}\right)\right|
$$


Now let's carry out a similar comparison of $\tilde{\Phi}_{A, \mathfrak{x}}^{\varepsilon}$ and $\tilde{\Phi}_{B, \mathfrak{x}}^{\varepsilon}$ on $\mathcal{C}_{\mathfrak{x}, U} \cup \mathcal{C}_{\mathfrak{x}, D}$. Define for convenience $\tilde{\Theta}_{\mathfrak{x}}^{C}\left(x_{1}, x_{2}\right) \stackrel{\text { def }}{=} \tilde{\Theta}_{\mathfrak{x}}\left(0, x_{2}\right)$ for all $\left(x_{1}, x_{2}\right) \in \square$; then for any $\varepsilon \in(0,1)$,

$$
\tilde{\Phi}_{B, \mathfrak{x}}^{\varepsilon}(x)=\varepsilon \sum_{\ell \in \Lambda} \Psi_{\ell}\left(\tilde{\Theta}_{\mathfrak{x}}^{C}(x)+\tilde{\Theta}_{\mathfrak{x}}^{\oplus}, \frac{\mathrm{H}(x)}{\varepsilon}\right) \chi_{\mathrm{M}_{\ell}}\left(\tilde{\phi}_{\mathfrak{x}}(x)\right), \quad x \in \square .
$$

LEMMA 4.5 There is a constant $K_{4.5}>0$ such that for all $\mathfrak{x} \in \mathscr{X}$ and $x \in \square$,

$$
\left|\tilde{\Theta}_{\mathfrak{x}}^{C}(x)-\tilde{\Theta}_{\mathfrak{x}}(x)\right| \leq K_{4.5}|\tilde{\mathrm{H}}(x)| \text { and }\left\|\nabla_{E} \tilde{\Theta}_{\mathfrak{x}}^{C}(x)\right\|_{E} \leq K_{4.5}\|x\|_{\infty} \text {. }
$$

PROOF: The proof is clear from the explicit formula for $\tilde{\Theta}_{\mathfrak{x}}$.

We also have the following:

LeMma 4.6 For $\delta \in(0, \bar{\delta}), \varepsilon \in(0,1), \mathfrak{x} \in \mathscr{X}$, and $x \in \square$,

$$
\inf _{\lambda \in[0,1]}\left|\lambda \tilde{\Theta}_{\mathfrak{x}}(x)+(1-\lambda) \tilde{\Theta}_{\mathfrak{x}}^{C}(x)\right|+\left|\frac{\tilde{H}(x)}{\varepsilon}\right|^{2} \geq \frac{1}{\sqrt{2}} \tilde{\mathfrak{D}}_{\mathfrak{x}}^{\varepsilon}(x)\left\{1-\sqrt{2} K_{4.5} \frac{\varepsilon}{\sqrt{\tilde{\mathfrak{D}}_{\mathfrak{x}}^{\varepsilon}(x)}}\right\} .
$$

PROOF: For $\delta, \varepsilon, \mathfrak{x}$, and $x$ as required and $\lambda \in[0,1]$,

$$
\begin{aligned}
& \left|\lambda \tilde{\Theta}_{\mathfrak{x}}(x)+(1-\lambda) \tilde{\Theta}_{\mathfrak{x}}^{C}(x)\right|+\left|\frac{\tilde{H}(x)}{\varepsilon}\right|^{2} \\
& \quad \geq\left|\tilde{\Theta}_{\mathfrak{x}}(x)\right|+\left|\frac{\tilde{\mathrm{H}}(x)}{\varepsilon}\right|^{2}-(1-\lambda)\left|\tilde{\Theta}_{\mathfrak{x}}(x)-\tilde{\Theta}_{\mathfrak{x}}^{C}(x)\right| \\
& \quad \geq 2^{-\frac{1}{2}} \tilde{\mathfrak{D}}_{\mathfrak{x}}^{\varepsilon}(x)-K_{4.5}|\tilde{\mathrm{H}}(x)| \\
& \quad \geq 2^{-\frac{1}{2}} \tilde{\mathfrak{D}}_{\mathfrak{x}}^{\varepsilon}(x)-\varepsilon K_{4.5} \sqrt{\tilde{\mathfrak{D}}_{\mathfrak{x}}^{\varepsilon}(x)} .
\end{aligned}
$$

We then have the following:

LEMMA 4.7 There is a $K>0$ such that for all $\delta \in(0, \bar{\delta})$ and $\varepsilon \in(0,1)$ such that $\varepsilon / \sqrt{\delta}<1 /\left(2^{3 / 2} K_{4.5}\right)$, and for all $x \in \tilde{\mathcal{B}}_{\mathfrak{x}}^{\varepsilon}(2 \delta) \backslash \tilde{\mathcal{B}}_{\mathfrak{x}}^{\varepsilon}(\delta)$ such that $x \in \mathcal{C}_{\mathfrak{x}, U} \cup \mathcal{C}_{\mathfrak{x}, D}$,

$$
\begin{aligned}
\left|\tilde{\Phi}_{A, \mathfrak{x}}^{\varepsilon}(x)-\tilde{\Phi}_{B, \mathfrak{x}}^{\varepsilon}(x)\right| & \leq \frac{K \varepsilon^{2}}{\sqrt{\delta}}, \\
\left\|\nabla_{E}\left(\tilde{\Phi}_{A, \mathfrak{x}}^{\varepsilon}-\tilde{\Phi}_{B, \mathfrak{x}}^{\varepsilon}\right)(x)\right\|_{E} & \leq \frac{K \varepsilon\|x\|_{\infty}}{\delta} .
\end{aligned}
$$

Proof: Fix $\ell \in \Lambda$ such that $\mathfrak{x} \in \Gamma_{\ell}$. For any $\varepsilon \in(0,1)$ and $x \in \tilde{\mathcal{N}}_{\mathfrak{x}}$ such that $x \in \mathcal{C}_{\mathfrak{x}, U} \cup \mathcal{C}_{\mathfrak{x}, D}$ and such that $\tilde{\phi}_{\mathfrak{x}}(x) \in \mathrm{M}_{\ell}$,

$$
\begin{aligned}
&\left|\tilde{\Phi}_{A, \mathfrak{x}}^{\varepsilon}(x)-\tilde{\Phi}_{B, \mathfrak{x}}^{\varepsilon}(x)\right| \leq J_{1}^{\varepsilon}(x), \\
&\left\|\nabla_{E}\left(\tilde{\Phi}_{A, \mathfrak{x}}^{\varepsilon}-\tilde{\Phi}_{B, \mathfrak{x}}^{\varepsilon}\right)(x)\right\|_{E} \leq J_{2}^{\varepsilon}(x)+J_{3}^{\varepsilon}(x)+J_{4}^{\varepsilon}(x),
\end{aligned}
$$


where

$$
\begin{aligned}
& J_{1}^{\varepsilon}(x) \stackrel{\text { def }}{=}\left|\Psi_{\ell}\left(\tilde{\Theta}_{\mathfrak{x}}(x)+\tilde{\Theta}_{\mathfrak{x}}^{\oplus}(x), \frac{\tilde{\mathrm{H}}(x)}{\varepsilon}\right)-\Psi_{\ell}\left(\tilde{\Theta}_{\mathfrak{x}}^{C}(x)+\tilde{\Theta}_{\mathfrak{x}}^{\oplus}(x), \frac{\tilde{\mathrm{H}}(x)}{\varepsilon}\right)\right|, \\
& J_{2}^{\varepsilon}(x) \stackrel{\text { def }}{=} \mid \frac{\partial \Psi_{\ell}}{\partial h}\left(\tilde{\Theta}_{\mathfrak{x}}(x)+\tilde{\Theta}_{\mathfrak{x}}^{\oplus}(x), \frac{\tilde{\mathrm{H}}(x)}{\varepsilon}\right) \\
&-\frac{\partial \Psi_{\ell}}{\partial h}\left(\tilde{\Theta}_{\mathfrak{x}}^{C}(x)+\tilde{\Theta}_{\mathfrak{x}}^{\oplus}(x), \frac{\tilde{\mathrm{H}}(x)}{\varepsilon}\right) \mid\left\|\nabla_{E} \tilde{\mathrm{H}}(x)\right\|_{E}, \\
& J_{3}^{\varepsilon}(x) \stackrel{\text { def }}{=} \varepsilon\left|\frac{\partial \Psi_{\ell}}{\partial \theta}\left(\tilde{\Theta}_{\mathfrak{x}}(x)+\tilde{\Theta}_{\mathfrak{x}}^{\oplus}(x), \frac{\tilde{\mathrm{H}}(x)}{\varepsilon}\right)\right|\left\|\nabla_{E} \tilde{\Theta}_{\mathfrak{x}}(x)\right\|_{E}, \\
& J_{4}^{\varepsilon}(x) \stackrel{\text { def }}{=} \varepsilon\left|\frac{\partial \Psi_{\ell}}{\partial \theta}\left(\tilde{\Theta}_{\mathfrak{x}}^{C}(x)+\tilde{\Theta}_{\mathfrak{x}}^{\oplus}(x), \frac{\tilde{H}(x)}{\varepsilon}\right)\right|\left\|\nabla_{E} \tilde{\Theta}_{\mathfrak{x}}^{C}(x)\right\|_{E} .
\end{aligned}
$$

For any $\delta, \varepsilon$, and $x$ as required,

$$
\inf _{\lambda \in[0,1]}\left|\lambda \tilde{\Theta}_{\mathfrak{x}}(x)+(1-\lambda) \tilde{\Theta}_{\mathfrak{x}}^{C}(x)\right|+\left|\frac{\tilde{\mathrm{H}}(x)}{\varepsilon}\right|^{2} \geq \frac{1}{2^{3 / 2}} \tilde{\mathfrak{D}}_{\mathfrak{x}}^{\varepsilon}(x) .
$$

We can now find a positive constant $K$ such that for $i \in\{3,4\}$ and $\delta, \varepsilon$, and $x$ as required,

$$
J_{i}^{\varepsilon}(x) \leq \frac{K \varepsilon\|x\|_{\infty}}{\tilde{\mathfrak{D}}_{\mathfrak{x}}^{\varepsilon}(x)} \leq \frac{K \varepsilon\|x\|_{\infty}}{\delta} .
$$

Consider next $J_{1}^{\varepsilon}$ and $J_{2}^{\varepsilon}$. Fix $j \in\{0,1\}$. For $\varepsilon \in(0,1)$ and $x \in \tilde{\mathcal{N}}_{\mathfrak{x}}$ such that $\tilde{\phi}_{\mathfrak{x}}(x) \in \mathrm{M}_{\ell}$, we have that

$$
\begin{aligned}
& \frac{\partial \Psi_{\ell}}{\partial h^{j}}\left(\tilde{\Theta}_{\mathfrak{x}}(x)+\tilde{\Theta}_{\mathfrak{x}}^{\oplus}(x), \frac{\tilde{\mathrm{H}}(x)}{\varepsilon}\right)-\frac{\partial \Psi_{\ell}}{\partial h^{j}}\left(\tilde{\Theta}_{\mathfrak{x}}^{C}(x)+\tilde{\Theta}_{\mathfrak{x}}^{\oplus}(x), \frac{\tilde{\mathrm{H}}(x)}{\varepsilon}\right)= \\
& \int_{s=0}^{1} \frac{\partial^{j+1} \hat{\Psi}_{\ell}}{\partial h^{j} \partial \theta}\left(t \tilde{\Theta}_{\mathfrak{x}}(x)+(1-t) \tilde{\Theta}_{\mathfrak{x}}^{C}(x)+\tilde{\Theta}_{\mathfrak{x}}^{\oplus}(x), \frac{\tilde{\mathrm{H}}(x)}{\varepsilon}\right) d t\left(\tilde{\Theta}_{\mathfrak{x}}(x)-\tilde{\Theta}_{\mathfrak{x}}^{C}(x)\right) .
\end{aligned}
$$

We now use Lemmas 4.5 and 4.6. For all $\varepsilon \in(0,1), x \in \square$, and $t \in[0,1]$,

$$
\frac{|\tilde{\mathrm{H}}(x)|}{\left(\tilde{\mathfrak{D}}_{\mathfrak{x}}^{\varepsilon}(x)\right)^{j / 2+1}} \leq \frac{K_{3.6} \varepsilon \sqrt{\tilde{\mathfrak{D}}_{\mathfrak{x}}^{\varepsilon}(x)}}{\left(\tilde{\mathfrak{D}}_{\mathfrak{x}}^{\varepsilon}(x)\right)^{j / 2+1}} \leq \frac{K_{3.6} \varepsilon}{\left(\tilde{\mathfrak{D}}_{\mathfrak{x}}^{\varepsilon}(x)\right)^{(j+1) / 2}} .
$$

Thus there is a constant $K>0$ such that for all $\delta, \varepsilon$, and $x$ as required,

$$
J_{1}^{\varepsilon}(x) \leq \frac{K \varepsilon^{2}}{\sqrt{\tilde{\mathfrak{D}}_{\mathfrak{x}}^{\varepsilon}(x)}} \leq \frac{K \varepsilon^{2}}{\sqrt{\delta}} \quad \text { and } \quad J_{2}^{\varepsilon}(x) \leq \frac{K \varepsilon\|x\|_{\infty}}{\tilde{\mathfrak{D}}_{\mathfrak{x}}^{\varepsilon}(x)} \leq \frac{K \varepsilon\|x\|_{\infty}}{\delta}
$$

We can now bound the various terms of Lemma 3.9. Some final estimates are needed, however. 
LEMMA 4.8 There is a constant $K_{4.8}>0$ such that for all $\varepsilon \in(0,1), \mathfrak{x} \in \mathscr{X}$, and $x \in \square$,

$$
\frac{\|x\|_{\infty}^{2}}{\varepsilon^{2}} \geq \frac{1}{K_{4.8}} \min \left\{\frac{\sqrt{\tilde{\mathfrak{D}}_{\mathfrak{x}}^{\varepsilon}(x)}}{\varepsilon}, \frac{\tilde{\mathfrak{D}}_{\mathfrak{x}}^{\varepsilon}(x)}{\varepsilon^{2}}\right\}
$$

ProOF: From the explicit formulae for $\tilde{\mathfrak{D}}_{\mathfrak{x}}^{\varepsilon}$, $\tilde{\Theta}_{\mathfrak{x}}$, and $\tilde{\mathrm{H}}$, there are two positive constants $K$ and $K^{\prime}$ such that for all $\varepsilon, \mathfrak{x}$, and $x$ as required,

$$
\tilde{\mathfrak{D}}_{\mathfrak{x}}^{\varepsilon}(x) \leq K\left\{\left|\tilde{\Theta}_{\mathfrak{x}}(x)\right|+\left|\frac{\tilde{\mathrm{H}}(x)}{\varepsilon}\right|^{2}\right\} \leq K^{\prime}\left\{\|x\|_{\infty}^{2}+\frac{\|x\|_{\infty}^{4}}{\varepsilon^{2}}\right\} ;
$$

thus, either $K^{\prime}\|x\|_{\infty}^{2} \geq \tilde{\mathfrak{D}}_{\mathfrak{x}}^{\varepsilon}(x) / 2$, in which case

$$
\frac{\|x\|_{\infty}^{2}}{\varepsilon^{2}} \geq \frac{1}{2 K^{\prime}} \frac{\tilde{\mathfrak{D}}_{\mathfrak{x}}^{\varepsilon}(x)}{\varepsilon^{2}},
$$

or $K^{\prime}\|x\|^{4} / \varepsilon^{2} \geq \tilde{\mathfrak{D}}_{\mathfrak{x}}^{\varepsilon}(x) / 2$, in which case

$$
\frac{\|x\|_{\infty}^{2}}{\varepsilon^{2}} \geq \frac{1}{\sqrt{2 K^{\prime}}} \frac{\sqrt{\tilde{\mathfrak{D}}_{\mathfrak{x}}^{\varepsilon}(x)}}{\varepsilon}
$$

We also have the following lemma:

LEMMA 4.9 There is a constant $K>0$ such that for all $\delta \in(0, \bar{\delta}), \varepsilon \in(0,1)$, and $x \in \mathrm{M}$,

$$
\begin{aligned}
\left\|\nabla \eta_{\mathfrak{x}}^{\delta, \varepsilon}(x)\right\| & \leq \frac{K}{\varepsilon}\left\{1+\left(\frac{\varepsilon}{\sqrt{\delta}}\right)^{2}\right\} \chi_{\mathcal{B}_{\mathfrak{x}}^{\varepsilon}(2 \delta)}(x), \\
\left|\left(\mathscr{L}^{\varepsilon} \eta_{\mathfrak{x}}^{\delta, \varepsilon}\right)(x)\right| & \leq \frac{K}{\varepsilon^{2}}\left\{1+\left(\frac{\varepsilon}{\sqrt{\delta}}\right)^{4}\right\} \chi_{\mathcal{B}_{\mathfrak{x}}^{\varepsilon}(2 \delta)}(x) .
\end{aligned}
$$

PROOF: To avoid differentiating square roots, define

$$
\begin{array}{rlrl}
\mathfrak{D}^{*}(x) & \stackrel{\text { def }}{=}\left(\tilde{\mathfrak{D}}_{\mathfrak{x}}^{\varepsilon}(x)\right)^{2}=\left(\tilde{\Theta}_{\mathfrak{x}}(x)\right)^{2}+\frac{\tilde{\mathrm{H}}^{4}(x)}{\varepsilon^{4}}, & & x \in \square, \\
\eta^{*}(z) \stackrel{\text { def }}{=} \eta(\sqrt{z}), & z>0 .
\end{array}
$$


Then $\eta^{*}$ is smooth and the support of $\dot{\eta}^{*}$ and $\ddot{\eta}^{*}$ are both contained in the interval $[1,4]$.

Fix $\delta, \varepsilon$, and $x$ as required. Then

$$
\begin{aligned}
\nabla_{E} \mathfrak{D}^{*}(x)= & 2 \tilde{\Theta}_{\mathfrak{x}}(x) \nabla_{E} \tilde{\Theta}_{\mathfrak{x}}(x)+\frac{4}{\varepsilon}\left(\frac{\tilde{\mathrm{H}}(x)}{\varepsilon}\right)^{3} \nabla_{E} \tilde{\mathrm{H}}(x), \\
\left(\tilde{\mathscr{L}}_{\mathfrak{x}} \mathfrak{D}^{*}\right)(x)= & \left\{2 \tilde{\Theta}_{\mathfrak{x}}(x)\left(\tilde{\mathscr{L}}_{\mathfrak{x}} \tilde{\Theta}_{\mathfrak{x}}\right)(x)+\left\|\tilde{\nabla}_{\mathfrak{x}} \tilde{\Theta}_{\mathfrak{x}}(x)\right\|_{E}^{2}\right\} \\
& +\frac{1}{\varepsilon^{2}}\left(\frac{\tilde{\mathrm{H}}(x)}{\varepsilon}\right)^{2}\left\{4 \tilde{\mathrm{H}}(x)\left(\tilde{\mathscr{L}}_{\mathfrak{x}} \tilde{\mathrm{H}}\right)(x)+6\left\|\tilde{\nabla}_{\mathfrak{x}} \tilde{\mathrm{H}}(x)\right\|_{E}^{2}\right\},
\end{aligned}
$$

$\left(\bar{\nabla}_{E} \tilde{\mathrm{H}}, \nabla_{E} \tilde{\mathfrak{D}}^{*}\right)_{E}(x)=2 \tilde{\Theta}_{\mathfrak{x}}(x)\left(\bar{\nabla} \tilde{\nabla}_{E} \tilde{\mathrm{H}}, \nabla_{E} \tilde{\Theta}_{\mathfrak{x}}\right)_{E}(x)$,

and

$$
\begin{aligned}
\left\|\nabla \eta_{\mathfrak{x}}^{\delta, \varepsilon}\left(\tilde{\phi}_{\mathfrak{x}}(x)\right)\right\|= & \left|\dot{\eta}^{*}\left(\frac{\left(\tilde{\mathfrak{D}}_{\mathfrak{x}}^{\varepsilon}(x)\right)^{2}}{\delta^{2}}\right)\right| \frac{\left\|\tilde{\nabla}_{\mathfrak{x}} \mathfrak{D}^{*}(x)\right\|_{E}}{\delta^{2}}, \\
\left(\tilde{\mathscr{L}}_{\mathfrak{x}} \eta_{\mathfrak{x}}^{\delta, \varepsilon}\right)(x)= & \dot{\eta}^{*}\left(\frac{\left(\tilde{\mathfrak{D}}_{\mathfrak{x}}^{\varepsilon}(x)\right)^{2}}{\delta^{2}}\right)\left\{\frac{\left(\bar{\nabla}_{E} \tilde{\mathrm{H}}, \nabla_{E} \tilde{\Theta}_{\mathfrak{x}}\right)_{E}(x)}{\varepsilon^{2} \delta^{2} \tilde{\mathfrak{B}}_{\mathfrak{x}}(x)}+\frac{\left(\tilde{\mathscr{L}}_{\mathfrak{x}} \mathfrak{D}^{*}\right)(x)}{\delta^{2}}\right\} \\
& +\frac{1}{2} \ddot{\eta}^{*}\left(\frac{\left(\tilde{\mathfrak{D}}_{\mathfrak{x}}^{\varepsilon}(x)\right)^{2}}{\delta^{2}}\right) \frac{\left\|\tilde{\nabla}_{\mathfrak{x}} \mathfrak{D}^{*}(x)\right\|_{E}^{2}}{\delta^{4}} .
\end{aligned}
$$

We can then find a positive constant $K$ such that for all $\delta, \varepsilon$, and $x$ as required,

$$
\begin{aligned}
&\left\|\nabla_{E} \mathfrak{D}^{*}(x)\right\| \leq K\left\{\tilde{\mathfrak{D}}_{\mathfrak{x}}^{\varepsilon}(x)+\frac{\left(\tilde{\mathfrak{D}}_{\mathfrak{x}}^{\varepsilon}(x)\right)^{3 / 2}}{\varepsilon}\right\}\left\{\sqrt{\tilde{\mathfrak{D}}_{\mathfrak{x}}^{\varepsilon}(x)}+\varepsilon\right\} \\
&=K \frac{\left(\tilde{\mathfrak{D}}_{\mathfrak{x}}^{\varepsilon}(x)\right)^{2}}{\varepsilon}\left\{1+\frac{\varepsilon}{\sqrt{\tilde{\mathfrak{D}}_{\mathfrak{x}}^{\varepsilon}(x)}}\right\}^{2}, \\
&\left|\left(\tilde{\mathscr{L}}_{\mathfrak{x}} \mathfrak{D}^{*}\right)(x)\right| \leq K\left\{1+\frac{\tilde{\mathfrak{D}}_{\mathfrak{x}}^{\varepsilon}(x)}{\varepsilon^{2}}\right\}\left\{\tilde{\mathfrak{D}}_{\mathfrak{x}}^{\varepsilon}(x)+\varepsilon^{2}\right\} \\
& \mid(\bar{\nabla}\left.\leq K \frac{\left(\tilde{\mathfrak{D}}_{E}^{\varepsilon}(x)\right)^{2}}{\varepsilon^{2}}\left\{1+\frac{\varepsilon^{2}}{\tilde{\mathfrak{D}}_{\mathfrak{x}}^{\varepsilon}(x)}\right\}_{E} \tilde{\mathfrak{D}}^{*}\right)_{E}(x) \mid \\
& \leq K \tilde{\mathfrak{D}}_{\mathfrak{x}}^{\varepsilon}(x)\left\{\tilde{\mathfrak{D}}_{\mathfrak{x}}^{\varepsilon}(x)+\varepsilon^{2}\right\} \\
& \leq K\left(\tilde{\mathfrak{D}}_{\mathfrak{x}}^{\varepsilon}(x)\right)^{2}\left\{1+\frac{\varepsilon^{2}}{\tilde{\mathfrak{D}}_{\mathfrak{x}}^{\varepsilon}(x)}\right\} .
\end{aligned}
$$

The stated results now follow fairly easily, keeping in mind the support of $\dot{\eta}^{*}$ and $\ddot{\eta}^{*}$. 
Proof of Theorem 3.8: First, fix $\delta$ and $\varepsilon$ as required and $\mathfrak{x} \in \mathscr{X}$. If $x \in$ $\mathcal{B}_{\mathfrak{x}}^{\varepsilon}(2 \delta)$, then $|\mathrm{H}(x) / \varepsilon|^{2} \leq 2 \delta$, and hence we have that

$$
\begin{aligned}
\chi_{\mathcal{B}_{\mathfrak{x}}^{\varepsilon}(2 \delta)}(x) & \leq \exp \left[\frac{1}{2} \sqrt{\frac{\pi}{\overline{\mathscr{G}}}}\left\{\sqrt{2}-\left|\frac{\mathrm{H}(x)}{\varepsilon \sqrt{\delta}}\right|\right\}\right] \\
& \leq K_{(3.12)} \exp \left[\sqrt{\frac{\pi}{2 \overline{\mathscr{G}}}}\right] \mathcal{E}\left(\frac{\mathrm{H}(x)}{\varepsilon \sqrt{\delta}}\right), \quad x \in \mathrm{M},
\end{aligned}
$$

and since $\mathcal{E}$ is decreasing on $(0, \infty)$ and is even, we also have that

$$
\chi_{\mathcal{B}_{\mathfrak{x}}^{\varepsilon}(2 \delta)}(x) \leq K_{(3.12)} \exp \left[\sqrt{\frac{\pi}{2 \overline{\mathscr{G}}}}\right] \mathcal{E}\left(\frac{\mathrm{H}(x)}{\varepsilon}\right), \quad x \in \mathrm{M} .
$$

Next, define $\varkappa_{3.8}>0$ to be such that

$$
\varkappa_{3.8}<\min \left\{1, \frac{1}{2^{3 / 2} K_{4.5}}\right\} \text {. }
$$

If $\delta$ and $\varepsilon$ are then as required, we can use Lemma 4.7, and furthermore, Lemma 4.8 tells us that $\|x\|_{\infty}^{2} / \varepsilon^{2} \geq\left(1 / K_{4.8}\right)(\sqrt{\delta} / \varepsilon)$ for all $\mathfrak{x} \in \mathscr{X}$ and $x \in \tilde{\mathcal{B}}_{\mathfrak{x}}^{\varepsilon}(2 \delta) \backslash \tilde{\mathcal{B}}_{\mathfrak{x}}^{\varepsilon}(\delta)$.

We also note that there is a constant $K>0$ such that $\|x\|_{\infty} \leq K\left\|\tilde{\nabla}_{\mathfrak{x}} \tilde{\mathrm{H}}(x)\right\|_{E}^{2}$ for all $x \in \square$ and $\mathfrak{x} \in \mathscr{X}$.

We can now start to bound the errors. We clearly have positive constants $K$ and $K^{\prime}$ such that for all $\delta, \varepsilon$, and $x$ as required

$$
\begin{aligned}
\left|\bar{I}_{1}^{\delta, \varepsilon}(x)\right| & \leq \frac{K}{\sqrt{\delta}} \mathcal{E}\left(\frac{\mathrm{H}(x)}{\varepsilon}\right) \\
\left|\bar{I}_{2}^{\delta, \varepsilon}(x)\right| & \leq K\left\{1+\frac{\|\nabla \mathrm{H}(x)\|^{2}}{\varepsilon}\right\} \sum_{\mathfrak{x} \in \mathscr{X}} \chi_{\mathcal{B}_{\mathfrak{x}}^{\varepsilon}(2 \delta)}(x) \\
& \leq \frac{K^{\prime}}{\sqrt{\delta}} \mathcal{E}\left(\frac{\mathrm{H}(x)}{\varepsilon}\right)+K^{\prime} \frac{\|\nabla \mathrm{H}(x)\|^{2}}{\varepsilon} \mathcal{E}\left(\frac{\mathrm{H}(x)}{\varepsilon}\right) .
\end{aligned}
$$

Fix next $\mathfrak{x} \in \mathscr{X}$. Combining Lemmas 4.4 and 4.7, we can find a constant $K>0$ such that for all $\delta$ and $\varepsilon$ as required and all $x \in \tilde{\mathcal{B}}_{\mathfrak{x}}^{\varepsilon}(2 \delta) \backslash \tilde{\mathcal{B}}_{\mathfrak{x}}^{\varepsilon}(\delta)$,

$$
\begin{gathered}
\left|\tilde{\Phi}_{A, \mathfrak{x}}^{\varepsilon}(x)-\tilde{\Phi}_{B, \mathfrak{x}}^{\varepsilon}(x)\right| \leq K \varepsilon\left\{\varepsilon\|x\|_{\infty}^{2}+\frac{\varepsilon^{2}}{\sqrt{\delta}}\right\}+\frac{K}{\varepsilon} \exp \left[-\frac{1}{K} \frac{\sqrt{\delta}}{\varepsilon}\right] \\
\left\|\nabla_{E}\left(\tilde{\Phi}_{A, \mathfrak{x}}^{\varepsilon}-\tilde{\Phi}_{B, \mathfrak{x}}^{\varepsilon}\right)(x)\right\|_{E} \leq K\left\{\varepsilon+\|x\|_{\infty}^{2}+\frac{\varepsilon}{\sqrt{\delta}}\right\}\|x\|_{\infty}+K \exp \left[-\frac{1}{K} \frac{\sqrt{\delta}}{\varepsilon}\right] .
\end{gathered}
$$

Note that

$$
\left\{\varepsilon+\|x\|_{\infty}^{2}+\frac{\varepsilon}{\sqrt{\delta}}\right\}\|x\|_{\infty} \leq\left\{2 \frac{\varepsilon}{\sqrt{\delta}}+\|x\|_{\infty}^{2}\right\} \varpi
$$


for all $x \in \square$. Combining things and using Lemma 4.9, we can find positive constants $K$ and $K^{\prime}$ such that for all $\delta, \varepsilon$, and $x$ as required

$$
\begin{aligned}
\left|\bar{I}_{i}^{\delta, \varepsilon}(x)\right| & \leq K\left\{\frac{1}{\sqrt{\delta}}+\frac{\|\nabla \mathrm{H}(x)\|^{2}}{\varepsilon}\right\} \chi_{\mathcal{B}_{\mathfrak{x}}^{\varepsilon}(2 \delta)}(x)+\frac{K}{\varepsilon} \exp \left[-\frac{1}{K} \frac{\sqrt{\delta}}{\varepsilon}\right] \\
& \leq \frac{K^{\prime}}{\sqrt{\delta}} \mathcal{E}\left(\frac{\mathrm{H}(x)}{\varepsilon}\right)+K^{\prime} \frac{\|\nabla \mathrm{H}(x)\|^{2}}{\varepsilon} \mathcal{E}\left(\frac{\mathrm{H}(x)}{\varepsilon \sqrt{\delta}}\right)+\frac{K^{\prime}}{\varepsilon} \exp \left[-\frac{1}{K^{\prime}} \frac{\sqrt{\delta}}{\varepsilon}\right]
\end{aligned}
$$

for $i \in\{3,4\}$. Combining things, we get the desired bound on $\mathscr{L}^{\varepsilon} \mathrm{u}^{\delta, \varepsilon}$.

To get the bound on $\mathrm{u}^{\delta, \varepsilon}$ itself, we can use Proposition 3.2 to directly bound the contribution from $\Phi_{A}^{\varepsilon}$, and, by means of (4.3), to bound the contribution of the $\tilde{\Phi}_{B, \mathfrak{x}}^{\varepsilon}$. We get that there are positive constants $K$ and $K^{\prime}$ such that for all $\delta$, $\varepsilon$, and $x$ as required

$$
\left|\mathrm{u}^{\delta, \varepsilon}(x)\right| \leq K \varepsilon \mathcal{E}\left(\frac{\mathrm{H}(x)}{\varepsilon}\right)+K \varepsilon \sum_{\mathfrak{x} \in \mathscr{X}} \chi_{\mathcal{B}_{\mathfrak{x}}^{\varepsilon}(2 \delta)}(x) \leq K^{\prime} \varepsilon .
$$

While Theorem 3.8 gives us bounds on $\mathbf{u}^{\delta, \varepsilon}$ and $\mathscr{L}^{\varepsilon} \mathbf{u}^{\delta, \varepsilon}$, we might also in certain cases want a bound on $\nabla \mathrm{u}^{\delta, \varepsilon}$ (for example, if we multiply $\mathrm{u}^{\delta, \varepsilon}$ by a cutoff function).

LEMMA 4.10 There is a $K>0$ such that for all $\delta \in(0, \bar{\delta})$ and $\varepsilon \in(0,1)$ such that $\varepsilon / \sqrt{\delta} \leq \varkappa_{3.8}$ and for all $x \in \mathrm{M}$ such that $|\mathrm{H}(x)| \geq \varepsilon \sqrt{2 \bar{\delta}}$,

$$
\left\|\nabla \mathrm{u}^{\delta, \varepsilon}(x)\right\| \leq K \mathcal{E}\left(\frac{\mathrm{H}(x)}{\varepsilon}\right) .
$$

Proof: For $\delta, \varepsilon$, and $x$ as required, the fact that $|\mathrm{H}(x)| \geq \varepsilon \sqrt{2 \bar{\delta}}$ implies that $\mathfrak{D}_{\mathfrak{x}}^{\varepsilon}(x) \geq 2 \bar{\delta}$ for all $\mathfrak{x} \in \mathscr{X}$, and hence $\mathrm{u}^{\delta, \varepsilon}(x)=\varepsilon \Phi_{A}^{\varepsilon}(x)$. The claimed estimate follows then from the fact that

$$
\inf \left\{\mathrm{d}_{\ell}\left(\Theta_{\ell}(x)\right)+\left|\frac{\mathrm{H}(x)}{\varepsilon}\right|^{2}: \varepsilon \in(0,1),\left|\frac{\mathrm{H}(x)}{\varepsilon}\right| \geq \sqrt{2 \bar{\delta}}\right\}
$$

is positive for each $\ell \in \Lambda$.

\section{Correctors}

Here we construct some corrector functions to bound the effects of the terms in (3.13) that bound $\mathscr{L}^{\varepsilon} \mathbf{u}^{\delta, \varepsilon}$. See Section 6 of [9] for similar calculations.

Define

$$
\beth(h) \stackrel{\text { def }}{=} 2 \int_{s=0}^{h} \int_{r=0}^{s} \mathcal{E}(r) d r d s, \quad h \in \mathbb{R} .
$$


Then $\frac{1}{2} \ddot{\beth}(h)=\mathcal{E}(h)$ for all $h \in \mathbb{R}$, and there is a constant $K>0$ such that

$$
|\beth(h)| \leq K|h| \quad \text { and } \quad|\dot{\beth}(h)| \leq K, \quad h \in \mathbb{R},
$$

for all $h \in \mathbb{R}$.

For $\delta \in(0, \bar{\delta}), \varepsilon \in(0,1)$, and $x \in \mathrm{M}$, set now

$$
\mathrm{v}_{1}^{\delta, \varepsilon}(x) \stackrel{\text { def }}{=} \varepsilon \delta \beth\left(\frac{\mathrm{H}(x)}{\varepsilon \sqrt{\delta}}\right) .
$$

LEMMA 5.1 There is a constant $K_{5.1}>0$ such that for all $\delta \in(0, \bar{\delta}), \varepsilon \in(0,1)$, and $x \in \mathrm{M}$,

$$
\mathcal{E}\left(\frac{\mathrm{H}(x)}{\varepsilon \sqrt{\delta}}\right) \frac{\|\nabla \mathrm{H}(x)\|^{2}}{\varepsilon} \leq\left(\mathscr{L}^{\varepsilon} \mathrm{v}_{1}^{\delta, \varepsilon}\right)(x)+K_{5.1} \sqrt{\delta} \quad \text { and } \quad\left|\mathrm{v}_{1}^{\delta, \varepsilon}(x)\right| \leq K_{5.1} \sqrt{\delta} .
$$

PRoOF: The bound on $\mathrm{v}_{1}^{\delta, \varepsilon}$ is straightforward from (5.1). For all $\delta, \varepsilon$, and $x$ as required, we also have that

$$
\left(\mathscr{L}^{\varepsilon} \mathrm{v}_{1}^{\delta, \varepsilon}\right)(x)=\mathcal{E}\left(\frac{\mathrm{H}(x)}{\varepsilon \sqrt{\delta}}\right) \frac{\|\nabla \mathrm{H}(x)\|^{2}}{\varepsilon}+\sqrt{\delta} \dot{\beth}\left(\frac{\mathrm{H}(x)}{\varepsilon \sqrt{\delta}}\right)(\mathscr{L} \mathrm{H})(x) .
$$

Again use (5.1).

This takes care of the second term on the right of the second line of (3.13).

To bound the effect of the first term on the right of the second line of (3.13), we need some extra calculations at elements of $\mathscr{X}$, where $\nabla \mathrm{H}$ vanishes. Define

$$
\mathrm{W}(z)=\int_{y=0}^{|z|}\left\{e^{-\frac{y^{2}}{2}} \int_{z=0}^{y} e^{\frac{z^{2}}{2}} d z\right\} d y
$$

for all $z \in \mathbb{R}$. Set

$$
\mathfrak{l}(x) \stackrel{\text { def }}{=} \ln \left(e+|x|^{-1}\right)
$$

for all $x>0$. Then $\mathfrak{l} \geq 1$ and $\mathfrak{l}(x) \approx \ln \frac{1}{|x|}$ when $x \rightarrow 0$.

LEMMA 5.2 We have that

$$
\ddot{\mathrm{W}}(z)+\dot{\mathrm{W}}(z) z=1 \quad \text { and } \quad \dot{\mathrm{W}}(z)=\mathfrak{s}(z) e^{-\frac{z^{2}}{2}} \int_{u=0}^{z} e^{\frac{u^{2}}{2}} d u
$$

for all $z \in \mathbb{R}$. Also, there is a constant $K>0$ such that

$$
|\mathrm{W}(z)| \leq K \mathfrak{l}\left(|z|^{-1}\right) \quad \text { and } \quad|\dot{\mathrm{W}}(z)| \leq \frac{K}{|z|+1}, \quad z \in \mathbb{R} .
$$

PROOF: The expression for $\dot{W}$ is easy to check, and the PDE for $W$ then follows easily. Noting that $W(0)=0$ and that $W$ is even, it suffices to show that there is a constant $K>0$ such that

$$
|\dot{\mathrm{W}}(z)| \leq \frac{K}{|z|+e}
$$


for all $z \in(0, \infty)$. Since both $\dot{W}$ and $x \mapsto 1 /(x+e)$ are continuous on $(0, \infty)$, to prove (5.2), we really only need to check near 0 and near $\infty$. Since $\dot{W}(0)=0$, this bound trivially holds near 0. By l'Hôpital's rule, we can finish the proof upon calculating that

$$
\begin{aligned}
\lim _{x \nearrow \infty}(x+e) \dot{\mathrm{W}}(x) & =\lim _{x \nearrow \infty} \frac{\int_{z=0}^{x} e^{z^{2} / 2} d z}{(x+e)^{-1} e^{x^{2} / 2}} \\
& =\lim _{x \nearrow \infty} \frac{e^{x^{2} / 2}}{\left\{x(x+e)^{-1}-(x+e)^{-2}\right\} e^{x^{2} / 2}}=1 .
\end{aligned}
$$

Set

$$
\mathbf{n}\left(x_{1}, x_{2}\right) \stackrel{\text { def }}{=} x_{1}^{2}+x_{2}^{2}, \quad\left(x_{1}, x_{2}\right) \in \mathbb{R}^{2},
$$

and define the constants

$$
\begin{aligned}
& \alpha \stackrel{\text { def }}{=} \sqrt{\frac{2}{\min \left\{\tilde{\beth}_{\mathfrak{x}}(x) \tilde{g}_{\mathfrak{x}, 1,1}^{-1}(x) \mathrm{B}_{\mathfrak{x}}(x): x \in \square \text { and } \mathfrak{x} \in \mathscr{X}\right\}}}, \\
& \beta \stackrel{2}{=} \frac{2}{\alpha^{2} \min \left\{\tilde{\beth}_{\mathfrak{x}}(x) \tilde{g}_{\mathfrak{x}, 1,1}^{-1}(x): x \in \square \text { and } \mathfrak{x} \in \mathscr{X}\right\}} .
\end{aligned}
$$

Then define

$$
\tilde{\mathbf{w}}^{\varepsilon}(x) \stackrel{\text { def }}{=} \varepsilon^{2} \beta \mathrm{W}\left(\alpha \frac{x_{1}}{\varepsilon}\right) \mathcal{E}\left(\frac{\tilde{\mathrm{H}}(x)}{\varepsilon}\right) \eta\left(\frac{2 \mathbf{n}(x)}{\varpi^{2}}\right)
$$

for all $x=\left(x_{1}, x_{2}\right) \in \square$. Note that $\tilde{\mathbf{w}}^{\varepsilon} \in C_{c}^{\infty}(\square)$.

LEMmA 5.3 There is a constant $K_{5.3}>0$ such that for all $\varepsilon \in(0,1)$ and $x \in \square$,

$$
\begin{aligned}
\mathcal{E}\left(\frac{\tilde{\mathrm{H}}(x)}{\varepsilon}\right) \eta\left(\frac{2 \mathbf{n}(x)}{\varpi^{2}}\right) \leq & \left(\tilde{\mathscr{L}}_{\mathfrak{x}}^{\varepsilon} \tilde{\mathbf{w}}^{\varepsilon}\right)(x)+K_{5.3} \sqrt{\varepsilon} \mathfrak{l}(\varepsilon) \\
& +K_{5.3} \mathfrak{l}(\varepsilon) \mathcal{E}\left(\frac{\tilde{\mathrm{H}}(x)}{\varepsilon}\right) \frac{\left\|\tilde{\nabla}_{\mathfrak{x}} \tilde{\mathrm{H}}(x)\right\|^{2}}{\sqrt{\varepsilon}} \\
\left|\tilde{\mathbf{w}}^{\varepsilon}(x)\right| \leq & K \varepsilon^{2} \mathfrak{l}(\varepsilon) .
\end{aligned}
$$

ProOF: The bound on $\tilde{\mathbf{w}}^{\varepsilon}$ itself is fairly obvious. Define next

$$
\mathbf{x}_{1}\left(x_{1}, x_{2}\right) \stackrel{\text { def }}{=} x_{1} \quad \text { and } \quad \eta^{\dagger}(x) \stackrel{\text { def }}{=} \eta\left(\frac{2 \mathbf{n}(x)}{\varpi^{2}}\right)
$$

for all $x=\left(x_{1}, x_{2}\right) \in \mathbb{R}^{2}$. Then for $\varepsilon$ and $x$ as required,

$$
\left(\tilde{\mathscr{L}}_{\mathfrak{x}}^{\varepsilon} \tilde{\mathbf{w}}^{\varepsilon}\right)(x)=A(x) \mathcal{E}\left(\frac{\tilde{\mathrm{H}}(x)}{\varepsilon}\right) \eta^{\dagger}(x)+\beta \sum_{i=1}^{5} \mathrm{E}_{i}^{\varepsilon}(x)
$$


where

$$
\begin{aligned}
& A(x) \stackrel{\text { def }}{=} \beta\left\{\frac{1}{2} \ddot{\mathrm{W}}\left(\alpha \frac{\mathbf{x}_{1}(x)}{\varepsilon}\right) \alpha^{2} \tilde{\beth}_{\mathfrak{x}}(x) \tilde{g}_{\mathfrak{x}, 1,1}^{-1}(x)+\frac{\alpha \mathbf{x}_{1}(x)}{\tilde{\mathrm{B}}_{\mathfrak{x}}(x) \varepsilon} \dot{\mathrm{W}}\left(\alpha \frac{\mathbf{x}_{1}(x)}{\varepsilon}\right)\right\}, \\
& \mathrm{E}_{1}^{\varepsilon}(x) \stackrel{\text { def }}{=} \frac{1}{\tilde{\mathrm{B}}_{\mathfrak{x}}(x)} \mathrm{W}\left(\alpha \frac{\mathbf{x}_{1}(x)}{\varepsilon}\right) \mathcal{E}\left(\frac{\tilde{\mathrm{H}}(x)}{\varepsilon}\right)\left(\bar{\nabla}_{E} \tilde{\mathrm{H}}, \nabla_{E} \eta^{\dagger}\right)_{E}(x) \\
& +\alpha \dot{\mathbf{W}}\left(\alpha \frac{\mathbf{x}_{1}(x)}{\varepsilon}\right) \dot{\mathcal{E}}\left(\frac{\tilde{\mathrm{H}}(x)}{\varepsilon}\right)\left(\tilde{\nabla}_{\mathfrak{x}} \mathbf{x}_{1}, \tilde{\nabla}_{\mathfrak{x}} \tilde{\mathrm{H}}\right)_{E}(x) \eta^{\dagger}(x), \\
& \mathrm{E}_{2}^{\varepsilon}(x) \stackrel{\text { def }}{=} \frac{1}{2} \mathrm{~W}\left(\alpha \frac{\mathbf{x}_{1}(x)}{\varepsilon}\right) \ddot{\mathcal{E}}\left(\frac{\tilde{\mathrm{H}}(x)}{\varepsilon}\right)\left\|\tilde{\nabla}_{\mathfrak{x}} \tilde{\mathrm{H}}\right\|_{E}^{2}(x) \eta^{\dagger}(x), \\
& \mathrm{E}_{3}^{\varepsilon}(x) \stackrel{\text { def }}{=} \varepsilon \alpha \dot{\mathrm{W}}\left(\alpha \frac{\mathbf{x}_{1}(x)}{\varepsilon}\right) \mathcal{E}\left(\frac{\tilde{\mathrm{H}}(x)}{\varepsilon}\right)\left\{\left(\tilde{\mathscr{L}}_{\mathfrak{x}} \mathbf{x}_{1}\right)(x) \eta^{\dagger}(x)+\left(\tilde{\nabla}_{\mathfrak{x}} \mathbf{x}_{1}, \tilde{\nabla}_{\mathfrak{x}} \eta^{\dagger}\right)_{E}(x)\right\}, \\
& \mathrm{E}_{4}^{\varepsilon}(x) \stackrel{\text { def }}{=} \varepsilon \mathrm{W}\left(\alpha \frac{\mathbf{x}_{1}(x)}{\varepsilon}\right) \dot{\mathcal{E}}\left(\frac{\tilde{\mathrm{H}}(x)}{\varepsilon}\right)\left\{\left(\tilde{\mathscr{L}}_{\mathfrak{x}} \tilde{\mathrm{H}}\right)(x) \eta^{\dagger}(x)+\left(\tilde{\nabla}_{\mathfrak{x}} \tilde{\mathrm{H}}, \tilde{\nabla}_{\mathfrak{x}} \eta^{\dagger}\right)_{E}(x)\right\}, \\
& \mathrm{E}_{5}^{\varepsilon}(x) \stackrel{\text { def }}{=} \varepsilon^{2} \mathrm{~W}\left(\alpha \frac{\mathbf{x}_{1}(x)}{\varepsilon}\right) \mathcal{E}\left(\frac{\tilde{\mathrm{H}}(x)}{\varepsilon}\right)\left(\tilde{\mathscr{L}}_{\mathfrak{x}} \eta^{\dagger}\right)(x) .
\end{aligned}
$$

We first compute that for such $\varepsilon$ and $x$,

$$
\begin{aligned}
A^{\varepsilon}(x)= & \beta\left\{\frac{1}{2} \ddot{\mathrm{W}}\left(\alpha \frac{\mathbf{x}_{1}(x)}{\varepsilon}\right) \alpha^{2} \tilde{\beth}_{\mathfrak{x}}(x) \tilde{g}_{\mathfrak{x}, 1,1}^{-1}(x)+\frac{\alpha \mathbf{x}_{1}(x)}{\tilde{\mathrm{B}}_{\mathfrak{x}}(x) \varepsilon} \dot{\mathrm{W}}\left(\alpha \frac{\mathbf{x}_{1}(x)}{\varepsilon}\right)\right\} \\
= & \beta \frac{\alpha^{2} \tilde{\beth}_{\mathfrak{x}}(x) \tilde{g}_{\mathfrak{x}, 1,1}^{-1}(x)}{2}\left\{\ddot{\mathrm{W}}\left(\alpha \frac{\mathbf{x}_{1}(x)}{\varepsilon}\right)\right. \\
& \left.\quad+\frac{2}{\alpha^{2} \tilde{\beth}_{\mathfrak{x}}(x) \tilde{g}_{\mathfrak{x}, 1,1}^{-1}(x) \tilde{\mathrm{B}}_{\mathfrak{x}}(x)}\left(\frac{\alpha \mathbf{x}_{1}(x)}{\tilde{\mathrm{B}}_{\mathfrak{x}}(x) \varepsilon}\right) \dot{\mathrm{W}}\left(\alpha \frac{\mathbf{x}_{1}(x)}{\varepsilon}\right)\right\} \\
\geq 1 . &
\end{aligned}
$$

Next, we can find a constant $K>0$ such that $\left|\mathrm{W}\left(\alpha \mathbf{x}_{1} / \varepsilon\right)\right| \leq K \mathfrak{l}(\varepsilon)$ for all $\varepsilon$ and $x$ as required. It is thus fairly easy to find a constant $K>0$ such that

$$
\left|\mathrm{E}_{3}^{\varepsilon}(x)\right| \leq K \varepsilon, \quad\left|\mathrm{E}_{4}^{\varepsilon}(x)\right| \leq K \varepsilon \mathfrak{l}(\varepsilon), \quad \text { and } \quad\left|\mathrm{E}_{5}^{\varepsilon}(x)\right| \leq K \varepsilon^{2} \mathfrak{l}(\varepsilon),
$$

for all such $\varepsilon$ and $x$. Note that there is a constant $K>0$ such that $|\dot{\mathcal{E}}(z)| \leq K \mathcal{E}(z)$ and $|\ddot{\mathcal{E}}(z)| \leq K \mathcal{E}(z)$ for all $z \in \mathbb{R}$. Thus there is a constant $K>0$ such that for all 
$\varepsilon$ and $x$ as required,

$$
\begin{aligned}
\left|\mathrm{E}_{2}^{\varepsilon}(x)\right| & \leq K \mathcal{E}\left(\frac{\mathrm{H}(x)}{\varepsilon}\right)\left\|\tilde{\nabla}_{\mathfrak{x}} \tilde{\mathrm{H}}(x)\right\|_{E}^{2}, \\
\left|\mathrm{E}_{1}^{\varepsilon}(x)\right| & \leq K\{\mathfrak{l}(\varepsilon)+1\}\left\|\tilde{\nabla}_{\mathfrak{x}} \tilde{\mathrm{H}}(x)\right\|_{E} \mathcal{E}\left(\frac{\mathrm{H}(x)}{\varepsilon}\right) \\
& \leq 2 K \mathfrak{l}(\varepsilon)\left\|\tilde{\nabla}_{\mathfrak{x}} \tilde{\mathrm{H}}(x)\right\|_{E} \mathcal{E}\left(\frac{\mathrm{H}(x)}{\varepsilon}\right) \\
& \leq K \mathfrak{l}(\varepsilon)\left\{\sqrt{\varepsilon}+\frac{\left\|\tilde{\nabla}_{\mathfrak{x}} \tilde{\mathrm{H}}(x)\right\|_{E}^{2}}{\sqrt{\varepsilon}}\right\} \mathcal{E}\left(\frac{\mathrm{H}(x)}{\varepsilon}\right) \\
& \leq K \mathfrak{l}(\varepsilon) \sqrt{\varepsilon}+K \mathfrak{l}(\varepsilon) \frac{\left\|\tilde{\nabla}_{\mathfrak{x}} \tilde{\mathrm{H}}(x)\right\|_{E}^{2}}{\sqrt{\varepsilon}} \mathcal{E}\left(\frac{\mathrm{H}(x)}{\varepsilon}\right) ;
\end{aligned}
$$

the bound on $E_{2}^{\varepsilon}$ requires Young's inequality.

Let's now construct a second corrector function. Define

$$
\bar{r} \stackrel{\text { def }}{=} \inf \left\{\|\nabla \mathrm{H}(x)\|^{2}: x \notin \bigcup_{\mathfrak{x} \in \mathscr{X}} \tilde{\phi}_{\mathfrak{x}}\left\{x \in \square: \mathbf{n}(x) \leq \varpi^{2}\right\}\right\},
$$

and note that $\bar{r}>0$. For $\varepsilon \in(0,1)$ and $x \in \mathrm{M}$, define

$$
\mathbf{v}_{2}^{\varepsilon}(x) \stackrel{\text { def }}{=} \sum_{\mathfrak{x} \in \mathscr{X}} \tilde{\mathbf{w}}^{\varepsilon}\left(\phi_{\mathfrak{x}}(x)\right) \chi_{\mathbf{B}_{\mathfrak{x}}}(x)+\varepsilon^{2}\left\{\frac{|\mathscr{X}| K_{5.3} \mathfrak{l}(\varepsilon)}{\sqrt{\varepsilon}}+\frac{1}{\bar{r}}\right\} \beth\left(\frac{\mathrm{H}(x)}{\varepsilon}\right) .
$$

LEMMA 5.4 There is a constant $K_{5.4}>0$ such that for all $\varepsilon \in(0,1)$ and $x \in \mathrm{M}$,

$$
\mathcal{E}\left(\frac{\mathrm{H}(x)}{\varepsilon}\right) \leq\left(\mathscr{L}^{\varepsilon} \mathbf{v}_{2}^{\varepsilon}\right)+K_{5.4} \mathfrak{l}(\varepsilon) \sqrt{\varepsilon} \quad \text { and } \quad\left|\mathbf{v}_{2}^{\varepsilon}(x)\right| \leq K_{5.4} \mathfrak{l}(\varepsilon) \sqrt{\varepsilon} .
$$

PROOF: The bound on $\mathbf{v}_{2}^{\varepsilon}$ itself is easy to see. For $\varepsilon$ and $x$ as required, we can compute that

$$
\begin{aligned}
\mathcal{E}\left(\frac{\mathrm{H}(x)}{\varepsilon}\right)= & \sum_{\mathfrak{x} \in \mathscr{X}} \mathcal{E}\left(\frac{\mathrm{H}(x)}{\varepsilon}\right) \eta\left(\frac{2 \mathbf{n}\left(\phi_{\mathfrak{x}}(x)\right)}{\varpi^{2}}\right) \\
& +\mathcal{E}\left(\frac{\mathrm{H}(x)}{\varepsilon}\right)\left\{1-\sum_{\mathfrak{x} \in \mathscr{X}} \eta\left(\frac{2 \mathbf{n}\left(\phi_{\mathfrak{x}}(x)\right)}{\varpi^{2}}\right)\right\} \\
\leq & \sum_{\mathfrak{x} \in \mathscr{X}}\left(\tilde{\mathscr{L}}_{\mathfrak{x}}^{\varepsilon} \tilde{\mathbf{w}}^{\varepsilon}\right)\left(\phi_{\mathfrak{x}}(x)\right)+|\mathscr{X}| K_{5.3} \mathfrak{l}(\varepsilon) \sqrt{\varepsilon} \\
& +\left\{\frac{|\mathscr{X}| K_{5.3} \mathfrak{l}(\varepsilon)}{\sqrt{\varepsilon}}+\frac{1}{\bar{r}}\right\} \mathcal{E}\left(\frac{\mathrm{H}(x)}{\varepsilon}\right)\left\|\nabla_{\mathfrak{x}} \mathrm{H}(x)\right\|^{2} \\
\leq & \left(\mathscr{L}^{\varepsilon} \mathbf{v}_{2}^{\varepsilon}\right)(x)+|\mathscr{X}| K_{5.3} \mathfrak{l}(\varepsilon) \sqrt{\varepsilon} \\
& +\varepsilon\left\{\frac{|\mathscr{X}| K_{5.3} \mathfrak{l}(\varepsilon)}{\sqrt{\varepsilon}}+\frac{1}{\bar{r}}\right\} \dot{\beth}\left(\frac{\mathrm{H}(x)}{\varepsilon}\right)(\mathscr{L} \mathrm{H})(x) .
\end{aligned}
$$


The result is then easy to confirm.

\section{Proof of Main Result}

Proof of Theorem 2.6: For $\varepsilon \in(0,1)$, set

$$
\delta_{\varepsilon} \stackrel{\text { def }}{=} \sqrt{\varepsilon}
$$

this will make several errors of equal size. Then note that for $\varepsilon<\varkappa_{3.8}^{4 / 3}, \varepsilon / \sqrt{\delta_{\varepsilon}}=$ $\varepsilon^{3 / 4}<\varkappa_{3.8}$. For $\varepsilon \in(0,1)$ such that $\varepsilon<\varkappa_{3.8}^{3 / 4}$ and $x \in \mathrm{M}$, define

$$
\begin{gathered}
\Phi^{\varepsilon,+}(x) \stackrel{\text { def }}{=} \mathrm{u}^{\varepsilon, \delta_{\varepsilon}}(x)+K_{3.8} \mathrm{v}_{1}^{\delta_{\varepsilon}, \varepsilon}(x)+\frac{K_{3.8}}{\sqrt{\delta_{\varepsilon}}} \mathrm{v}_{2}^{\varepsilon}(x), \\
\Phi^{\varepsilon,-}(x) \stackrel{\text { def }}{=} \mathrm{u}^{\varepsilon, \delta_{\varepsilon}}(x)-K_{3.8} \mathrm{v}_{1}^{\delta_{\varepsilon}, \varepsilon}(x)-\frac{K_{3.8}}{\sqrt{\delta_{\varepsilon}}} \mathrm{v}_{2}^{\varepsilon}(x) .
\end{gathered}
$$

Then for all such $\varepsilon$ and $x$,

$$
\begin{aligned}
\left|\Phi^{\varepsilon, \pm}(x)\right| & \leq K_{3.8}\left\{\varepsilon+K_{5.1} \sqrt{\delta_{\varepsilon}}+K_{5.4} \mathfrak{l}(\varepsilon) \sqrt{\frac{\varepsilon}{\delta_{\varepsilon}}}\right\} \\
& =K_{3.8}\left\{\varepsilon+K_{5.1} \varepsilon^{\frac{1}{4}}+K_{5.4} \mathfrak{l}(\varepsilon) \varepsilon^{\frac{1}{4}}\right\} .
\end{aligned}
$$

We can then see that for all such $\varepsilon$ and $x$,

$$
\left(\mathscr{L}^{\varepsilon} \Phi^{\varepsilon,+}\right)(x) \geq \mathrm{E}_{\varepsilon} \quad \text { and } \quad\left(\mathscr{L}^{\varepsilon} \Phi^{\varepsilon,-}\right)(x) \leq \mathrm{E}_{\varepsilon}
$$

where

$$
\mathrm{E}_{\varepsilon} \stackrel{\text { def }}{=} K_{3.8}\left\{K_{5.1} \sqrt{\delta_{\varepsilon}}+K_{5.4} \mathfrak{l}(\varepsilon) \sqrt{\frac{\varepsilon}{\delta_{\varepsilon}}}\right\}=K_{3.8}\left\{K_{5.1}+K_{5.4} \mathfrak{l}(\varepsilon)\right\} \varepsilon^{\frac{1}{4}} .
$$

\section{Appendix A: Global Aspects of $\mathfrak{z}$}

For any $x \in \mathrm{M}$ and any subset $S$ of $\mathbb{R}$, define $\mathfrak{z}_{S}(x) \stackrel{\text { def }}{=}\left\{\mathfrak{z}_{t}(x): t \in S\right\}$.

ProOf OF LEMma 2.5: The proof is fairly simple, but we include it for the sake of completeness.

Fix first $x \in \mathrm{M} \backslash \boldsymbol{\Gamma}$. Set $h \stackrel{\text { def }}{=} \mathrm{H}(x)$, and define $L(h) \stackrel{\text { def }}{=}\{x \in \mathrm{M}: \mathrm{H}(x)=h\}$. Note that $\mathfrak{z}_{\mathbb{R}}(x) \subset L(h)$. For $t \in(0, \mathfrak{T}(x))$

$$
t=\int_{z \in \mathfrak{z}[0, t](x)} \frac{1}{\|\bar{\nabla} \mathrm{H}(z)\|} \mathscr{H}^{1}(d z) \leq \int_{x^{\prime} \in L(h)} \frac{1}{\left\|\bar{\nabla} \mathrm{H}\left(x^{\prime}\right)\right\|} \mathscr{H}^{1}\left(d x^{\prime}\right) .
$$

Since $\mathrm{H}$ is continuous, $L(h)$ is a closed subset of the compact set $\overline{\mathrm{M}}$ (recall the choice of $\hbar$ before Definition 2.4) and is thus compact. Since $\bar{\nabla} \mathrm{H}=0$ only on the finite set $\mathscr{X}$, which does not intersect the closed set $L(h), \inf _{x^{\prime} \in L(h)}\left\|\bar{\nabla} \mathrm{H}\left(x^{\prime}\right)\right\|>0$. For each point $x^{\prime} \in L(h)$, since $d H\left(x^{\prime}\right) \neq 0$, there is an open neighborhood $\mathcal{O}$ of $x^{\prime}$ such that $\mathscr{H}^{1}(\mathcal{O} \cap L(h))<1$. Covering $L(h)$ by such open sets and extracting a 
finite subcover, we have that $\mathscr{H}^{1}(L(h))<\infty$. Combining all of these arguments, we have that $\mathfrak{T}(x)<\infty$.

Fix now $x \in \boldsymbol{\Gamma}$. Assume that $\mathfrak{T}(x)<\infty$, and hence that $t \mapsto \mathfrak{z}_{t}(x)$ is periodic. Then $\mathfrak{z}_{\mathbb{R}}(x)=\mathfrak{z}_{[0, \mathfrak{T}(x)]}(x)$, so $\mathfrak{z}_{\mathbb{R}}(x)$ is compact and thus a closed subset of $\boldsymbol{\Gamma}$. On the other hand, periodicity implies that $\bar{\nabla} \mathrm{H} \neq 0$ on all of $\mathfrak{z}_{\mathbb{R}}(x)$. By constructing standard coordinate systems at each point of $\mathfrak{z}_{\mathbb{R}}(x)$ [2, theorem 3.14 , p. 128], we can also easily conclude that $\mathfrak{z}_{\mathbb{R}}(x)$ is an open subset of $\boldsymbol{\Gamma}$. Thus $\mathfrak{z}_{\mathbb{R}}(x)$ is a connected component of $\boldsymbol{\Gamma}$, and by Assumption 2.1, it is in fact all of $\boldsymbol{\Gamma}$. This violates the assumption that $\mathscr{X}$ is nonempty.

Fix now a connected component $\gamma$ of $\Gamma \backslash \mathscr{X}$, and fix $x^{*} \in \gamma$. Standard local analysis [2, theorem 3.14 , p. 128] implies that each $\mathfrak{z}_{\mathbb{R}}\left(x^{*}\right)$ is an open subset of $\boldsymbol{\Gamma} \backslash \mathscr{X}$. We can also see that $\mathfrak{z}_{\mathbb{R}}\left(x^{*}\right)=\overline{\mathfrak{z}_{\mathbb{R}}\left(x^{*}\right)} \cap(\boldsymbol{\Gamma} \backslash \mathscr{X})$, so $\mathfrak{z}_{\mathbb{R}}\left(x^{*}\right)$ is a closed subset of $\boldsymbol{\Gamma} \backslash \mathscr{X}$. We thus have that $\mathfrak{z}_{\mathbb{R}}\left(x^{*}\right)$ is a connected component of $\boldsymbol{\Gamma} \backslash \mathscr{X}$ that intersects $\gamma$; thus, in fact, $\gamma=\mathfrak{z}_{\mathbb{R}}\left(x^{*}\right)$. Similarly, if $\gamma$ is a connected component of $\Gamma_{\ell} \backslash \mathscr{X}$, then $\gamma=\mathfrak{z}_{\mathbb{R}}\left(x^{*}\right)$ for each $x^{*} \in \gamma$.

Proof of Lemma 2.10: Fix $x^{*} \in \Gamma_{\ell} \backslash \mathscr{X}$; we will define $\bar{\Theta}$ on $\mathfrak{z}_{\mathbb{R}}\left(x^{*}\right)$. The map $t \mapsto \mathfrak{z}_{t}\left(x^{*}\right)$ from $\mathbb{R}$ to $\mathfrak{z}_{\mathbb{R}}\left(x^{*}\right)$ is clearly surjective. Since $\Gamma$ contains no periodic orbits, it is also injective; thus the map $t \mapsto \mathfrak{z}_{t}\left(x^{*}\right)$ is a bijection. For each $t \in \mathbb{R}$, we then define

$$
\begin{aligned}
\bar{\Theta}\left(\mathfrak{z}_{t}\left(x^{*}\right)\right) & =\int_{s=0}^{t} \mathfrak{I}\left(\mathfrak{z}_{s}\left(x^{*}\right)\right)\left\|\nabla \mathrm{H}\left(\mathfrak{z}_{s}\left(x^{*}\right)\right)\right\|^{2} d s \\
& =\int_{z \in \in_{[0, s]}\left(x^{*}\right)} \mathrm{J}(z)\|\nabla \mathrm{H}(z)\| \mathscr{H}^{1}(d z) .
\end{aligned}
$$

It is easy to check that $\bar{\Theta}$ is $C^{\infty}$ on $\mathfrak{z}_{\mathbb{R}}\left(x^{*}\right)$. To show that $\bar{\Theta}$ is uniformly continuous, it is sufficient to show that $\bar{\Theta}\left(\mathfrak{z}_{t}\left(x^{*}\right)\right)$ has limits as $t \nearrow \infty$ and $t \searrow-\infty$. This follows from regularity of integration against $\mathscr{H}^{1}$ and from the fact that

$$
\int_{z \in \mathfrak{z} \mathbb{R}\left(x^{*}\right)} I(z)\|\nabla \mathrm{H}(z)\| \mathscr{H}^{1}(d z) \leq \mathscr{G}_{\ell}<\infty .
$$

We now define a useful flow on $M$. This flow will help us define the retract of Lemma 2.15. First, for each $\mathfrak{x} \in \mathscr{X}$, note that

$$
\begin{aligned}
\bar{\nabla}_{E} \tilde{\Theta}_{\mathfrak{x}}\left(x_{1}, x_{2}\right)= & \left.\left\{-2 x_{2} \tilde{\mho}_{\mathfrak{x}}^{V}\left(x_{2}\right)-x_{2}^{2} \frac{\partial \tilde{\mho}_{\mathfrak{x}}^{V}}{\partial x_{2}}\left(x_{2}\right)\right\} \frac{\partial}{\partial x_{1}}\right|_{\left(x_{1}, x_{2}\right)} \\
& +\left.\left\{-2 x_{1} \tilde{\mho}_{\mathfrak{x}}^{H}\left(x_{1}\right)-x_{1}^{2} \frac{\partial \tilde{\mho}_{\mathfrak{x}}^{H}}{\partial x_{1}}\left(x_{1}\right)\right\} \frac{\partial}{\partial x_{2}}\right|_{\left(x_{1}, x_{2}\right)}
\end{aligned}
$$


for all $\left(x_{1}, x_{2}\right) \in \check{\square}$. Define the vector field

$$
\mathbf{V}_{\mathfrak{x}}^{\circ}(x) \stackrel{\text { def }}{=} D \tilde{\phi}_{\mathfrak{x}} \bar{\nabla}_{E} \tilde{\Theta}_{\mathfrak{x}}\left(\phi_{\mathfrak{x}}(x)\right)
$$

for all $x=\left(x_{1}, x_{2}\right) \in \check{\square}$. Note that

$$
\begin{aligned}
\left(\mathbf{V}_{\mathfrak{x}}^{\circ}, \nabla \mathrm{H}\right) & \left(\tilde{\phi}_{\mathfrak{x}}(x)\right)=\left(\bar{\nabla}_{E} \tilde{\Theta}_{\mathfrak{x}}\left(\mathrm{H} \circ \tilde{\phi}_{\mathfrak{x}}\right)\right)(x)=\left(\bar{\nabla}_{E} \tilde{\Theta}_{\mathfrak{x}}, \nabla_{E} \tilde{\mathrm{H}}\right)_{E}(x)= \\
& -2\left\{x_{1}^{2} \tilde{\mho}_{\mathfrak{x}}^{H}\left(x_{1}\right)+x_{2}^{2} \tilde{\mho}_{\mathfrak{x}}^{V}\left(x_{2}\right)\right\}-\left\{x_{1}^{3} \frac{\partial \tilde{\mho}_{\mathfrak{x}}^{H}}{\partial x_{1}}\left(x_{1}\right)+x_{2}^{3} \frac{\partial \tilde{\mho}_{\mathfrak{x}}^{V}}{\partial x_{2}}\left(x_{2}\right)\right\}
\end{aligned}
$$

for all $x \in \check{\square}$. Since $\tilde{\mho}_{\mathfrak{x}}^{H}(0)$ and $\tilde{\mho}_{\mathfrak{x}}^{V}(0)$ are both positive and $C^{\infty}$ on $\check{\square}$, there is an open neighbhorhood $\mathcal{O}_{1}$ of $\mathbf{0}$ that is contained in $\square$ and such that $\left(\mathbf{V}_{\mathfrak{x}}^{\circ}, \nabla \mathrm{H}\right)<0$ on $\tilde{\phi}_{\mathfrak{x}}\left(\mathcal{O}_{1}\right) \backslash\{\mathfrak{x}\}$. Let $\mathcal{O}_{2}$ be any neighborhood of $\mathbf{0}$ such that $\overline{\mathcal{O}_{2}} \subset \mathcal{O}_{1}$; then by standard extension methods, there are $\alpha_{1}$ and $\alpha_{2}$ in $C^{\infty}(\mathrm{M} \backslash \mathscr{X})$ such that

$$
\alpha_{1}=\frac{\left(\mathbf{V}_{\mathfrak{x}}^{\circ}, \nabla \mathrm{H}\right)}{\|\nabla \mathrm{H}\|} \quad \text { and } \quad \alpha_{2}=\frac{\left(\mathbf{V}_{\mathfrak{x}}^{\circ}, \bar{\nabla} \mathrm{H}\right)}{\|\nabla \mathrm{H}\|}
$$

on $\mathcal{O}_{2}$ and such that $\alpha_{1}<0$ on $\mathrm{M} \backslash \mathscr{X}$. Define then

$$
\mathbf{V} \stackrel{\text { def }}{=} \chi_{\mathrm{M} \backslash \mathscr{X}}\left\{\alpha_{1} \frac{\nabla \mathrm{H}}{\|\nabla \mathrm{H}\|}+\alpha_{2} \frac{\bar{\nabla} \mathrm{H}}{\|\nabla \mathrm{H}\|}\right\}
$$

on all of $\mathbf{M}$; then $\mathbf{V}$ is a smooth vector field on $\mathrm{M}$ such that $\mathbf{V}=\mathbf{V}_{\mathfrak{x}}^{\circ}$ on $\tilde{\phi}_{\mathfrak{x}}\left(\mathcal{O}_{2}\right)$ and such that $(\mathbf{V}, \nabla \mathrm{H})<0$ on $\mathrm{M} \backslash \mathscr{X}$. Note that this last fact implies that $\{x \in \mathrm{M} \backslash \mathscr{X}$ : $\mathbf{V}(x)=0\}=\mathscr{X}$. Define next the vector field

$$
\check{\mathbf{V}} \stackrel{\text { def }}{=} \frac{1}{(\mathbf{V}, \nabla H)} \nabla \mathrm{H}
$$

on $\mathrm{M} \backslash \mathscr{X}$.

Fix now $x \in \mathrm{M}$. If $x \in \mathrm{M} \backslash \mathscr{X}$, we let $\left\{\wp_{t}^{*}(x): t \in I_{x}\right\}$ be the maximal solution $[2$, pp. 126, 134] of the ODE

$$
\begin{aligned}
& \dot{\wp}_{t}^{*}(x)=-\mathrm{H}(x) \check{\mathbf{V}}\left(\wp_{t}^{*}(x)\right), \quad t \in I_{x}, \\
& \wp_{0}^{*}(x)=x .
\end{aligned}
$$

If $x \in \mathscr{X}$, we define $I_{x} \stackrel{\text { def }}{=} \mathbb{R}$ and set $\wp_{t}^{*}(x) \stackrel{\text { def }}{=} x$ for all $t \in I_{x}$. If $x \in \boldsymbol{\Gamma}$, then by uniqueness $I_{x}=\mathbb{R}$ and $\wp_{t}^{*}(x)=x$ for all $t \in \mathbb{R}$. The ODE (A.3) implies that for $x \in \mathrm{M}$,

$$
\mathrm{H}\left(\wp_{t}^{*}(x)\right)=\mathrm{H}(x)(1-t), \quad t \in I_{x} .
$$

If $x \in \mathrm{M} \backslash \boldsymbol{\Gamma}, \wp_{t}^{*}(x) \notin \mathscr{X}$ for all $t \in[0,1) \cap I_{x}$, so by uniqueness of maximal solutions, $I_{x} \supset[0,1)$. In sum, $I_{x} \supset[0,1)$ for all $x \in \mathrm{M}$. 
Similarly, for any $x \in \mathrm{M}$, we let $\left\{\mathfrak{y}_{t}(x): t \in I_{x}^{\prime}\right\}$ be the maximal solution of the ODE

$$
\begin{aligned}
\dot{\mathfrak{y}}_{t}(x) & =\mathbf{V}\left(\mathfrak{y}_{t}(x)\right), \quad t \in I_{x}^{\prime}, \\
\mathfrak{y}_{0}(x) & =x .
\end{aligned}
$$

Remark A.1. It is easy to see from (A.1) that each $\mathfrak{x}$ is a hyperbolic fixed point of the vector field V. Defining

$$
\begin{aligned}
& \mathscr{W}_{\mathfrak{x}}^{s} \stackrel{\text { def }}{=}\left\{x \in \mathrm{M}: \mathbb{R}_{+} \subset I_{x}^{\prime} \text { and } \lim _{t \nearrow \infty} \mathfrak{y}_{t}(x)=\mathfrak{x}\right\}, \\
& \mathscr{W}_{\mathfrak{x}}^{u} \stackrel{\text { def }}{=}\left\{x \in \mathrm{M}: \mathbb{R}_{-} \subset I_{x}^{\prime} \text { and } \lim _{t \searrow-\infty} \mathfrak{y}_{t}(x)=\mathfrak{x}\right\},
\end{aligned}
$$

we have that both $\mathscr{W}_{\mathfrak{x}}^{s}$ and $\mathscr{W}_{\mathfrak{x}}^{u}$ are immersed one-dimensional submanifolds of $\mathrm{M}$. If $x \notin \mathrm{X}_{\mathfrak{r}} \stackrel{\text { def }}{=} \mathscr{W}_{\mathfrak{x}}^{u} \cup \mathscr{W}_{\mathfrak{x}}^{s}$, then $\overline{\left\{\wp_{t}^{*}(x): t \in I_{x}\right\}}$ is contained in $\overline{\left\{\mathfrak{y}_{t}(x): t \in I_{x}^{\prime}\right\}}$, which does not intersect $\mathfrak{x}$.

We next claim that for all $x \in M$, the limit

$$
\wp(x) \stackrel{\text { def }}{=} \lim _{\substack{t \nearrow 1 \\ t \in[0,1)}} \wp_{t}^{*}(x)
$$

is well-defined. If $x \in \mathrm{M} \backslash \bigcup_{\mathfrak{x} \in \mathscr{X}} \mathrm{X}_{\mathfrak{x}}$, then the vector field $\left\{\mathrm{H}(x) \check{\mathbf{V}}\left(\wp_{t}^{*}(x)\right)\right.$ : $t \in[0,1)\}$ is bounded by Remark A.1 ( $(\check{\mathbf{V}}$ only blows up near $\mathscr{X}$ ), so the map $t \mapsto \wp_{t}^{*}(x)$ from $[0,1)$ to $\mathrm{M}$ is uniformly continuous. Assume next that $x \in \mathrm{X}_{\mathfrak{x}}$. Since $\bar{M}$ is compact, we may and will assume that $\lim _{n \rightarrow \infty} \wp_{t_{n}}^{*}(x)$ exists for some sequence $\left(t_{n}: n \in N\right)$ such that $t_{n} \nearrow 1$. The limit point must then be in $\{x \in$ $\left.\overline{\mathrm{X}_{\mathfrak{x}}}: \mathrm{H}(x)=0\right\}=\{\mathfrak{x}\}$; thus $\lim _{t \nearrow 1} \wp_{t}^{*}(x)=\mathfrak{x}$. We claim that (A.5) is the desired retract. Note that we have proven that $X_{\mathfrak{x}} \subset \wp^{-1}(\{\mathfrak{x}\})$. Remark A.1 implies that $\wp(x) \neq \mathfrak{x}$ if $x \in \mathrm{M} \backslash \mathrm{X}_{\mathfrak{x}}$, so $\mathrm{X}_{\mathfrak{x}}=\wp^{-1}(\{\mathfrak{x}\})$.

Proof OF Lemma 2.15: We can easily see (using (A.4) if $x \in M \backslash \Gamma$ ) that $\wp(x) \in \boldsymbol{\Gamma}$ for all $x \in \mathrm{M}$; thus $\wp: \mathrm{M} \rightarrow \boldsymbol{\Gamma}$. We can also easily see that $\wp$ is the identity map on $\boldsymbol{\Gamma}$. Furthermore, $\mathbf{X} \stackrel{\text { def }}{=} \wp^{-1}(\mathscr{X})=\bigcup_{\mathfrak{x} \in \mathscr{X}} \mathbf{X}_{\mathfrak{x}}$, and this is indeed a one-dimensional immersed submanifold of $\mathrm{M}$.

Fix now $x \in M \backslash \mathbf{X}$. Using Remark A.1 and then smoothing the vector field near $\mathscr{X}$, standard results on the regularity of solutions of ODEs with respect to initial conditions and parameters imply that $\wp$ is $C^{\infty}$ at $x$.

Let's next fix $\mathfrak{x} \in \mathscr{X}$ and look at $\wp$ in a neighborhood of $\mathfrak{x}$. First of all, define the vector field

$$
\tilde{\mathbf{W}}_{\mathfrak{x}} \stackrel{\text { def }}{=} \frac{\bar{\nabla}_{E} \tilde{\Theta}_{\mathfrak{x}}}{\left(\bar{\nabla}_{E} \tilde{\Theta}_{\mathfrak{x}}, \nabla_{E} \tilde{\mathrm{H}}\right)_{E}}
$$

for $x \in \mathcal{O}_{2} \backslash\{0\}$. Then $\check{\mathbf{V}}(x)=D \tilde{\phi}_{\mathfrak{x}} \tilde{\mathbf{W}}_{\mathfrak{x}}\left(\phi_{\mathfrak{x}}(x)\right)$ for all $x \in \tilde{\phi}_{\mathfrak{x}}\left(\mathcal{O}_{2}\right) \backslash\{\mathfrak{x}\}$. Recall $\mathbf{n}$ of (5.3). Using (A.2), it is fairly easy to find a $K>0$ such that $\left|\left(\tilde{\mathbf{W}}_{\mathfrak{x}}, \nabla_{E} \mathbf{n}\right)_{E}\right| \leq K$ 
for all $x \in \mathcal{O}_{2} \backslash\{\boldsymbol{0}\}$ and all $\mathfrak{x} \in \mathscr{X}$. Thus if $x \in \mathcal{O}_{2}$ and $\phi_{\mathfrak{x}}\left(\wp_{t}^{*}\left(\tilde{\phi}_{\mathfrak{x}}(x)\right)\right) \in \mathcal{O}_{2}$ for all $0<t<t^{*}$ for some $t^{*} \in(0,1)$, then

$$
\begin{aligned}
\left\|\phi_{\mathfrak{x}}\left(\wp_{t}^{*}\left(\tilde{\phi}_{\mathfrak{x}}(x)\right)\right)\right\|_{\infty}^{2} & \leq \mathbf{n}\left(\phi_{\mathfrak{x}}\left(\wp_{t}^{*}\left(\tilde{\phi}_{\mathfrak{x}}(x)\right)\right)\right) \\
& \leq \mathbf{n}(x)+\left|\mathrm{H}\left(\tilde{\phi}_{\mathfrak{x}}(x)\right)\right| K t \\
& \leq \mathbf{n}(x)+|\tilde{\mathrm{H}}(x)| K t \\
& \leq \mathbf{n}(x)\left\{1+\frac{K t}{2}\right\} \\
& \leq\|x\|_{\infty}^{2}\{2+K\} .
\end{aligned}
$$

Fix now $\varpi^{*} \in(0,1)$ such that $\left\{x \in \mathbb{R}^{2}:\|x\|_{\infty} \leq \varpi^{*}\right\} \subset \mathcal{O}_{2}$. Define then

$$
\mathcal{O}_{3} \stackrel{\text { def }}{=}\left\{x \in \mathcal{O}_{2}:\|x\|_{\infty}<\frac{\varpi^{*}}{\sqrt{2+K}}\right\} \text {. }
$$

If $x \in \mathcal{O}_{3}$ and $y \in \mathbb{R}^{2}$ is such that $\|y\|_{\infty}^{2} \leq(2+K)\|x\|_{\infty}^{2}$, then $\|y\|_{\infty} \leq \varpi^{*}$ and hence $y \in \mathcal{O}_{2}$. The above calculation means that if $x \in \mathcal{O}_{3}$, then

$$
\overline{\left\{\phi_{\mathfrak{x}}\left(\wp_{t}^{*}\left(\tilde{\phi}_{\mathfrak{x}}(x)\right)\right): t \in[0,1)\right\}}
$$

is contained in $\mathcal{O}_{2}$. Thus if $x \in \mathcal{O}_{3}$, then $\phi_{\mathfrak{x}}\left(\wp\left(\tilde{\phi}_{\mathfrak{x}}(x)\right)\right) \in \mathcal{O}_{2}$ and

$$
\tilde{\Theta}_{\mathfrak{x}}\left(\phi_{\mathfrak{x}}\left(\wp\left(\tilde{\phi}_{\mathfrak{x}}(x)\right)\right)\right)=\lim _{t \nearrow 1} \tilde{\Theta}_{\mathfrak{x}}\left(\phi_{\mathfrak{x}}\left(\wp_{t}^{*}\left(\tilde{\phi}_{\mathfrak{x}}(x)\right)\right)\right)=\tilde{\Theta}_{\mathfrak{x}}(x) .
$$

Set $\mathscr{V}_{\mathfrak{x}} \stackrel{\text { def }}{=} \tilde{\phi}_{\mathfrak{x}}\left(\mathcal{O}_{3}\right)$. Note that $\overline{\wp\left(\mathscr{V}_{\mathfrak{x}}\right)} \subset \overline{\tilde{\phi}_{\mathfrak{x}}\left(\mathcal{O}_{2}\right)} \subset \tilde{\phi}_{\mathfrak{x}}\left(\mathcal{O}_{1}\right) \subset \tilde{\phi}_{\mathfrak{x}}(\check{\square})$.

We now return to the issue of continuity. We already know that $\wp$ is $C^{\infty}$ on $\mathrm{M} \backslash \mathbf{X}$; fixing a $\mathfrak{x} \in \mathscr{X}$, we need to show that $\wp$ is continuous at $\mathrm{X}_{\mathfrak{x}}$. Fix $x \in \mathscr{V}_{\mathfrak{x}} \cap \mathbf{X}_{\mathfrak{x}}$. Assume that $\left(x_{n}: n \in \mathbb{N}\right)$ is a sequence of points in $\mathrm{M}$ such that $\lim _{n \rightarrow \infty} x_{n}=x$. Since $\bar{M}$ is compact, we may and will assume that $z \stackrel{\text { def }}{=} \lim _{n \rightarrow \infty} \wp\left(x_{n}\right)$ exists. Since $\mathscr{V}_{\mathfrak{x}}$ is open, $x_{n} \in \mathscr{V}_{\mathfrak{x}}$ for sufficiently large $n$, so $z \in \overline{\wp\left(\mathscr{V}_{\mathfrak{x}}\right)} \subset \tilde{\phi}_{\mathfrak{x}}(\check{\square})$. By continuity of $\tilde{\Theta}_{\mathfrak{x}}$ on $\breve{\square}$, we thus have that

$$
\begin{aligned}
\tilde{\Theta}_{\mathfrak{x}}\left(\phi_{\mathfrak{x}}(z)\right)=\lim _{n \rightarrow \infty} \tilde{\Theta}_{\mathfrak{x}}\left(\phi_{\mathfrak{x}}\left(\wp\left(x_{n}\right)\right)\right)= & \lim _{n \rightarrow \infty} \tilde{\Theta}_{\mathfrak{x}}\left(\phi_{\mathfrak{x}}\left(x_{n}\right)\right)=\tilde{\Theta}_{\mathfrak{x}}\left(\phi_{\mathfrak{x}}(x)\right)= \\
& \tilde{\Theta}_{\mathfrak{x}}\left(\phi_{\mathfrak{x}}(\wp(x))\right)=\tilde{\Theta}_{\mathfrak{x}}\left(\phi_{\mathfrak{x}}(\mathfrak{x})\right)=\tilde{\Theta}_{\mathfrak{x}}(\mathbf{0})=0
\end{aligned}
$$

and thus $\phi_{\mathfrak{x}}(z) \in\left\{x \in \tilde{\boldsymbol{\Gamma}}: \tilde{\Theta}_{\mathfrak{x}}(x)=0\right\}=\{\mathbf{0}\}$ so $z=\mathfrak{x}$. In other words, $\lim _{n \rightarrow \infty} \wp\left(x_{n}\right)=\mathfrak{x}=\wp(x)$, so $\wp$ is continuous on $\mathscr{V}_{\mathfrak{x}} \cap \mathbf{X}_{\mathfrak{x}}$, and more generally on all of $\mathscr{V}_{\mathfrak{x}}$ by using the previously verified continuity. Fix next any $x \in \mathrm{X}_{\mathfrak{x}}$. Then there is a $t \in(0,1)$ such that $\wp_{t}^{*}(x) \in \mathscr{V}_{\mathfrak{x}}$. Furthermore, there is then a small neighborhood $\mathcal{O}$ of $x$ that is contained in $\mathrm{M}$ such that $\wp_{t}^{*}\left(x^{\prime}\right) \in \mathscr{V}_{\mathfrak{x}}$ for all $x^{\prime} \in \mathcal{O}$. For $x^{\prime} \in \mathcal{O}$, we have that $\wp\left(x^{\prime}\right)=\wp\left(\wp_{t}^{*}\left(x^{\prime}\right)\right)$ by uniqueness of solutions of ODEs. The continuity of $\wp_{t}^{*}$ on $\mathcal{O}$ and the continuity of $\wp$ on $\mathscr{V}_{\mathfrak{x}}$ give us continuity at $x$. 
Fixing a $\mathfrak{x} \in \mathscr{X}$, we finally need to show that $\bar{\Theta}_{\mathfrak{x}}^{\sim} \circ \phi_{\mathfrak{x}} \circ \wp$ is $C^{\infty}$ on $\wp^{-1}\left(\tilde{\phi}_{\mathfrak{x}}(\check{\square})\right)$. By previous smoothness arguments, we really only need to show that $\bar{\Theta}_{\mathfrak{x}}^{\sim} \circ \phi_{\mathfrak{x}} \circ \wp$ is $C^{\infty}$ at all points of $X_{\mathfrak{x}}$. Since $\bar{\Theta}_{\mathfrak{x}}^{\sim} \circ \phi_{\mathfrak{x}} \circ \wp=\tilde{\Theta}_{\mathfrak{x}} \circ \phi_{\mathfrak{x}}$ on $\mathscr{V}_{\mathfrak{x}}, \bar{\Theta}_{\mathfrak{x}}^{\sim} \circ \phi_{\mathfrak{x}} \circ \wp$ is clearly $C^{\infty}$ on $\mathscr{V}_{\mathfrak{x}}$. Fix next $x \in \mathrm{X}_{\mathfrak{x}}$. As above, there is a $t \in(0,1)$ such that $\wp_{t}^{*}(x) \in \mathscr{V}_{\mathfrak{x}}$ and a small neighborhood $\mathcal{O}$ of $x$ that is contained in $\mathrm{M}$ such that $\wp_{t}^{*}\left(x^{\prime}\right) \in \mathscr{V}_{\mathfrak{x}}$ for all $x^{\prime} \in \mathcal{O}$. For $\left.x^{\prime} \in \mathcal{O}, \bar{\Theta}_{\mathfrak{x}}^{\sim} \circ \phi_{\mathfrak{x}} \circ \wp\left(x^{\prime}\right)=\left(\bar{\Theta}_{\mathfrak{x}}^{\sim} \circ \phi_{\mathfrak{x}} \circ \wp\right)(\wp)_{t}^{*}\left(x^{\prime}\right)\right)$, so the smoothness of $\bar{\Theta}_{\mathfrak{x}}^{\sim} \circ \phi_{\mathfrak{x}} \circ \wp$ at $x$ follows from the smoothness of $\bar{\Theta}_{\mathfrak{x}}^{\sim} \circ \phi_{\mathfrak{x}} \circ \wp$ on $\mathscr{V}_{\mathfrak{x}}$ and the smoothness of $\wp_{t}^{*}$ on $\mathcal{O}$.

Let's next characterize $\mathrm{M}_{\ell}$.

Lemma A.2 Fix $x^{*} \in \mathrm{M}_{\ell}$. Then

$$
\mathrm{M}_{\ell}=\left\{\wp_{\lambda}^{*}\left(\mathfrak{z}_{t}\left(x^{*}\right)\right): t \in \mathbb{R} \text { and }-\frac{ \pm \hbar-\mathrm{H}\left(x^{*}\right)}{\mathrm{H}\left(x^{*}\right)}<\lambda<1\right\},
$$

where we choose + if $\ell \in \Lambda_{P}$ and - if $\ell \in \Lambda_{W}$.

PROOF: For convenience define

$$
J \stackrel{\text { def }}{=}\left(-\frac{ \pm \hbar-\mathrm{H}\left(x^{*}\right)}{\mathrm{H}\left(x^{*}\right)}, 1\right) \text { and } \quad A \stackrel{\text { def }}{=}\left\{\wp_{\lambda}^{*}\left(\mathfrak{z}_{t}\left(x^{*}\right)\right):(t, \lambda) \in \mathbb{R} \times J\right\} .
$$

It is fairly easy to see that the map $(t, \lambda) \mapsto \wp_{\lambda}^{*}\left(\mathfrak{z}_{t}\left(x^{*}\right)\right)$ from $\mathbb{R} \times J$ to $\mathrm{M}$ is full rank, so it is a local diffeomorphism. Hence $A$ is an open subset of $\mathbf{M}_{\ell}$. Assume next that $z_{n}=\wp_{\lambda_{n}}^{*}\left(\mathfrak{z}_{t_{n}}\left(x^{*}\right)\right)$ for each $n$ and that $z \stackrel{\text { def }}{=} \lim _{n} z_{n}$ exists and is in $\mathrm{M}_{\ell}$. Then

$$
\lambda_{\infty} \stackrel{\text { def }}{=} 1-\frac{\mathrm{H}(z)}{\mathrm{H}\left(x^{*}\right)}=\lim _{n \rightarrow \infty} 1-\frac{\mathrm{H}\left(z_{n}\right)}{\mathrm{H}\left(x^{*}\right)}=\lim _{n \rightarrow \infty} \lambda_{n} .
$$

For $n \in \mathbb{N}$, define next

$$
\hat{t}_{n} \stackrel{\text { def }}{=} t_{n}-\left\lfloor\frac{t_{n}}{\mathfrak{T}\left(x^{*}\right)}\right\rfloor \mathfrak{T}\left(x^{*}\right) .
$$

Since $\mathfrak{T}\left(x^{*}\right)<\infty$, we can assume (by extracting a subsequence if necessary) that $\hat{t}_{\infty} \stackrel{\text { def }}{=} \lim _{n \rightarrow \infty} \hat{t}_{n}$ exists. Then

$$
z=\lim _{n \rightarrow \infty} \wp_{\lambda_{n}}^{*}\left(\mathfrak{z}_{t_{n}}\left(x^{*}\right)\right)=\wp_{\lambda_{\infty}}^{*}\left(\mathfrak{z}_{\hat{t}_{\infty}}\left(x^{*}\right)\right)
$$

so $z \in A$. Hence $A$ is a closed subset of $\mathrm{M}_{\ell}$. Being both closed and open in the topology inherited from $\mathrm{M}_{\ell}, A=\mathrm{M}_{\ell}$.

Proof of Lemma 2.11: Fix $x^{*} \in \mathbf{X} \cap \mathrm{M}_{\ell}$ and define

$$
\begin{aligned}
q(t) & \stackrel{\text { def }}{=} \wp\left(\mathfrak{z}_{t}\left(x^{*}\right)\right), \quad t \in \mathbb{R}, \\
H_{1}(\lambda, t) & \stackrel{\text { def }}{=} \wp_{\lambda}^{*}\left(\mathfrak{z}_{t}\left(x^{*}\right)\right), \quad \lambda \in[0,1], t \in \mathbb{R} .
\end{aligned}
$$

Clearly $q$ is continuous and the maps $t \mapsto \mathfrak{z}_{t}\left(x^{*}\right)$ and $t \mapsto q(t)$ from $\mathbb{R}$ to $\mathrm{M}_{\ell} \cup \Gamma_{\ell}$ are homotopic; also $q(0) \in \mathscr{X}$. Defining $\hat{A} \stackrel{\text { def }}{=}\{t \in \mathbb{R}: q(t) \notin \mathscr{X}\}$, we have that $q$ 
is $C^{\infty}$ on $\hat{A}$; since $\mathbb{R} \backslash \hat{A}=\{t \in \mathbb{R}: q(t) \in \mathscr{X}\} \subset\left\{t \in \mathbb{R}: \mathfrak{z}_{t}\left(x^{*}\right) \in \mathbf{X}\right\}$ and $\bar{\nabla} \mathrm{H}$ is transversal to $\mathbf{X} \backslash \mathscr{X}, \mathbb{R} \backslash \hat{A}$ is discrete.

We first claim that $(\dot{q}, \bar{\nabla} \mathrm{H}(q))>0$ on $\hat{A}$. Set $h \stackrel{\text { def }}{=} \mathrm{H}\left(x^{*}\right)$, fix $t \in \hat{A}$, and define $\bar{x} \stackrel{\text { def }}{=} \mathfrak{z}_{t}\left(x^{*}\right)$. By Remark A.1, we can solve the ODE

$$
\begin{aligned}
& \dot{r}_{\lambda}(x)=h \mathbf{V}\left(r_{\lambda}(x)\right), \quad \lambda \in[0,1], \\
& r_{0}(x)=x,
\end{aligned}
$$

for $x \in \mathfrak{z}_{(t-\delta, t+\delta)}\left(x^{*}\right)$ for some sufficiently small $\delta$. By uniqueness of solutions of ODEs, we must have that $r_{\lambda}(x)=\wp_{\lambda}^{*}(x)$ for all $x \in \mathfrak{z}_{(t-\delta, t+\delta)}\left(x^{*}\right)$. Then $\dot{q}(t)=$ $D r_{1}(\bar{\nabla} \mathrm{H}(\bar{x}))$. The structure of (A.6) implies that there is an $a \in C([0,1])$ such that $D r_{\lambda}(\bar{\nabla} \mathrm{H}(\bar{x}))=a_{\lambda} \bar{\nabla} \mathrm{H}\left(r_{\lambda}(\bar{x})\right)$ for all $\lambda \in[0,1]$. We have that $a_{0}=1$, and since $D r_{\lambda}$ is full rank on $T_{\bar{x}} \mathrm{M}$ for all $\lambda \in[0,1]$, we must have that $a_{\lambda} \neq 0$ for all $\lambda \in[0,1]$. By connectedness, $(\dot{q}(t), \bar{\nabla} \mathrm{H}(q(t)))>0$.

Define now

$$
\tau(t) \stackrel{\text { def }}{=} \int_{s=0}^{t} \mathrm{~J}(q(s))(\dot{q}(s), \bar{\nabla} \mathrm{H}(q(s))) d s, \quad t \in \mathbb{R} .
$$

Clearly $\tau$ is continuous and strictly increasing and $C^{\infty}$ on $\hat{A}$. Furthermore, $\dot{\tau}>0$ on $\hat{A}$. Thus its inverse $\tau^{*} \stackrel{\text { def }}{=} \tau^{-1}$ is also continuous and strictly increasing, and $C^{\infty}$ on $\tau(\hat{A})$. Furthermore, $\tau(\hat{A})$ is discrete, and $\dot{\tau}^{*}(t)>0$ on $\tau(\hat{A})$. We then define

$$
\mathbf{p}_{\ell}(t) \stackrel{\text { def }}{=} q\left(\tau^{*}(t)\right), \quad t \in \mathbb{R} .
$$

Clearly $\mathbf{p}_{\ell}$ is continuous and $C^{\infty}$ on $\tau(\hat{A})=A_{\ell}$ (where $A_{\ell}$ was given in the statement of the result). Defining

$$
H_{2}(\lambda, t) \stackrel{\text { def }}{=} q\left(\lambda \tau^{*}(t)+(1-\lambda) t\right)
$$

for all $\lambda \in[0,1)$ and $t \in \mathbb{R}$, we then see that the maps $q$ and $\mathbf{p}_{\ell}$ from $\mathbb{R}$ to $\mathrm{M}_{\ell} \cup \Gamma_{\ell}$ are homotopic.

Next, for any $x \in \mathrm{M}_{\ell}$, we claim that the maps $t \mapsto \mathfrak{z}_{t}(x)$ and $t \mapsto \mathfrak{z}_{t}\left(x^{*}\right)$ from $\mathbb{R}$ to $\mathrm{M}_{\ell} \cup \Gamma_{\ell}$ are homotopic. Since $\mathrm{M}_{\ell}$ is connected, it is arcwise connected, so there is a $\zeta \in C\left([0,1] ; \mathrm{M}_{\ell}\right)$ such that $\zeta(0)=x$ and $\zeta(1)=x^{*}$. Defining

$$
H_{3}(\lambda, t) \stackrel{\text { def }}{=} \mathfrak{z}_{t}(\zeta(\lambda))
$$

for all $\lambda \in[0,1]$ and $t \in \mathbb{R}$, we have the desired homotopy.

We next claim that $\mathbf{p}_{\ell}(\mathbb{R})=\Gamma_{\ell}$. Clearly $q(\mathbb{R}) \subset \Gamma_{\ell}$. By solving the ODE (A.6) backwards in time and using Lemma A.2, we can conclude that $\Gamma_{\ell} \backslash \mathscr{X} \subset q(\mathbb{R})$. Since $q$ is periodic and continuous, $q(\mathbb{R})$ is closed (it is the forward image of a sufficiently large closed interval through a continuous function). Thus $\Gamma_{\ell} \subset$ $\overline{\Gamma_{\ell} \backslash \mathscr{X}} \subset q(\mathbb{R})$. We further claim that $q$ is injective on $\hat{A} \cap\left[0, \mathfrak{T}\left(x^{*}\right)\right)$. Indeed, if $q(t)=q\left(t^{\prime}\right)$ for some distinct $t$ and $t^{\prime}$ in $\hat{A} \cap\left[0, \mathfrak{T}\left(x^{*}\right)\right)$, then by solving (A.6) backwards, we would have that $\mathfrak{z}_{t}\left(x^{*}\right)=\mathfrak{z}_{t^{\prime}}\left(x^{*}\right)$, which is impossible since the 
period of $t \mapsto \mathfrak{z} t\left(x^{*}\right)$ is $\mathfrak{T}\left(x^{*}\right)$. It is also clear that $q\left(t+\mathfrak{T}\left(x^{*}\right)\right)=q(t)$ for all $t \in \mathbb{R}$. We can then calculate that

$$
\begin{aligned}
\tau\left(\mathfrak{T}\left(x^{*}\right)\right) & =\int_{s=0}^{\mathfrak{T}\left(x^{*}\right)} I(q(s))\|\nabla \mathrm{H}(q(s))\|\|\dot{q}(s)\| d s \\
& =\int_{q(\mathbb{R})} J(z)\|\nabla \mathrm{H}(z)\| \mathscr{H}^{1}(d z)=\mathscr{G}_{\ell} .
\end{aligned}
$$

Thus $\tau^{*}\left(t+\mathscr{G}_{\ell}\right)=\tau^{*}(t)+\hat{T}$, so $\mathbf{p}_{\ell}$ is indeed periodic of period $\mathscr{G}_{\ell}$. Furthermore, $\mathbf{p}_{\ell}$ is injective on $\tau\left(\hat{A} \cap\left[0, \mathfrak{T}\left(x^{*}\right)\right)=A_{\ell} \cap \mathcal{I}_{\ell}\right.$.

Proof of Lemma 2.12: First, note that $\mathbf{p}_{\ell}(0)=\mathbf{p}_{\ell}\left(\mathscr{G}_{\ell}\right) \in \mathscr{X}$, so $A_{\ell}$ cannot contain either 0 or $\mathscr{G}_{\ell}$. Second, since $\mathbb{R} \backslash A_{\ell}$ is discrete, we must have that $A \cap \mathcal{I}_{\ell}=$ $\bigcup_{j=0}^{n-1}\left(a_{j}, a_{j+1}\right)$ for some $a_{j}$ 's such that $a_{0}=0$ and $a_{n}=\mathscr{G}_{\ell}$. The differential equations for $\mathbf{p}_{\ell}$ and $\bar{\Theta}$ imply that for each $j \in\{0, \ldots, n\}$, there is a constant $c_{j}$ such that $\bar{\Theta}\left(\mathbf{p}_{\ell}(t)\right)=t+c_{j}$ for all $t \in\left(a_{j}, a_{j+1}\right)$. We then define $\overline{\mathrm{C}}_{\ell}$ by setting $\overline{\mathrm{C}}_{\ell}\left(\mathbf{p}_{\ell}(t)\right)=-c_{j}$ for all $t \in\left(a_{j}, a_{j+1}\right)$. Remark 2.13 ensures that $\overline{\mathrm{C}}_{\ell}$ is welldefined.

Proof of Lemma 2.16: The smoothness of $\wp$ and $\bar{\Theta}$ imply that $\Theta$ is $C^{\infty}$ on $\mathrm{M} \backslash \mathbf{X}$. For any fixed $\mathfrak{x} \in \mathscr{X}, \Theta$ differs from the smooth function $\bar{\Theta}_{\mathfrak{x}}^{\sim} \circ \phi_{\mathfrak{x}} \circ \wp$ by a function that is locally constant on $\wp^{-1}\left(\tilde{\phi}_{\mathfrak{x}}(\square)\right) \backslash \mathbf{X}$. This implies the bounds of the first line of (2.5) on $\wp^{-1}\left(\tilde{\phi}_{\mathfrak{x}}(\square)\right)$. The smoothness in $\mathrm{M} \backslash \mathbf{X}$ implies the bounds on the first line of (2.5) on $\mathrm{M} \backslash \bigcup_{\mathfrak{x} \in \mathscr{X}} \wp^{-1}\left(\tilde{\phi}_{\mathfrak{x}}(\square)\right)$.

Fix now $\mathfrak{x} \in \mathscr{X}$. By the explicit formula for $\tilde{\Theta}_{\mathfrak{x}}$, there is a constant $K>0$ such that

$$
\left|\tilde{\Theta}_{\mathfrak{x}}(x)\right| \leq K\|x\|_{\infty}^{2}, \quad\left\|\nabla_{E} \tilde{\Theta}_{\mathfrak{x}}(x)\right\| \leq K\|x\|_{\infty}, \quad \text { and } \quad\left|D^{2} \tilde{\Theta}_{\mathfrak{x}}(x)\right| \leq K,
$$

for all $x \in \square$. This gives the stated bounds on $\tilde{\Theta}_{\mathfrak{x}}$ and $\nabla_{E} \tilde{\Theta}_{\mathfrak{x}}$ on $\square$.

It only remains to prove the last bound of (2.5). Define first

$$
S \stackrel{\text { def }}{=}\left\{x \in \mathrm{M}:|\mathrm{H}(x)| \leq \frac{\varpi^{2}}{2} \frac{\mho_{-}}{\mho_{+}}\right\} .
$$

By smoothness, there clearly is a $K>0$ such that (2.5) holds on $\mathrm{M} \backslash S$. Consider next the set $A \stackrel{\text { deff }}{=} \overline{\backslash \bigcup_{\mathfrak{x} \in \mathscr{X}} \mathbf{B}_{\mathfrak{x}}}$. From (3.6), we know that $A \cap \mathbf{X}=\varnothing$. Since $A$ is compact and does not intersect $\mathbf{X}$, there is a uniform bound on $\left\{\mathbf{V}\left(\wp_{t}^{*}(x)\right): t \in\right.$ $[0,1), x \in A\}$. Writing

$$
\begin{aligned}
(\bar{\nabla} \mathrm{H}, \nabla \Theta)(x)-\mathrm{J}(x)\|\nabla \mathrm{H}(x)\|^{2}= & \{(\bar{\nabla} \mathrm{H}, \nabla \Theta)(x)-(\bar{\nabla} \mathrm{H}, \nabla \Theta)(\wp(x))\} \\
& -\left\{J(x)\|\nabla \mathrm{H}(x)\|^{2}-\beth(\wp(x))\|\nabla \mathrm{H}(\wp(x))\|^{2}\right\},
\end{aligned}
$$


we get the last bound of (2.5) on $A$. Finally, fixing $\mathfrak{x} \in \mathscr{X}$, we have that

$$
\begin{aligned}
(\bar{\nabla} \mathrm{H}, & \nabla \Theta)\left(\tilde{\phi}_{\mathfrak{x}}(x)\right)-\beth\left(\tilde{\phi}_{\mathfrak{x}}(x)\right)\left\|\nabla \mathrm{H}\left(\tilde{\phi}_{\mathfrak{x}}(x)\right)\right\|^{2} \\
= & \frac{1}{\tilde{\mathrm{B}}_{\mathfrak{x}}(x)}\left\{x_{1} \frac{\partial \tilde{\Theta}_{\mathfrak{x}}}{\partial x_{1}}\left(x_{1}, x_{2}\right)-x_{2} \frac{\partial \tilde{\Theta}_{\mathfrak{x}}}{\partial x_{1}}\left(x_{1}, x_{2}\right)-\tilde{F}_{\mathfrak{x}}\left(x_{1}, x_{2}\right)\right\} \\
= & \frac{1}{\tilde{\mathrm{B}}_{\mathfrak{x}}(x)} x_{1}\left\{\frac{\partial \tilde{\Theta}_{\mathfrak{x}}}{\partial x_{1}}\left(x_{1}, x_{2}\right)-\frac{\partial \tilde{\Theta}_{\mathfrak{x}}}{\partial x_{1}}\left(x_{1}, 0\right)\right\} \\
& \quad-\frac{1}{\tilde{\mathrm{B}}_{\mathfrak{x}}(x)} x_{2} \frac{\partial \tilde{\Theta}_{\mathfrak{x}}}{\partial x_{1}}\left(x_{1}, x_{2}\right)-\frac{1}{\tilde{\mathrm{B}}_{\mathfrak{x}}(x)}\left\{\tilde{F}_{\mathfrak{x}}\left(x_{1}, x_{2}\right)-\tilde{F}_{\mathfrak{x}}\left(x_{1}, 0\right)\right\}
\end{aligned}
$$

for $x=\left(x_{1}, x_{2}\right) \in \square$, and using the above bounds on $\nabla_{E} \tilde{\Theta}_{\mathfrak{x}}$ and $D^{2} \tilde{\Theta}_{\mathfrak{x}}$, we can get the last bound of $(2.5)$ on $\tilde{\phi}_{\mathfrak{x}}\left(\mathcal{C}_{\mathfrak{x}, L} \cup \mathcal{C}_{\mathfrak{x}, R}\right)$. Similar calculations on $\tilde{\phi}_{\mathfrak{x}}\left(\mathscr{X}_{\mathfrak{x}, U} \cup \mathcal{C}_{\mathfrak{x}, D}\right)$ complete the proof of (2.5) on $\mathbf{B}_{\mathfrak{x}}$.

\section{Appendix B: Khasminskii PDE}

We copy some of the arguments of [9]. Essentially, we want to construct the $\Psi_{\ell}$ 's of Proposition 3.1 by Neumann-Dirichlet operators on $\boldsymbol{\Gamma}$. Note that

$$
\Gamma=\bigcup_{\ell \in \Lambda_{P}} \Gamma_{\ell}=\bigcup_{\ell \in \Lambda_{W}} \Gamma_{\ell}
$$

Recall that by Remark 2.13, there is a correspondence between $\mathcal{I}_{\ell}$ of (2.4) and $\Gamma_{\ell}$. Define the Cartesian products

$$
\mathbf{L}^{2}(+) \stackrel{\text { def }}{=} \times_{\ell \in \Lambda_{P}} L^{2}\left(\mathcal{I}_{\ell}\right) \quad \text { and } \quad \mathbf{L}^{2}(+) \stackrel{\text { def }}{=} \times_{\ell \in \Lambda_{W}} L^{2}\left(\mathcal{I}_{\ell}\right)
$$

i.e., elements $\xi^{+}$and $\xi^{\prime}$ of $\mathbf{L}^{2}(+)$ and $\mathbf{L}^{2}(-)$ are, respectively, vectors $\left(\xi_{\ell}^{+}: \ell \in\right.$ $\left.\Lambda_{P}\right)$ and $\left(\xi_{\ell}^{-}: \ell \in \Lambda_{W}\right)$ of functions. Let $B(\Gamma)$ be the collection of bounded and measurable functions on $\boldsymbol{\Gamma}$. Each fixed $f \in B(\boldsymbol{\Gamma})$ defines $\xi_{+}^{f} \in \mathbf{L}^{2}(+)$ and $\xi_{-}^{f} \in \mathbf{L}^{2}(-)$ by the formulae

$$
\begin{array}{ll}
\left(\xi_{+}^{f}\right)_{\ell}(\theta) \stackrel{\text { def }}{=} f\left(\mathbf{p}_{\ell}(\theta)\right) & \text { for } \ell \in \Lambda_{P} \text { and } \theta \in \mathcal{I}_{\ell}, \\
\left(\xi_{-}^{f}\right)_{\ell}(\theta) \stackrel{\text { def }}{=} f\left(\mathbf{p}_{\ell}(\theta)\right) & \text { for } \ell \in \Lambda_{W} \text { and } \theta \in \mathcal{I}_{\ell} ;
\end{array}
$$

this allows us to construct elements of $\mathbf{L}^{2}(+)$ and $\mathbf{L}^{2}(-)$ from elements of $B(\boldsymbol{\Gamma})$. By reversing these formulae, we can conversely recover functions on $\Gamma \backslash \mathscr{X}$ from elements of either $\mathbf{L}^{2}(+)$ or $\mathbf{L}^{2}(-)$ (we need to be careful at elements of $\mathscr{X}$ since $\mathbf{p}_{\ell}^{-1}(\{\mathfrak{x}\}) \cap \mathcal{I}_{\ell}$ may contain more than one element; one of the advantages of $L^{2}$ functions is that they are defined only up to sets of measure zero). 
Let's next define some transition maps. For $\xi^{+} \in \mathbf{L}^{2}(+)$ and $\xi^{-} \in \mathbf{L}^{2}(-)$, define $\boldsymbol{\iota}_{-,+}\left(\xi^{+}\right) \in \mathbf{L}^{2}(-)$ and $\boldsymbol{\iota}_{+,-}\left(\xi^{-}\right) \in \mathbf{L}^{2}(+)$ by setting

$$
\begin{aligned}
& \left(\boldsymbol{\iota}_{-,+}\left(\xi^{+}\right)\right)_{\ell}(\theta) \stackrel{\text { def }}{=} \sum_{\ell^{\prime} \in \Lambda_{P}} \xi_{\ell^{\prime}}^{+}\left(\iota_{\ell^{\prime}, \ell}(\theta)\right) \chi_{\Theta_{\ell}\left(\gamma_{\ell, \ell^{\prime}}\right)}(\theta), \quad \ell \in \Lambda_{W}, \theta \in \mathcal{I}_{\ell}, \\
& \left(\boldsymbol{\iota}_{+,-}\left(\xi^{-}\right)\right)_{\ell}(\theta) \stackrel{\text { def }}{=} \sum_{\ell^{\prime} \in \Lambda_{W}} \xi_{\ell^{\prime}}^{+}\left(\iota_{\ell^{\prime}, \ell}(\theta)\right) \chi_{\Theta_{\ell}\left(\gamma_{\ell, \ell^{\prime}}\right)}(\theta), \quad \ell \in \Lambda_{P}, \theta \in \mathcal{I}_{\ell} .
\end{aligned}
$$

Then $\boldsymbol{\iota}_{-,+}$and $\boldsymbol{\iota}_{+,-}$are inverses of each other, and if $f \in B(\boldsymbol{\Gamma})$ and $\xi_{+}^{f}$ and $\xi_{-}^{f}$ are as in (B.1), then $\boldsymbol{\iota}_{-,+} \xi_{+}^{f}=\xi_{-}^{f}$.

Let's next define smooth functions. For $\ell \in \Lambda, k \in \mathbb{Z}$, and $\xi \in L^{2}\left(\mathcal{I}_{\ell}\right)$, define

$$
\mathrm{c} \ell, k[\xi] \stackrel{\text { def }}{=} \frac{1}{\mathscr{G}_{\ell}} \int_{\theta \in \mathcal{I}_{\ell}} \xi(\theta) e^{-\frac{2 \pi k \theta \sqrt{-1}}{\mathscr{G}_{\ell}}} d \theta
$$

For each $\ell \in \Lambda$, define

$C_{p}^{\infty}\left(\mathcal{I}_{\ell}\right) \stackrel{\text { def }}{=}\left\{\xi \in L^{2}\left(\mathcal{I}_{\ell}\right): \sum_{k \in \mathbb{Z}}\left|c_{\ell, k}[\xi]\right|^{2}\left(1+|k|^{n}\right)<\infty\right.$ for all positive integers $\left.n\right\} ;$ any element of $C_{p}^{\infty}\left(\mathcal{I}_{\ell}\right)$ defines a smooth and $\mathscr{G}_{\ell}$-periodic function on $\mathbb{R}$. Define

$$
\mathbf{C}_{p}^{\infty}(+) \stackrel{\text { def }}{=} \times_{\ell \in \Lambda_{P}} C_{p}^{\infty}\left(\mathcal{I}_{\ell}\right) \quad \text { and } \quad \mathbf{C}_{p}^{\infty}(-) \stackrel{\text { def }}{=} \times_{\ell \in \Lambda_{P}} C_{p}^{\infty}\left(\mathcal{I}_{\ell}\right) .
$$

Insert word "define" between "finally" and "classes"?
Let's finally classes of admissible functions. Set

$$
\mathfrak{A}(+) \stackrel{\text { def }}{=} \mathbf{C}_{p}^{\infty}(+) \cap \boldsymbol{\iota}_{+,-} \mathbf{C}_{p}^{\infty}(-) \quad \text { and } \quad \mathfrak{A}(-) \stackrel{\text { def }}{=} \mathbf{C}_{p}^{\infty}(-) \cap \boldsymbol{\iota}_{-,+} \mathbf{C}_{p}^{\infty}(+) .
$$

Fix now $\ell \in \Lambda$ and $\xi \in C_{p}^{\infty}\left(\mathcal{I}_{\ell}\right)$. Suppose that we have boundary data $\xi \circ \Theta_{\ell}$ on $\Gamma_{\ell}$. We can then solve the PDE (3.2) subject to the periodicity requirement of (3.1), the decay requirement of (3.3), and Dirichlet data $\xi$ at $h=0$. We can then read off the Neumann data. For $\ell \in \Lambda$ and $k \in \mathbb{Z}$, set

$$
\lambda_{\ell, k} \stackrel{\text { def }}{=} \begin{cases}\sqrt{\frac{2 \pi}{\mathscr{G}_{\ell}}} \sqrt{|k|}(1-\sqrt{-1}) & \text { if } k>0 \\ 0 & \text { if } k=0 \\ \sqrt{\frac{2 \pi}{\mathscr{G}_{\ell}}} \sqrt{|k|}(1+\sqrt{-1}) & \text { if } k<0 .\end{cases}
$$

Also, define $\mathrm{s} \ell \stackrel{\text { def }}{=} 1$ for $\ell \in \Lambda_{P}$ and $\mathrm{s} \ell \stackrel{\text { def }}{=}-1$ for $\ell \in \Lambda_{W}$. Set

$$
\mathfrak{b}_{\ell}[\xi](\theta) \stackrel{\text { def }}{=}-\mathrm{s}_{\ell} \sum_{k \in \mathbb{Z} \backslash\{0\}} \lambda_{\ell, k} \exp \left[\frac{2 \pi k \theta \sqrt{-1}}{\mathscr{G}_{\ell}}\right] \mathfrak{c} \ell, k[\xi], \quad \theta \in \mathcal{I}_{\ell} .
$$

Define then $\mathfrak{b}_{+}: \mathbf{C}_{p}^{\infty}(+) \rightarrow \mathbf{L}^{2}(+)$ and $\mathfrak{b}_{-}: \mathbf{C}_{p}^{\infty}(-) \rightarrow \mathbf{L}^{2}(-)$ by $\left(\mathfrak{b}_{+} \xi\right)_{\ell} \stackrel{\text { def }}{=} \mathfrak{b}_{\ell} \xi_{\ell}$ for $\ell \in \Lambda_{P}$ and $\xi \in \mathbf{C}_{p}^{\infty}(+)$ and $\left(\mathfrak{b}_{-} \xi\right)_{\ell} \stackrel{\text { def }}{=} \mathfrak{b}_{\ell} \xi_{\ell}$ for $\ell \in \Lambda_{P}$ and $\xi \in \mathbf{C}_{p}^{\infty}(-)$. 
Define now $\mathbf{P}_{+} \in \mathbf{L}^{2}(+)$ as $\left(\mathbf{P}_{+}\right)_{\ell} \equiv \mathbf{v}_{\ell}$ for each $\ell \in \Lambda_{P}$ and define $\mathbf{P}_{-} \in \mathbf{L}^{2}(-)$ as $\left(\mathbf{P}_{-}\right)_{\ell} \equiv \mathbf{v}_{\ell}$ for each $\ell \in \Lambda_{W}$. Fix $\xi \in \mathfrak{A}(+)$ and assume that it comes from Dirichlet data $f$ on $\Gamma$ (as in (B.1)). The continuity conditions (3.5) require that

$$
\boldsymbol{\iota}_{+,-}\left(\mathfrak{b}_{-} \boldsymbol{\iota}_{-,+}(\xi)+\mathbf{P}_{-}\right)=\mathfrak{b}_{+} \xi+\mathbf{P}_{+} ;
$$

i.e., we would like to solve

$$
\hat{\mathbf{K}} \xi=\hat{\mathbf{P}}
$$

where $\hat{\mathbf{K}} \xi \stackrel{\text { def }}{=} \boldsymbol{\iota}_{+,-}\left(\mathfrak{b}_{-} \boldsymbol{\iota}_{-,+}(\xi)\right)-\mathfrak{b}_{+} \xi$ for all $\xi \in \mathfrak{A}(+)$ and where $\hat{\mathbf{P}} \stackrel{\text { def }}{=} \mathbf{P}_{+}-\boldsymbol{\iota}_{+,-} \mathbf{P}_{-}$.

As in [9], we cannot necessarily find $\xi \in \mathfrak{A}(+)$ satisfying (B.2); we can, however, do so in a larger space. Define first an inner product $\langle\cdot, \cdot\rangle_{\mathbf{L}^{2}(+)}$ on $\mathbf{L}^{2}(+)$ in the usual way, by defining

$$
\left\langle\xi, \xi^{\prime}\right\rangle_{\mathbf{L}^{2}(+)} \stackrel{\text { def }}{=} \sum_{\ell \in \Lambda_{P}}\left\langle\xi_{\ell}, \xi_{\ell}^{\prime}\right\rangle_{L^{2}\left(\mathcal{I}_{\ell}\right)}
$$

for $\xi$ and $\xi$ in $\mathbf{L}^{2}(+)$. Also, for $\xi \in \mathbf{L}^{2}(+)$, define $\bar{\xi} \in \mathbf{L}^{2}(+) \times \mathbf{L}^{2}(-)$ by setting $\bar{\xi}_{\ell} \stackrel{\text { def }}{=} \xi_{\ell}$ for $\ell \in \Lambda_{P}$ and by setting $\bar{\xi}_{\ell} \stackrel{\text { def }}{=}\left(\boldsymbol{\iota}_{-,+} \xi\right)_{\ell}$ for $\ell \in \Lambda_{W}$; this extends elements of $\mathbf{L}^{2}(+)$ to elements of $\mathbf{L}^{2}(-)$ in a way that respects $\boldsymbol{\iota}_{-,+}$. We then have the following:

Lemma B.1 For $\xi \in \mathfrak{A}(+)$,

$$
\langle\mathbf{K}[\xi], \xi\rangle_{\mathbf{L}^{2}(+)}=\left.\left.\frac{1}{\sqrt{2}} \sum_{\substack{\ell \in \Lambda \\ k \in \mathbb{Z} \backslash\{0\}}}\left|\lambda_{\ell, k}\right|\right|_{c_{\ell, k}}\left[\bar{\xi}_{\ell}\right]\right|^{2}
$$

For $\xi$ and $\xi^{\prime}$ in $\mathfrak{A}(+)$,

$$
\left\langle\mathbf{K}[\xi], \xi^{\prime}\right\rangle_{\mathbf{L}^{2}(+)} \leq\left\{\sum_{\substack{\ell \in \Lambda \\ k \in \mathbb{Z} \backslash\{0\}}}\left|c_{\ell, k}\left[\bar{\xi}_{\ell}\right]\right|^{2}\left|\lambda_{\ell, k}\right|\right\}^{\frac{1}{2}}\left\{\sum_{\substack{\ell \in \Lambda \\ k \in \mathbb{Z} \backslash\{0\}}}\left|c_{\ell, k}\left[\bar{\xi}_{\ell}^{\prime}\right]\right|^{2}\left|\lambda_{\ell, k}\right|\right\}^{\frac{1}{2}} .
$$

PROOF: The proof is essentially the same as that of lemmas 9.2 and 9.3 of [9]. We leave the details to the reader.

Let's finally start to set things up. For all $\xi$ and $\xi^{\prime}$ in $\mathfrak{A}(+)$, define

$$
\left\langle\xi, \xi^{\prime}\right\rangle_{\mathbf{H}} \stackrel{\text { def }}{=} \sum_{\substack{\ell \in \Lambda \\ k \in \mathbb{Z} \backslash\{0\}}}\left|\lambda_{\ell, k}\right|_{\mathfrak{c} \ell, k}\left[\bar{\xi}_{\ell}\right]_{\mathrm{c}_{\ell, k}}^{*}\left[\bar{\xi}_{\ell}^{\prime}\right]+\left\langle\xi, \xi^{\prime}\right\rangle_{\mathbf{L}^{2}(+)}
$$

where $x^{*}$ is the complex conjugate of $x$ for any $x \in \mathbb{C}$. Define also

$$
\|\xi\|_{\mathbf{H}} \stackrel{\text { def }}{=} \sqrt{\langle\xi, \xi\rangle_{\mathbf{H}}}=\left\{\left.\left.\sum_{\substack{\ell \in \Lambda \\ k \in \mathbb{Z} \backslash\{0\}}}\left|\lambda_{\ell, k}\right|\right|_{c_{\ell, k}}\left[\bar{\xi}_{\ell}\right]\right|^{2}+\|\xi\|_{L^{2}(+)}^{2}\right\}^{\frac{1}{2}}
$$

for $\xi \in \mathfrak{A}(+)$. We note that thus $\mathfrak{A}(+)$ is a normed linear space with norm $\|\cdot\|_{\mathbf{H}}$ and with inner product $\langle\cdot, \cdot\rangle_{\mathbf{H}}$. Let $\mathbf{H}$ be the closure of $\mathfrak{A}(+)$ with respect to $\|\cdot\|_{\mathbf{H}}$ 
(see [8, theorem 7.4]). We also define the bilinear mapping $\mathbf{B}: \mathfrak{A}(+) \times \mathfrak{A}(+) \rightarrow \mathbb{R}$ by setting

$$
\mathbf{B}_{K}\left[\xi, \xi^{\prime}\right] \stackrel{\text { def }}{=}\left\langle\mathbf{K}[\xi], \xi^{\prime}\right\rangle_{\mathbf{L}^{2}(+)}
$$

for all $\xi$ and $\xi^{\prime}$ in $\mathfrak{A}(+)$.

LEMMA B.2 The space $\mathbf{H}$ is a Hilbert space that is compactly embedded in $\mathbf{L}^{2}(+)$. Furthermore, there is a unique extension of $\mathbf{B}_{K}$ to a bilinear bounded mapping on $\mathbf{H} \times \mathbf{H}$.

PROOF: The proof is the same as the proof of lemma 9.4 in [9].

Now let $\mathbf{1} \in \mathbf{L}^{2}(+)$ be defined as

$$
\mathbf{1}_{\ell} \equiv 1, \quad \ell \in \Lambda_{P}
$$

Note that

$$
\langle\hat{\mathbf{P}}, \mathbf{1}\rangle_{\mathbf{L}^{2}(+)}=\sum_{\ell \in \Lambda_{P}} \mathscr{G}_{\ell} \mathbf{v}_{\ell}-\sum_{\ell \in \Lambda_{W}} \mathscr{G}_{\ell} \mathbf{v}_{\ell}
$$

thus the gluing conditions of (2.2) mean exactly that $\langle\hat{\mathbf{P}}, \mathbf{1}\rangle_{\mathbf{L}^{2}(+)}=0$.

Note now the following:

LEMMA B.3 If $\xi \in \mathbf{L}^{2}(+)$ is such that $c_{\ell, k}\left[\bar{\xi}_{\ell}\right]=0$ for all $\ell \in \Lambda$ and $k \in \mathbb{Z} \backslash\{0\}$, then $\xi$ is in fact constant; i.e., there is a $c \in \mathbb{R}$ such that $\bar{\xi}_{\ell} \equiv c$ for all $\ell \in \Lambda$.

PROOF: Under the assumptions, for each $\ell \in \Lambda$ there is a $c_{\ell} \in \mathbb{R}$ such that $\bar{\xi}_{\ell} \equiv c_{\ell}$. Define $f(x) \stackrel{\text { def }}{=} \sum_{\ell \in \Lambda} c_{\ell} \chi_{\mathrm{M}_{\ell}}(x)$ for all $x \in \mathrm{M} \backslash \Gamma$. Since $\xi \in \mathbf{H}$, the function

$$
\hat{f}(x) \stackrel{\text { def }}{=} \lim _{\substack{x^{\prime} \rightarrow \\ x^{\prime} \in \mathbb{M} \backslash \Gamma}} f\left(x^{\prime}\right)
$$

exists for all $x \in \mathrm{M} \backslash \mathscr{X}$; in particular, the limit exists on $\Gamma \backslash \mathscr{X}^{3}$ Thus $\hat{f}$ is a continuous function. Since $\mathrm{M}$ is a smooth, connected manifold, it is arcwise connected. Removing $\mathscr{X}$, we can verify that $\mathrm{M} \backslash \mathscr{X}$ is also arcwise connected. Thus $\hat{f}$ is a continuous and arcwise-constant function on an arcwise connected space. Hence $\hat{f}$ is in fact constant, so all of the $c_{\ell}$ 's coincide.

We now have the following:

Proposition B.4 If $F \in \mathbf{L}^{2}(+)$ is such that $\langle F, \mathbf{1}\rangle_{\mathbf{L}^{2}(+)}=0$, then there is a $\xi \in \mathbf{H}$ such that

$$
\mathbf{B}_{K}[\xi, \zeta]=\langle F, \zeta\rangle_{\mathbf{L}^{2}(+)}
$$

\footnotetext{
${ }^{3}$ Approximate by elements of $\mathfrak{A}(+)$ and integrate against smooth (e.g., mollifier) functions; we get that the limit of (B.3) exists at $\mathscr{H}^{1}$-almost every point of $\Gamma \backslash \mathscr{X}$; since the functions are in fact constants, the limit exists at all points of $\Gamma \backslash \mathscr{X}$.
} 
for all $\zeta \in \mathbf{H}$ and such that

$$
\mathbf{B}_{K}[\xi, \xi] \leq\left(\frac{\overline{\mathscr{G}}}{\pi}\right)^{\frac{1}{2}}\|F\|_{\mathbf{L}^{2}(+)}^{2} .
$$

PROOF: The proof is similar to the proof of lemma 9.5 of [9] (see also [3, lemma 6.2.4]); due to the complexity of the argument, we shall give the full proof. For each $f \in \mathbf{L}^{2}(+)$, define

$$
L_{f}[\xi] \stackrel{\text { def }}{=}\langle f, \xi\rangle_{\mathbf{L}^{2}(+)}
$$

for all $\xi \in \mathbf{H}$. By the compact embedding result of Lemma B.2, $L_{f}$ is a bounded linear operator on $\mathbf{H}$. Define the bounded linear operator

$$
\overline{\mathbf{B}}[\xi, \zeta] \stackrel{\text { def }}{=} \mathbf{B}_{K}[\xi, \zeta]+\frac{1}{\sqrt{2}}\langle\xi, \zeta\rangle_{\mathbf{L}^{2}(+)}
$$

for all $\xi$ and $\zeta$ in $\mathbf{H}$. Thus by Lemma B.1,

$$
\begin{gathered}
\overline{\mathbf{B}}[\xi, \xi]=\frac{1}{\sqrt{2}}\|\xi\|_{\mathbf{H}}^{2}, \quad \xi \in \mathbf{H}, \\
|\overline{\mathbf{B}}[\xi, \zeta]| \leq\left(1+\frac{1}{\sqrt{2}}\right)\|\xi\|_{\mathbf{H}}\|\zeta\|_{\mathbf{H}}, \quad \xi, \zeta \in \mathbf{H} .
\end{gathered}
$$

By the Lax-Milgram lemma, for each $f \in \mathbf{L}^{2}(+)$, we can find a unique $\mathbf{A}[f] \in \mathbf{H}$ such that

$$
\overline{\mathbf{B}}[\mathbf{A}[f], \zeta]=L_{f}[\zeta]
$$

for all $\zeta \in \mathbf{H}$. By coercivity, the boundedness of $L_{f}$, and the compact embedding result of Lemma B.2, $\mathbf{A}$ is a compact linear operator from $\mathbf{L}^{2}(+)$ into itself. We thus want to find $a \xi \in \mathbf{L}^{2}(+)$ such that

$$
\xi=\mathbf{A}\left[F+\frac{1}{\sqrt{2}} \xi\right]=\mathbf{A}[F]+\frac{1}{\sqrt{2}} \mathbf{A}[\xi]
$$

in $\mathbf{L}^{2}(+)$. By the Fredholm alternative, we can do this if and only if $\mathbf{A}[F]$ is orthogonal in $\mathbf{L}^{2}(+)$ to $\left\{\zeta \in \mathbf{L}^{2}(+): \zeta=(1 / \sqrt{2}) \mathbf{A}^{*}[\zeta]\right\}$, where $\mathbf{A}^{*}$ is the $\mathbf{L}^{2}(+)$ adjoint of A. Equivalently, we can solve (B.4) if and only if $F$ is orthogonal in $\mathbf{L}^{2}(+)$ to the subspace

$$
\begin{aligned}
M & \stackrel{\text { def }}{=}\left\{\mathbf{A}^{*}[\zeta]: \zeta \in \mathbf{L}^{2}(+) \text { and } \zeta=\frac{1}{\sqrt{2}} \mathbf{A}^{*}[\zeta]\right\} \\
& =\left\{\zeta \in \mathbf{L}^{2}(+): \zeta=\frac{1}{\sqrt{2}} \mathbf{A}^{*}[\zeta]\right\}
\end{aligned}
$$

of $\mathbf{L}^{2}(+)$. We want to show that $M$ consists only of constants; by Lemma B.1, we want to show that $\mathbf{B}[\zeta, \zeta]=0$ for $\zeta \in M$. It is easy to see that for all $\xi$ and $\zeta^{\prime}$ 
in $\mathfrak{A}(+), \overline{\mathbf{B}}\left[\xi, A^{*}\left[\zeta^{\prime}\right]\right]=L_{\zeta^{\prime}}[\xi]$. By coercivity and compact embedding, we can show that $\mathbf{A}^{*} \mathbf{L}^{2}(+) \subset \mathbf{H}$; thus $M \subset \mathbf{H}$. For all $u \in \mathbf{H}$,

$$
\begin{aligned}
\mathbf{B}_{K}[\mathbf{A}[u], \zeta] & =\overline{\mathbf{B}}[\mathbf{A}[u], \zeta]-\frac{1}{\sqrt{2}}\langle\mathbf{A}[u], \zeta\rangle_{\mathbf{L}^{2}(+)} \\
& =\langle u, \zeta\rangle_{\mathbf{L}^{2}(+)}-\frac{1}{\sqrt{2}}\langle\mathbf{A}[u], \zeta\rangle_{\mathbf{L}^{2}(+)} \\
& =\langle u, \zeta\rangle_{\mathbf{L}^{2}(+)}-\frac{1}{\sqrt{2}}\left\langle u, \mathbf{A}^{*}[\zeta]\right\rangle_{\mathbf{L}^{2}(+)}=0 .
\end{aligned}
$$

We would like to take $u \in \mathbf{L}^{2}(+)$ such that $\mathbf{A}[u]=\zeta$, but our above arguments don't immediately imply that $\zeta$ is in the range of $\mathbf{A}$. Thus, we shall approximate. Let $\left(\zeta_{n} ; n \in \mathbb{N}\right)$ be a sequence in $\mathfrak{A}(+)$ such that $\lim _{n} \succ \infty\left\|\zeta_{n}-\zeta\right\|_{\mathbf{H}}=0$, and define $u_{n} \stackrel{\text { def }}{=} \mathbf{K}\left[\zeta_{n}\right]+(1 / \sqrt{2}) \zeta_{n}$ ( since the $\zeta_{n}$ 's are in $\mathfrak{A}(+)$, the $u_{n}$ 's are well-defined; we can also check that $\mathbf{A}\left[u_{n}\right]=\zeta_{n}$ for all $n \in \mathbb{N}$ ). Then

$$
\lim _{n \rightarrow \infty}\left\|\mathbf{A}\left[u_{n}\right]-\zeta\right\|_{\mathbf{H}}=\lim _{n \rightarrow \infty}\left\|\zeta_{n}-\zeta\right\|_{\mathbf{H}}=0,
$$

so since $\mathbf{B}_{K}$ is continuous in the $\mathbf{H}$-norm, we have that $\mathbf{B}_{K}[\zeta, \zeta]=0$, so by Lemma B.3, $\zeta$ is constant, as we wanted to show (use Lemma B.1). Thus $M$ consists of constant functions, so we can solve (B.4) if and only if $F$ is orthogonal in $\mathbf{L}^{2}(+)$ to the constant functions, which is exactly the stated requirement.

Define $\xi^{\prime} \stackrel{\text { def }}{=} \xi-\langle\xi, \mathbf{1}\rangle_{\mathbf{L}^{2}(+)} \mathbf{1}$. Since $\langle F, \mathbf{1}\rangle_{\mathbf{L}^{2}(+)}=0$, we have that

$$
\begin{aligned}
\left.\left.\frac{1}{\sqrt{2}} \sum_{\substack{\ell \in \Lambda_{P} \\
k \in \mathbb{Z}\{0\}}}\left|\lambda_{\ell, k}\right|\right|_{c_{\ell, k}}\left[\xi_{\ell}\right]\right|^{2} & =\mathbf{B}_{K}[\xi, \xi] \\
& =\langle F, \xi\rangle_{\mathbf{L}^{2}(+)} \\
& =\left\langle F, \xi^{\prime}\right\rangle_{\mathbf{L}^{2}(+)} \\
& \leq\|F\|_{\mathbf{L}^{2}(+)}\left\|\xi^{\prime}\right\|_{\mathbf{L}^{2}(+)} \\
& \leq\|F\|_{\mathbf{L}^{2}(+)}\left\{\left.\left.\sum_{\substack{\ell \in \Lambda \\
k \in \mathbb{Z}}}\right|_{c_{\ell, k}}\left[\bar{\xi}_{\ell}^{\prime}\right]\right|^{2}\right\}^{\frac{1}{2}} .
\end{aligned}
$$

Clearly $\mathrm{c} \ell, k_{, k}\left[\bar{\xi}_{\ell}^{\prime}\right]=\mathrm{c} \ell, k_{\ell}\left[\bar{\xi}_{\ell}\right]$ for all $\ell \in \Lambda$ and $k \in \mathbb{Z} \backslash\{0\}$. We also claim that ${ } \ell, 0\left[\bar{\xi}_{\ell}^{\prime}\right]=0$ for all $\ell \in \Lambda$. More precisely, we need to show that $\xi^{\prime}$ is orthogonal (in $\mathbf{L}^{2}(+)$ ) to the set

$$
S \stackrel{\text { def }}{=}\left\{\xi \in \mathbf{L}^{2}(+): c_{\ell, k}\left[\bar{\xi}_{\ell}\right]=0 \text { for all } \ell \in \Lambda \text { and } k \in \mathbb{Z} \backslash\{0\}\right\} .
$$

However, Lemma B.3 tells us that

$$
S=\left\{\xi \in \mathbf{L}^{2}(+): \xi=\langle\xi, \mathbf{1}\rangle_{\mathbf{L}^{2}(+)} \mathbf{1}\right\} .
$$

Clearly $\xi^{\prime}$ is orthogonal to $\mathbf{1}$, so we have that ${ }_{\ell} \ell, 0\left[\bar{\xi}_{\ell}^{\prime}\right]=0$ for all $\ell \in \Lambda$, as desired. 
To proceed, we now note that if $\ell \in \Lambda$ and $k \in \mathbb{Z} \backslash\{0\}$,

$$
1=\frac{\left|\lambda_{\ell, k}\right|}{|k|^{1 / 2}} \sqrt{\frac{\mathscr{G}_{\ell}}{4 \pi}} \leq\left|\lambda_{\ell, k}\right| \sqrt{\frac{\overline{\mathscr{G}}}{4 \pi}},
$$

and thus

$$
\begin{aligned}
\left.\left.\frac{1}{\sqrt{2}} \sum_{\substack{\ell \in \Lambda_{P} \\
k \in \mathbb{Z}\{0\}}}\left|\lambda_{\ell, k}\right|\right|_{c_{\ell, k}}\left[\xi_{\ell}\right]\right|^{2}=\mathbf{B}_{K}[\xi, \xi] \leq \\
\qquad\left(\frac{\overline{\mathscr{G}}}{4 \pi}\right)^{\frac{1}{4}}\|F\|_{\mathbf{L}^{2}(+)}\left\{\sum_{\substack{\ell \in \Lambda \\
k \in \mathbb{Z} \backslash\{0\}}}\left|\lambda_{\ell, k} \|_{c_{\ell, k}}\left[\bar{\xi}_{\ell}^{\prime}\right]\right|^{2}\right\}^{\frac{1}{2}},
\end{aligned}
$$

which leads to the stated upper bound.

We can now give the desired proof:

Proof OF PROPOSITION 3.1: We use the same arguments as in the proof of proposition 8.6 of [9]. We now use Proposition B.4 above instead of proposition 9.5 of [9].

Acknowledgment. This work was supported by NSF Grants DMS 0071484 and DMS 0305925, and ONR Grant N00014-01-1-0647.

\section{Bibliography}

[1] Arnol'd, V. I. Mathematical methods of classical mechanics. 2nd ed. Graduate Texts in Mathematics, 60. Springer, New York, 1989.

[2] Boothby, W. M. An introduction to differentiable manifolds and Riemannian geometry. 2nd ed. Pure and Applied Mathematics, 120. Academic, Orlando, Fla., 1986.

[3] Evans, L. C. Partial differential equations. American Mathematical Society, Providence, R.I., 1998.

[4] Freidlin, M.; Weber, M. Random perturbations of nonlinear oscillators. Ann. Probab. 26 (1998), no. 3, 925-967.

[5] Freidlin, M.; Weber, M. A remark on random perturbations of the nonlinear pendulum. Ann. Appl. Probab. 9 (1999), no. 3, 611-628.

[6] Freidlin, M. I.; Wentzell, A. D. Random perturbations of Hamiltonian systems. Mem. Amer. Math. Soc. 109 (1994), no. 523.

[7] Neřshtadt, A. I. Probability phenomena due to separatrix crossing. Chaos 1 (1991), no. 1, 42-48.

[8] Royden, H. L. Real analysis. 3rd ed. Macmillan, New York, 1988.

[9] Sowers, R. B. Stochastic averaging near a homoclinic orbit with multiplicative noise. Stoch. Dyn. 3 (2003), no. 3, 299-391.

[10] Sowers, R. B. Stochastic averaging near homoclinic orbits via singular perturbations: a proof of a result by Freidlin and Weber using ideas of Khasminskii. IUTAM Symposium on Nonlinear Stochastic Dynamics, 83-95. N. S. Namachchivaya and Y. K. Lin, eds. Kluwer, Boston, 2003. 
RICHARD B. SOWERS

University of Illinois at Urbana-Champaign

Department of Mathematics

1409 W Green Street

Urbana, IL 61801

E-mail: r-sowers@math.uiuc.edu

Received September 2003. 